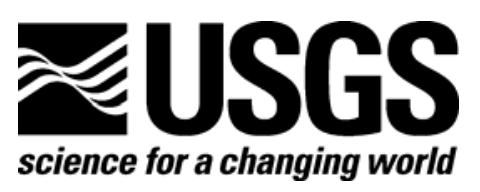

\title{
Surficial Sediment Character of the New York-New Jersey Offshore Continental Shelf Region: a GIS Compilation
}

By S. Jeffress Williams', Matthew A. Arsenault', Lawrence J. Poppe1, Jane A. Reid², Jamey M. Reid ${ }^{1}$, and Chris J. Jenkins ${ }^{3}$

IU.S. Geological Survey, Woods Hole Science Center, 384 Woods Hole Road, Woods Hole, MA 02543

2U.S. Geological Survey, Western Coastal and Marine Geology, Pacific Science Center, 400 Natural Bridges Drive, Santa Cruz, CA 95060

3Institute of Arctic and Alpine Research, University of Colorado at Boulder, 1560 30th Street, Campus Box 450, Boulder, C0 80309-0450

U.S. Geological Survey Open-File Report 2006-1046

U.S. Department of the Interior

U.S. Geological Survey 


\section{U.S. Department of the Interior \\ Dirk Kempthorne, Secretary}

\section{U.S. Geological Survey \\ Mike D. Myers, Director}

U.S. Geological Survey, Reston, Virginia
2007

For product and ordering information:

World Wide Web: http://www.usgs.gov/pubprod

Telephone: 1-888-ASK-USGS

For more information on the USGS - the Federal source for science about the Earth, its natural and living resources, natural hazards, and the environment:

World Wide Web: http://www.usgs.gov

Telephone: 1-888-ASK-USGS

Suggested citation:Suggested citation: This document should be cited as: Williams, S.J., Arsenault, M.A., Poppe, L.J., Reid, J.A., Reid, J.M. and Jenkins, C.J., 2006, Surficial sediment character of the New York-New Jersey offshore Continental Shelf region; a GIS Compilation: U.S. Geological Survey Open-File Report 2006-1046. Online at http://pubs.usgs.gov/of/2006/1046

Any use of trade, product, or firm names is for descriptive purposes only and does not imply endorsement by the U.S. Government.

Although this report is in the public domain, permission must be secured from the individual copyright owners to reproduce any copyrighted material contained within this report. 


\section{Contents}

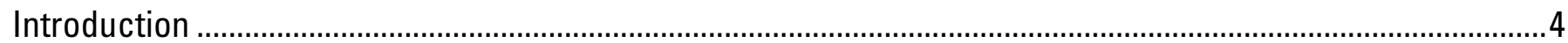

Overview.

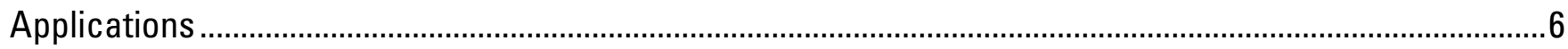

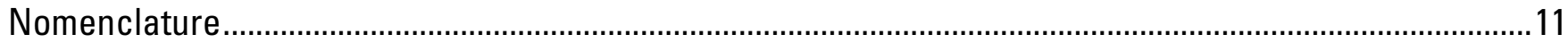

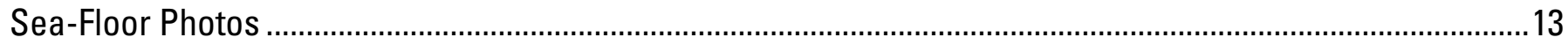

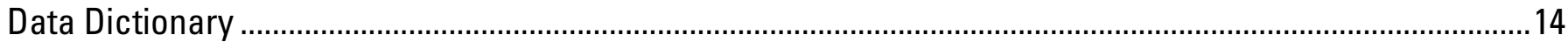

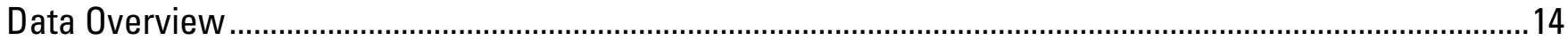

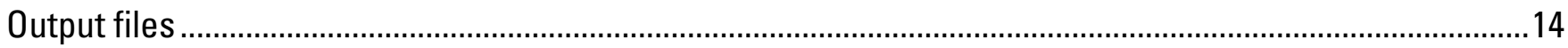

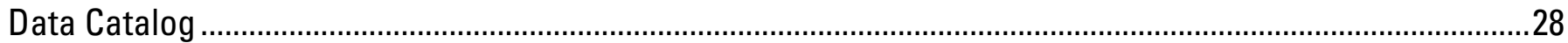

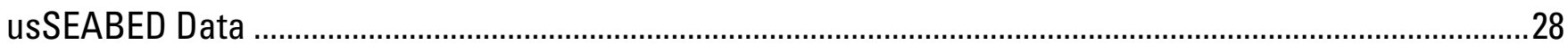

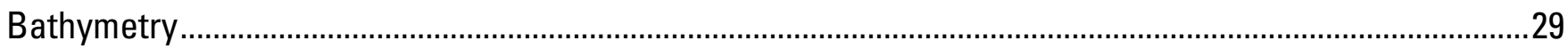

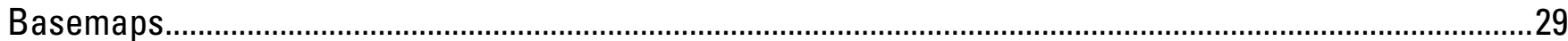

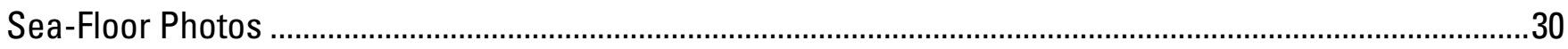

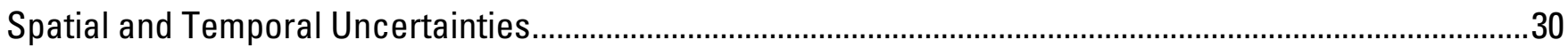

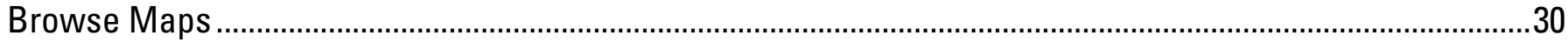

Overview.

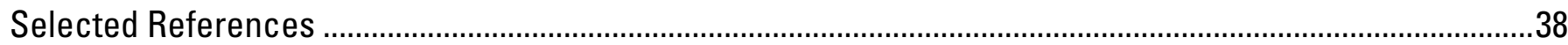

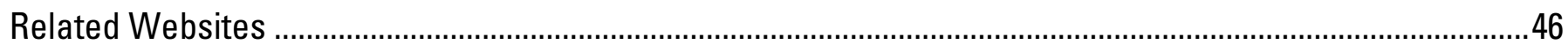

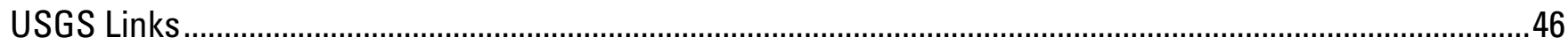

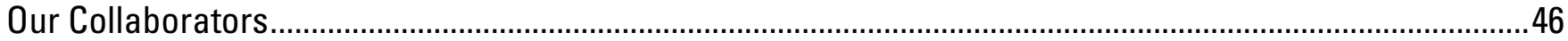

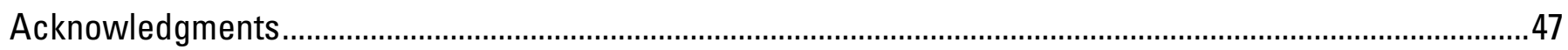

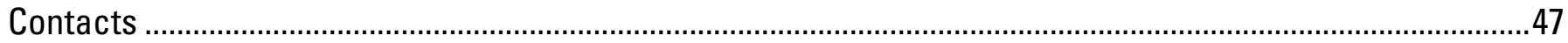

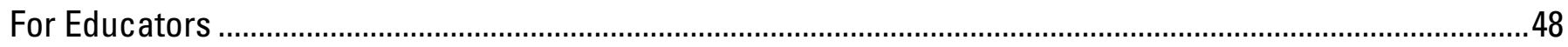

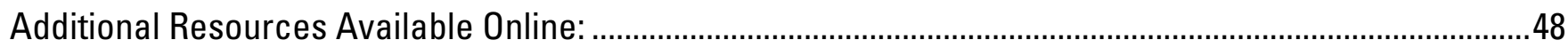

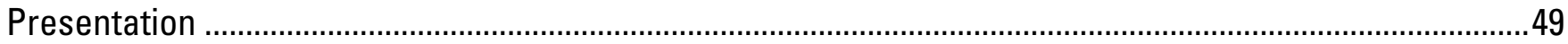

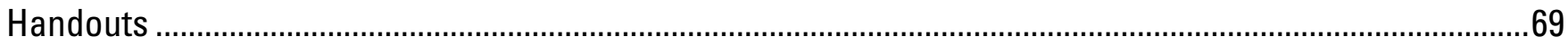

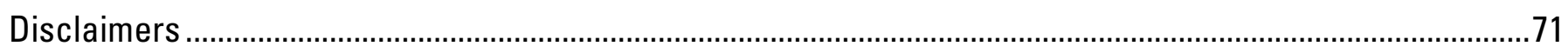

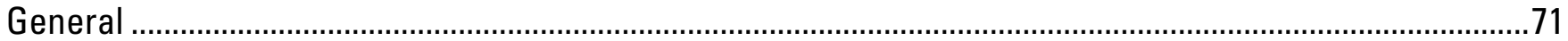

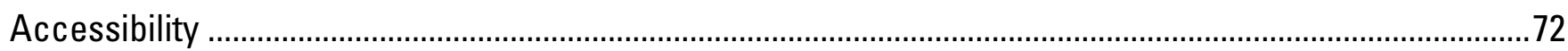

README

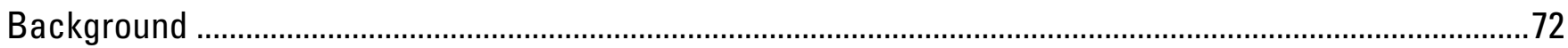

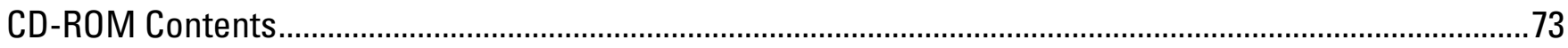

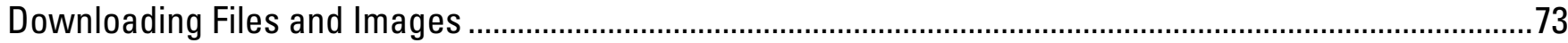

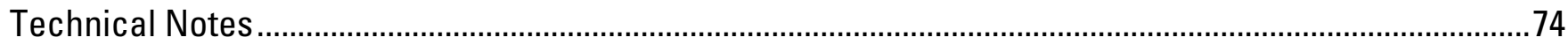




\title{
Surficial Sediment Character of the New York-New Jersey Offshore Continental Shelf Region: a GIS Compilation
}

\author{
By S. Jeffress Williams, Matthew A. Arsenault, Lawrence J. Poppe, Jane A. Reid, Jamey M. Reid,
} and Chris J. Jenkins

\section{Introduction}

\section{Overview}

Broad continental shelf regions such as the New York Bight are the product of a complex geologic history and dynamic oceanographic processes, dominated by the Holocene marine transgression ( $>100 \mathrm{~m}$ sea-level rise) following the end of the last Pleistocene ice advance $~ 20,000$ years ago. The area of the U.S. Exclusive Economic Zone (U.S. EEZ) territory, extending 200 nautical miles seaward from the coast, is larger than the continental U.S. and contains submerged landforms that provide a variety of natural functions and societal benefits, such as: critical habitats for fisheries, ship navigation and homeland security, and engineering activities (i.e. oil and gas platforms, pipeline and cable routes, potential windenergy-generation sites).

Some parts of the continental margins, particularly inner-continental shelf regions, also contain unconsolidated hard-mineral deposits such as sand and gravel that are regarded as potential aggregate resources to meet or augment needs not met by onshore deposits (Williams, 1992). The present distribution of surficial sediment off the northeastern United States is shaped from the deposits left by the last glaciation and reflects the cumulative effects of sediment erosion, transport, sorting, and deposition by storm and tidal processes during the Holocene rise in sea level. As a result, the sediments on the sea floor represent both an historical record of former conditions and a guide to possible future sedimentary environments.

The U.S. Geological Survey (USGS) through the Coastal and Marine Geology Program, in cooperation with the University of Colorado and other partners, has compiled extant sediment character and textural data as well as other geologic information on the sea floor from all regions around the U.S. into the usSEABED data system (Reid and others, 2005; Buczkowski and others, 2006; Reid and others, 2006). The usSEABED system, which contains information on sediment grain size and lithology for more than 340,500 stations within the U.S. EEZ. has been developed and populated with data as part of the USGS Marine Aggregate Resources and Processes and the National Benthic Habitats projects in order to provide the base-line data needed to update the current maps of offshore surficial geologic character and sediment distribution. The maps are also used to characterize benthic sea floor environments important for marine ecosystems. 
U.S. Geological Survey, Data Series 118 (Reid and others, 2005), of the usSEABED data release series, represents the combined efforts of the USGS and several other government agencies to provide a unified resource for accessing and preserving records of U.S. east coast sea floor geologic information and sediment texture data.

For this present report, we have chosen to focus on the New York-New Jersey region, an area that has been intensely studied by the USGS for many years to address many complex issues.

This report illustrates the uses of the usSEABED database for GIS applications, while offering additional insight into the resources and data available from the USGS and its collaborative institutions.

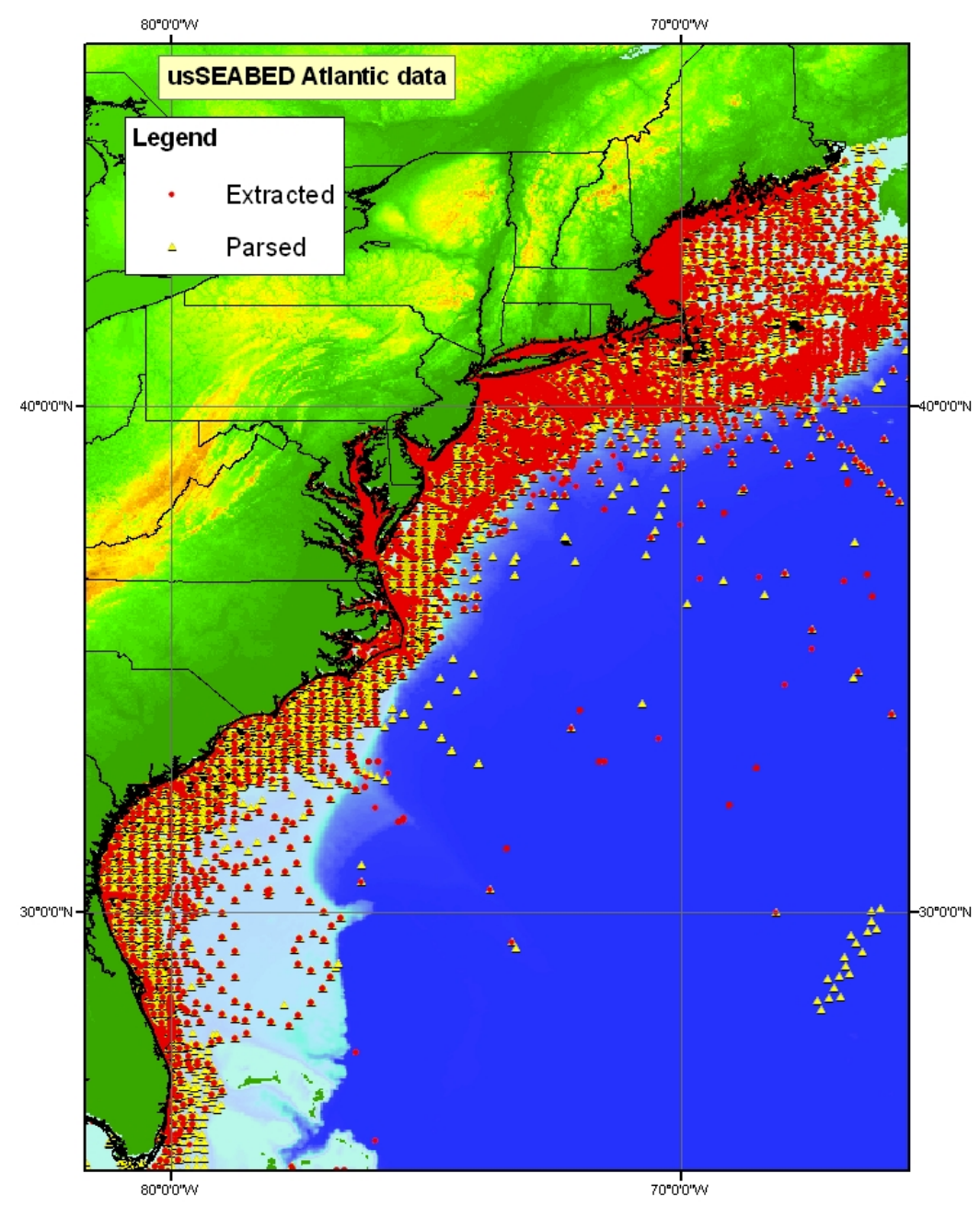

Sediment data points along the East coast of the United States (Reid and others, 2005, USSEABED; Atlantic coast offshore surficial sedient data release, U.S. Geological Survey). 
This report is based on data contained in U.S. Geological Survey Data Series 118 (Reid and others, 2005) and shows an assortment of example GIS products that are possible using usSEABED. All data are intended to be GIS-ready and should not require any additional cleanup, formatting, or renaming of fields in order to use the data in a Geographic Information System. This project employs the Environmental Systems Research Institute's (ESRI) $\mathrm{ArcView}^{\mathrm{TM}}$ software. Many of these maps were made as part of the ongoing USGS study to assess marine aggregate resources offshore New York and New Jersey, but these maps can serve many other purposes. The marine science community, educators, students and others are encouraged to use these data to generate GIS products for their own purposes.

The objectives of the Marine Aggregate Resources and Processes project are to produce a series of new geologic maps and reports of the sea floor that will provide scientific insights into the character and geologic development of U.S. continental margins and to use these maps and information to assess the potential availability of offshore sand and gravel resources. The mapping and aggregate resource assessments are being conducted on a national scale using the usSEABED data base as described in Williams and others (2003). Potential uses for these data include: (1) defining the geological variability of the sea floor in relation to benthic habitat diversity; (2) improving our understanding of the processes that control the distribution and transport of bottom sediments and benthic habitats; (3) locating aggregate resources for beach nourishment and industrial applications; and (4) providing a detailed geospatial framework for future marine science research, monitoring, and management activities. The initial assessments are in progress for the New York Bight and Louisiana offshore areas.

\section{Applications}

The usSEABED dataset has many potential uses. Discussed here are three illustrated applications of how the data may be used. Examples of maps that may be made with the data included on this CD-ROM may be found in the Browse Maps section of this publication. 


\section{Fish Habitat Mapping}

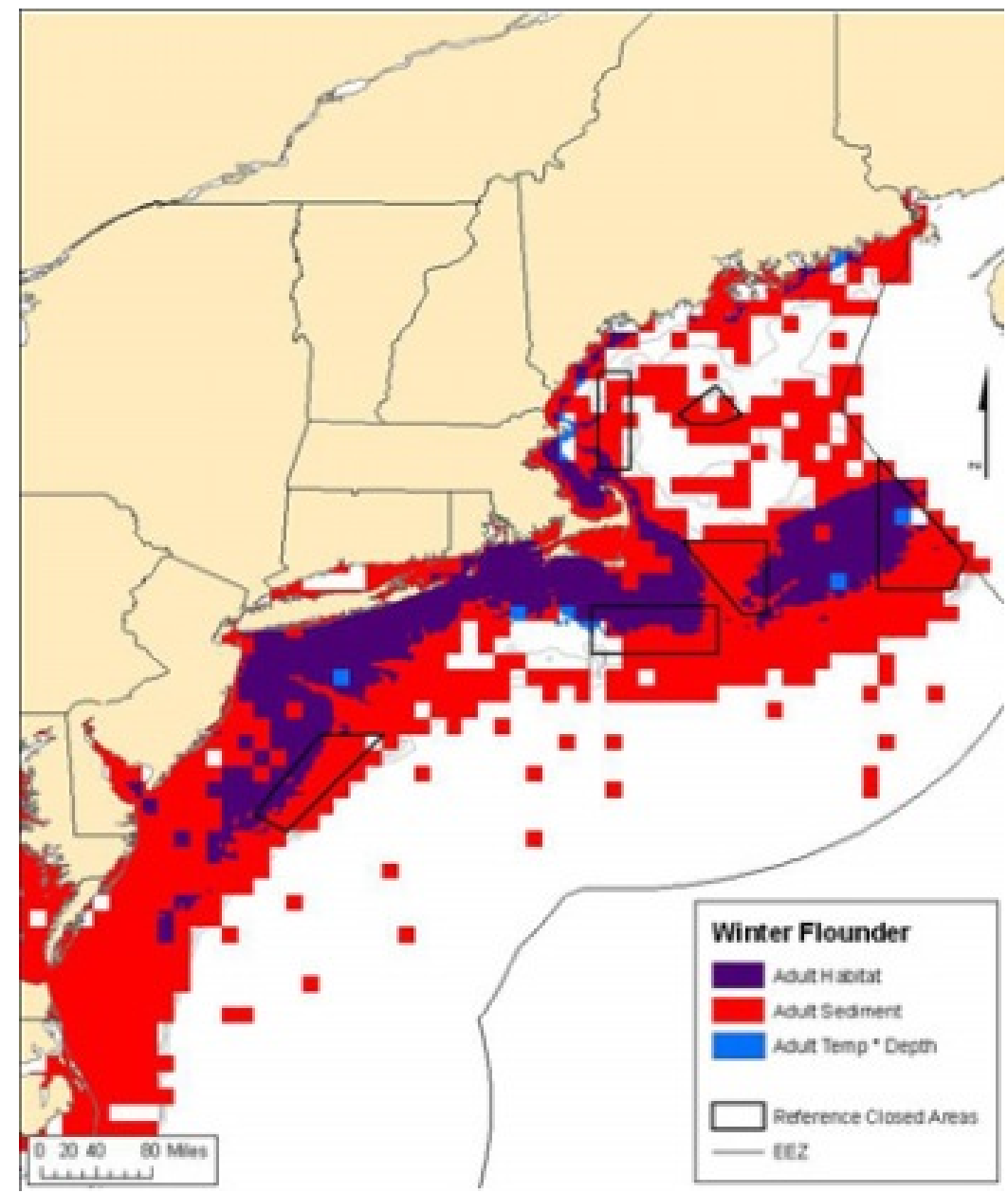

Image courtesy of Tyler Hautaniemi.

Since the passing of the Sustainable Fisheries Act of 1996, Regional Fishery Management councils around the U.S. have been describing and identifying essential fish habitat $(\mathrm{EFH})$ in their respective regions to more effectively manage the Nation's fisheries. Congress defined EFH as "those waters and substrate necessary to fish for spawning, breeding, feeding or growth to maturity."

Since late-2005, the New England Fishery Management Council's Habitat Plan Development Team, a scientific and technical body that advises the Council, has been using the usSEABED dataset correlate the relative abundance of 26 federally-managed fish species with substrate type (e.g., mud, sand, gravel, and mixtures) as a proxy for benthic habitats.

This map is a provisional attempt at depicting EFH's. Classification areas are designated over 10 minute by 10 minute squares (TMS) of latitude and longitude off the Northeast U.S. coast. The usSEABED database is used to identify the TMS that have sufficient amounts of correlated sediments in an effort to establish those areas which are potentially important for fish habitat (Tyler Hautaniemi, personal communication). 


\section{Sand and Gravel Resouces for Beach Nourishment}

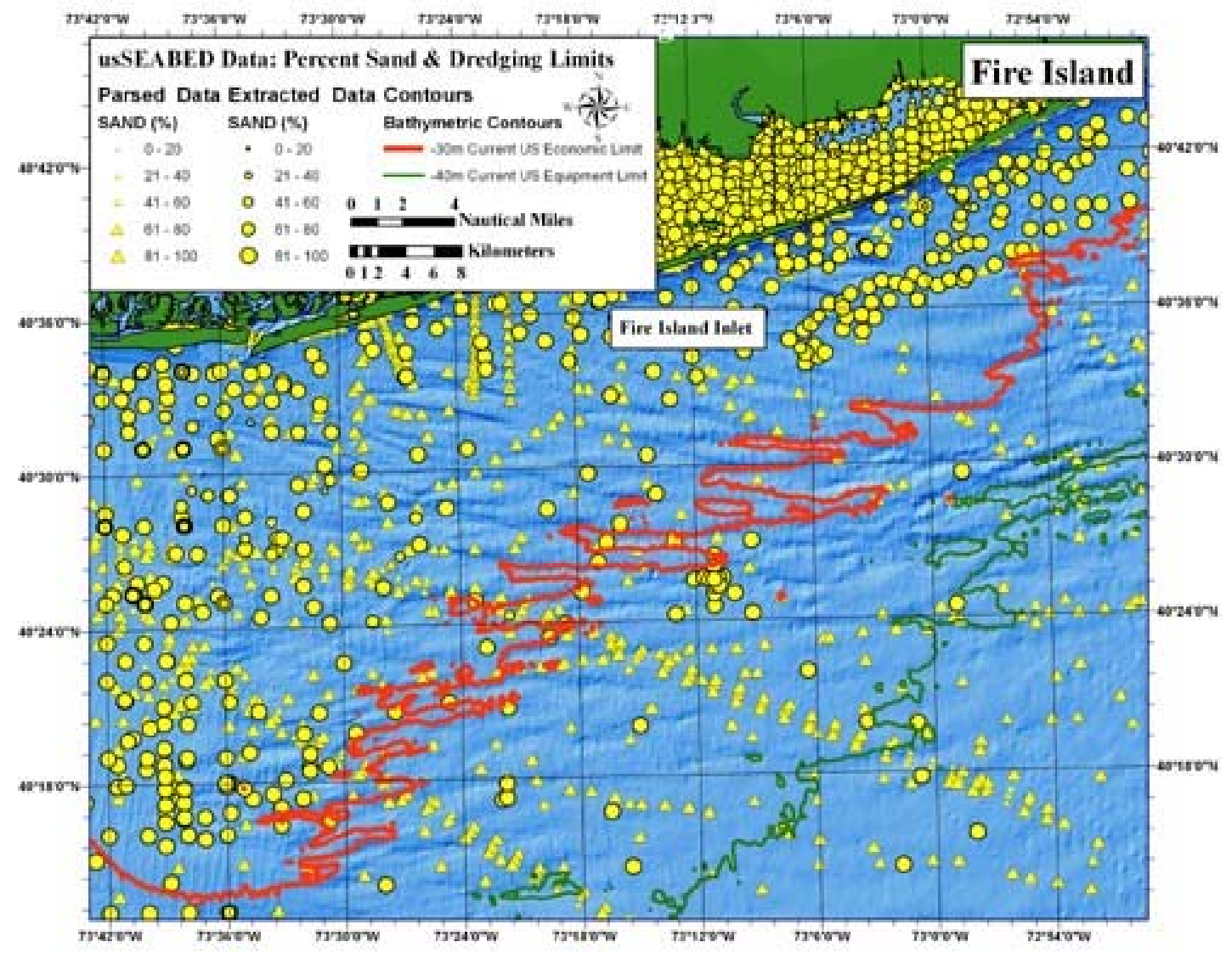

Beach nourishment, a method of dredging sand from offshore areas and pumping it ashore to widen and elevate beaches and dunes is often viewed as a cost-effective and environmentally acceptable method for use on developed coasts to mitigate erosion, reduce storm and flooding risk, enhance recreation, and restore degraded ecosystems. For beach nourishment to be viable, however, large volumes of high quality sand are necessary and the sand deposits must be located reasonably close to the beaches being nourished. Also, the sand deposits ideally should be in water depths ranging from approximately - $10 \mathrm{~m}$, an approximate "close-out depth" for nearshore sediment transport, to $-40 \mathrm{~m}$, an approximate current limit of U.S. commercial dredging. The map above shows the percent sand composition of usSEABED data points on the Long Island inner shelf in relation to the current water depth dredge limits. For beach nourishment, $>90 \%$ sand is optimal for best performance. 


\section{Planning Sea-Floor Routes and Utilities Placement}

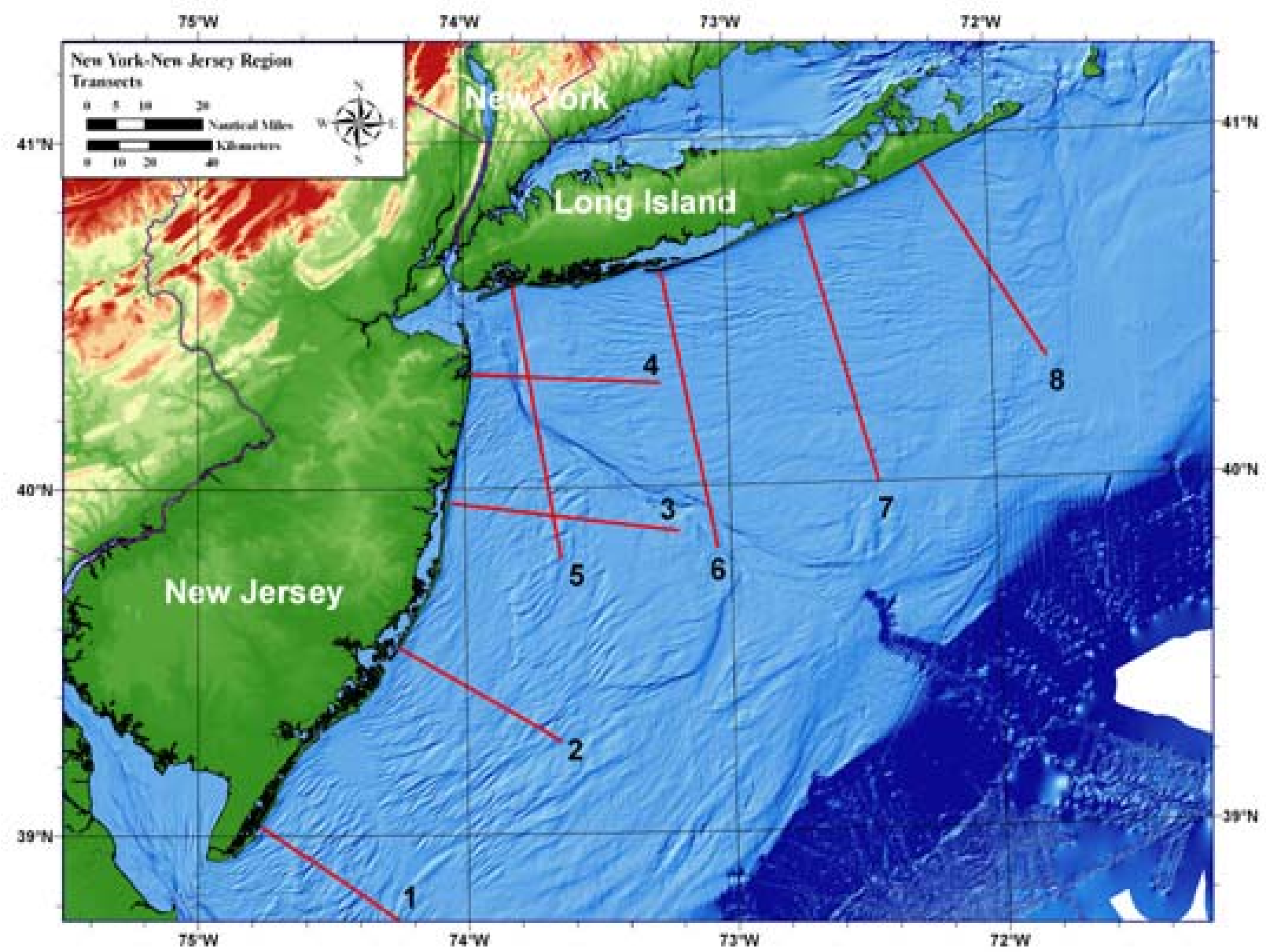

The New York Bight shelf is the product of a complex geologic history and dynamic oceanographic processes, dominated by the Holocene marine transgression during the past 20,000 years. Engineering activities (i.e., pipeline and cable routes, potential wind-energygeneration sites) are increasing within these regions as infrastructure and resource planning tries to keep pace with growing demand. 

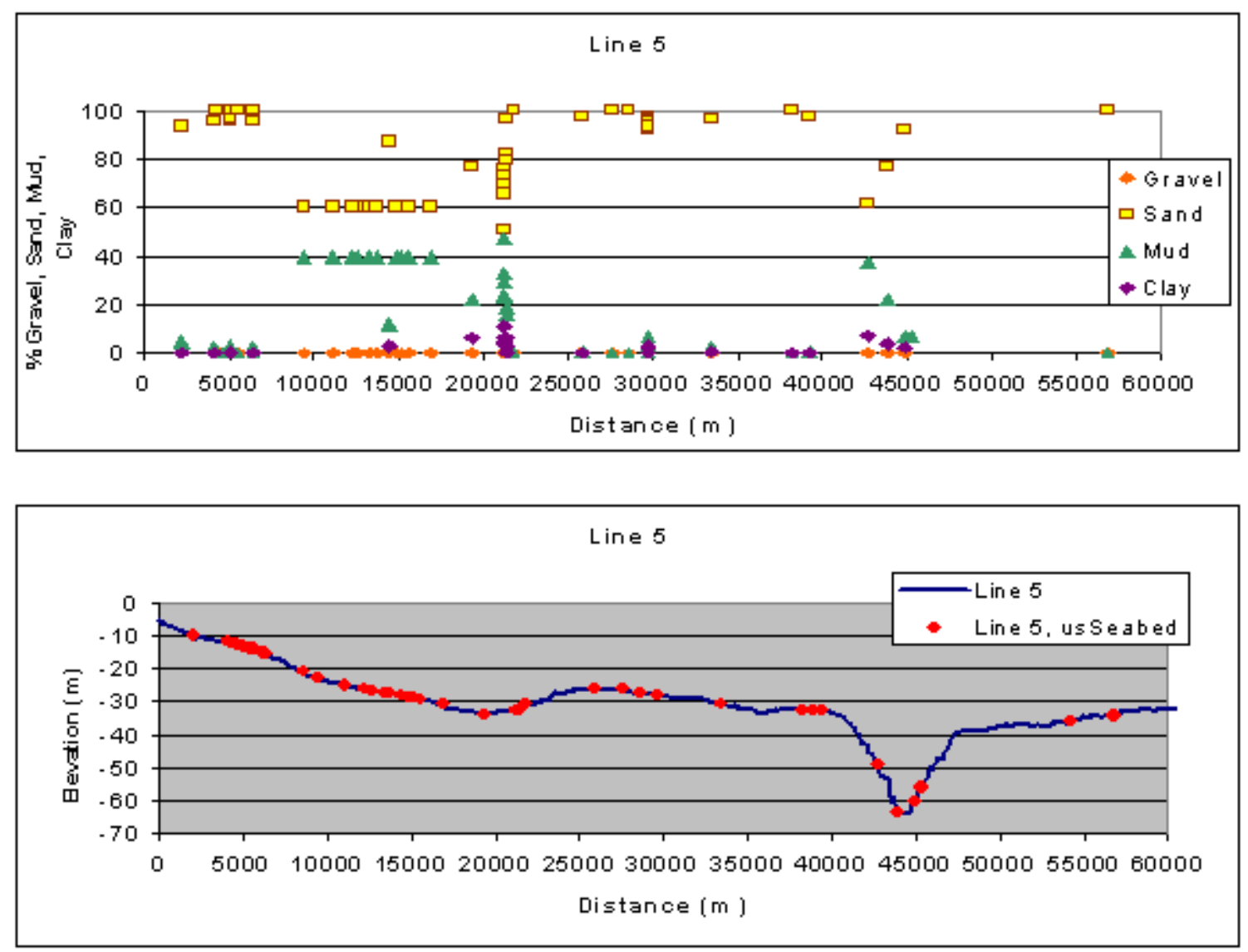

North-south transect from Rockaway Beach, NY along the NJ coast displaying sedimentary features of the geologic record, important in engineering uses of the sea floor. The sandy inner shelf extends $20 \mathrm{~km}$ offshore to $-32 \mathrm{~m}$ water depths, while the slightly elevated region $(-28 \mathrm{~m})$ farther south is in contrast to the deeply incised $(-64 \mathrm{~m})$ Hudson Shelf Valley that displays a marked asymmetry. 


\section{Nomenclature}

Nomenclature describing sediment texture distributions is important to geologists and sedimentologists because grain size is the most basic attribute of sediments. Traditionally, geologists have divided sediments into four size fractions that include gravel, sand, silt, and clay, and classified these sediments based on the dominant size fractions. Definitions of the fractions have long been standardized to the grade scale described by Wentworth (1922), and the size data compiled in this report conform to these definitions. Specifically, according to the Wentworth grade scale (PDF version) gravel-sized particles have a nominal diameter of $2 \mathrm{~mm}$; sand-sized particles have nominal diameters from $<2 \mathrm{~mm}$ to $>62.5 \mu \mathrm{m}$; silt-sized particles have nominal diameters from $<62.5 \mu \mathrm{m}$ to $>4 \mu \mathrm{m}$; and clay is $<4 \mu \mathrm{m}$.

Although several classification schemes have been adopted to describe the approximate relationship between the size fractions, most sedimentologists use one of the systems described either by Shepard (1954) or Folk $(1954,1974)$. The original scheme devised by Shepard (1954) utilized a single ternary diagram with sand, silt, and clay in the corners to graphically show the relative proportions among these three grades within a sample. This scheme, however, does not allow for sediments with significant amounts of gravel. Therefore, Shepard's classification scheme was subsequently modified by the addition of a second ternary diagram to account for the gravel fraction (Schlee, 1973). The system devised by Folk $(1954,1974)$ is also based on two triangular diagrams, but it has 21 major categories, and uses the term mud (defined as silt plus clay). The patterns within the triangles of both systems differ, as does the emphasis placed on gravel. For example, in the system described by Shepard, gravelly sediments have more than 10 percent gravel; in Folk's system, slightly gravelly sediments have as little as 0.01 percent gravel. Folk's classification scheme stresses gravel because its concentration is a function of the highest current velocity at the time of deposition, together with the maximum grain size of the detritus that is available; Shepard's classification scheme emphasizes the ratios of sand, silt, and clay because they reflect sorting and reworking (Poppe and others, 2005).

Although most source data sets in this compilation (see the Data Catalog) contain raw grain-size data, several provide only verbal descriptions of the sea-floor character. Some of these verbal descriptions are somewhat detailed, such as in the lithologic descriptions file from the USGS Continental Margin Program; others are quite abbreviated, as in the one-word descriptors supplied with the NOAA Hydrographic Database. Furthermore, most source data sets contain sediment classifications that were assigned by scientists as part of the original study. These word-based data sets have been related to numeric values for inclusion in the usSEABED data sets. Users are encouraged to review the Data Dictionary section and the usSEABED Web site for a thorough explanation.

Most of the samples compiled in this report were collected using some type of grab sampler, but some were obtained by coring of dredging. When core samples are included, or when changes in the sediment type with depth are present in a grab sample, only the analysis from the uppermost sediment type was used when mapping surficial sediment distributions. Similarly, samples collected with chain dredges are probably texturally biased and care must be taken with the use of this data.

The USGS has traditionally defined surficial samples as those sediments collected from the interval 0-2 cm below the sediment/water interface. Although many of the samples in this compilation conform to this standard, some of the studies did not define this interval or reported intervals with slightly greater bottom depths (e.g. 0-5 cm). Concerned users should consult the original source references or the metadata files provided in this report. 
Plotting routines (Matlab based) for the Shepard and Schlee classifications mentioned above, are available in the For Educators section of this publication. These routines allow users to plot their own ternary diagrams.

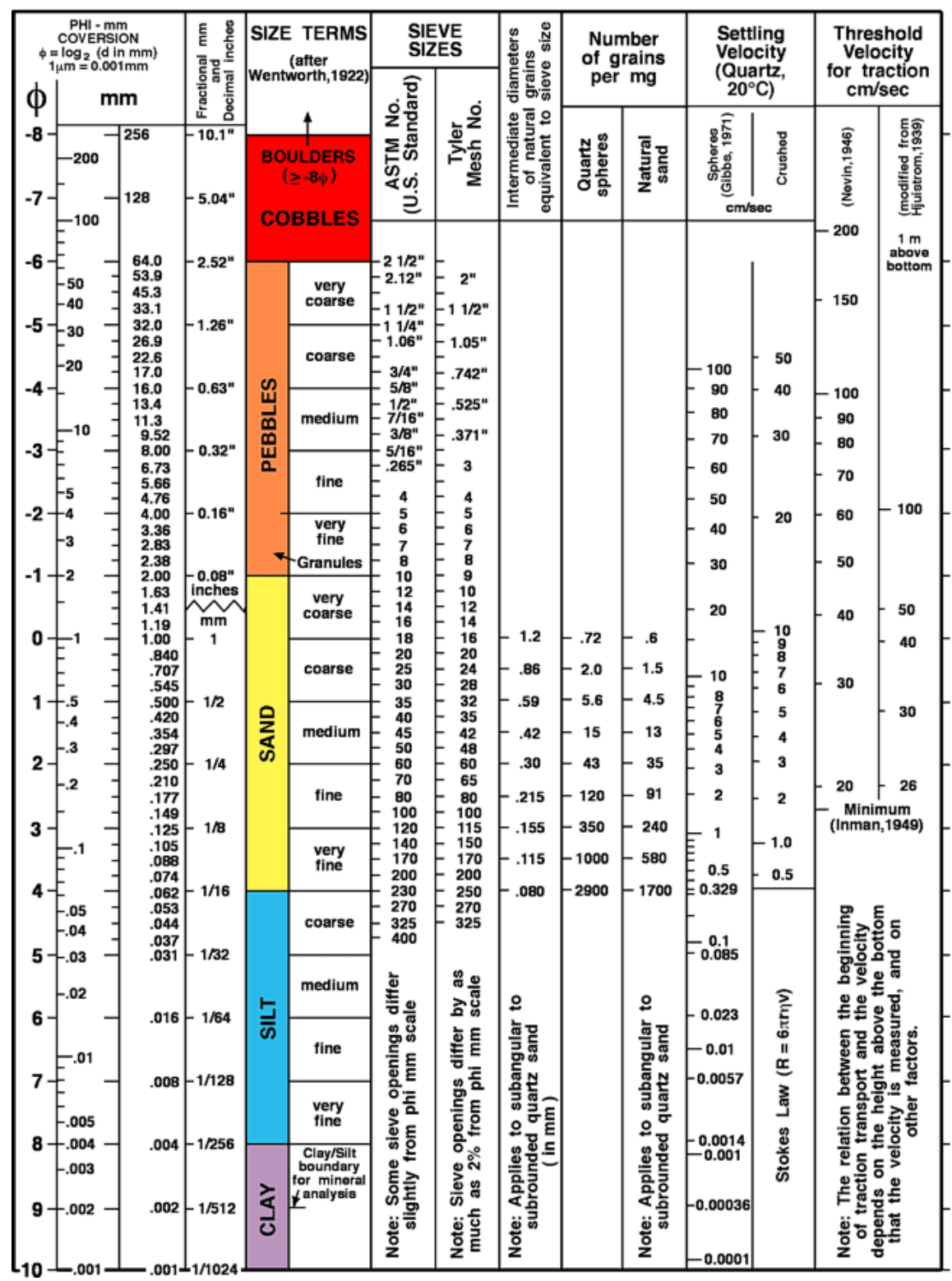

Wentworth Grade Scale 


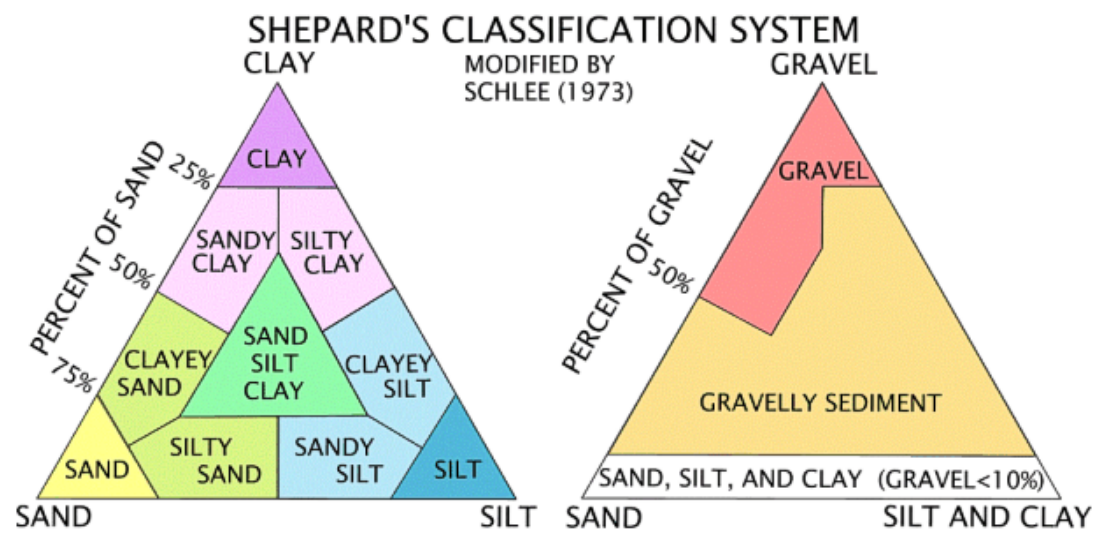

Shepard's classification system

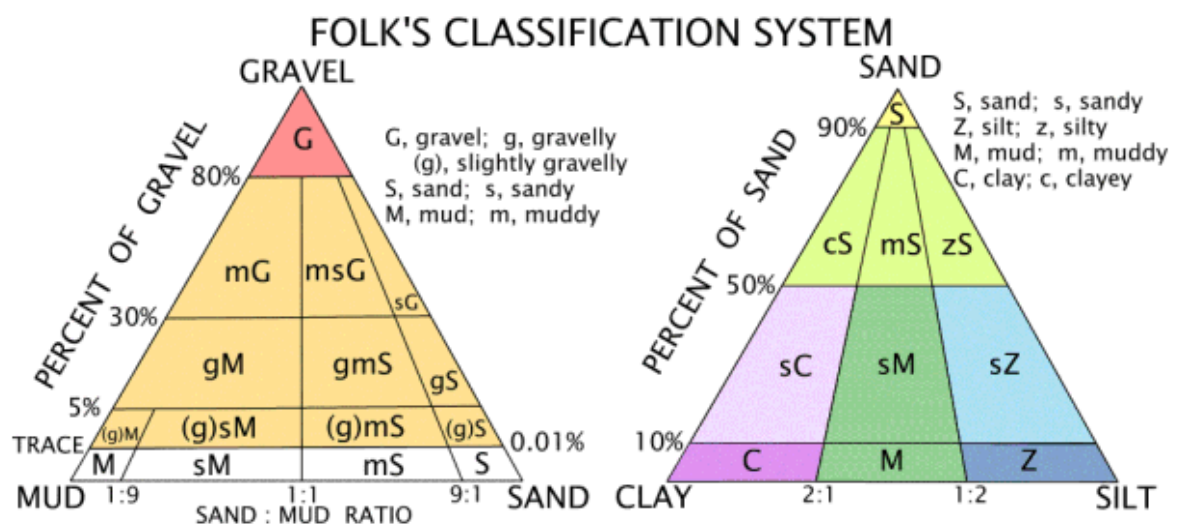

Folk's classification

\section{Sea-Floor Photos}

The New York and New Jersey region has been heavily studied by the US Geological survey, owing to its importance as one of the major recreational and industrial areas on the Atlantic seaboard. An abundance of reports on the geologic, geochemical and geophysical nature of this region are available, many of which are listed in the references section of this publication.

Much of this data is complimentary, that is to say, individual datasets and reports can be used together to present a more complete and comprehensive overview of a given area. Seafloor images are a good example of this, as good quality high-resolution photographs can aid in interpreting the geologic character of the sea floor and serve as ground truth data when used in conjunction with remotely sensed data such as sidescan sonar and multibeam hydrographic data.

The interactive image below highlights a few of the available sea-floor photos from the New York-New Jersey region, and is provided as an example of additional data that may be obtained from the U.S. Geological Survey. These photographs are from Paskevich and others (2001) OFR 2001-154, and complete photos and metadata may be downloaded from the original report (a link is provided in the data catalog). Additional imagery beyond this region is available in Butman and others (2003), OFR 2001-470. 


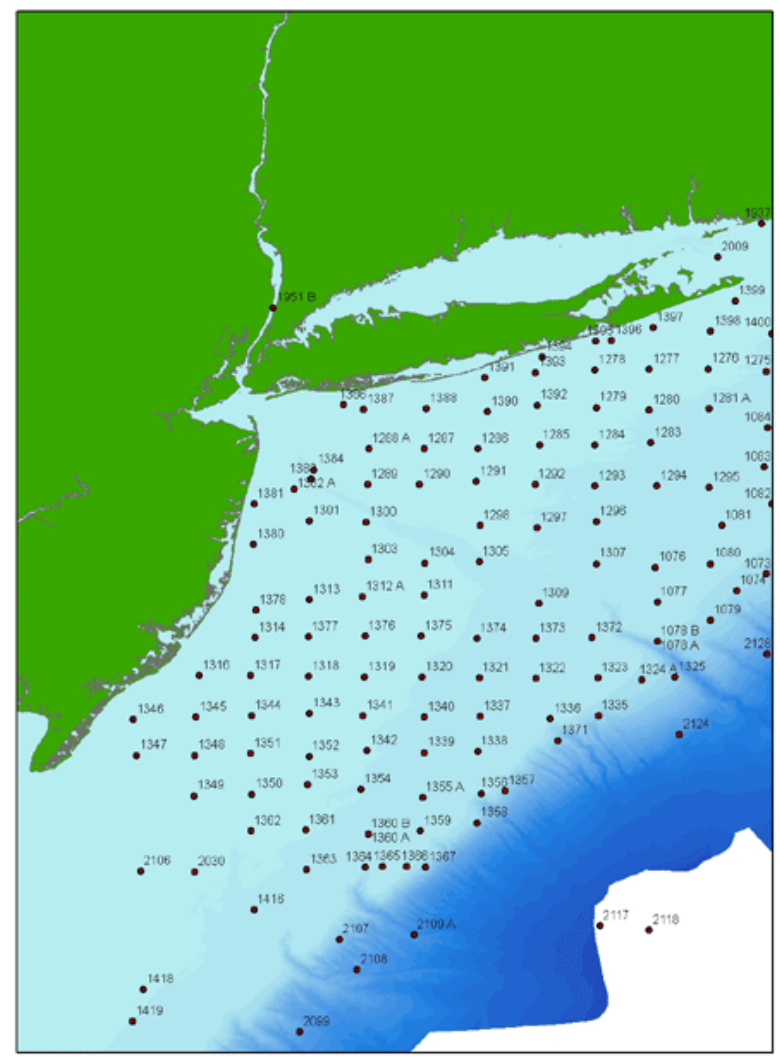

\section{Data Dictionary}

\section{Data Overview}

Geologic sedimentary character data included within this Open-File Report were previously released as USGS DS-118 (Reid and others, 2005) the first release from the usSEABED database. The database contains data and information that forms the scientific foundation for the sediment characterization found within this publication. Below you will find a description of the categories, themes and units held within the dataset.

\section{Output files}

This publication provides five usSEABED output data files for the New York-New Jersey region, and a sixth file of source data. This is only a small subset of the originally released data from USGS DS-118. 


\section{uSSEABED Output files}

$\begin{array}{ll}\text { NYB_EXT } & \text { Extracted (numeric, lab-based) } \\ \text { NYB_PRS } & \text { Parsed (word-based) } \\ \text { NYB_CLC } & \text { Calculated (calculated variables) } \\ \text { NYB_CMP } & \text { Computed (content and features) } \\ \text { NYB_FAC } & \text { Facies (components only) } \\ \text { ATL_SRC } & \text { Source File for entire Atlantic }\end{array}$

These files are downloadable from the Data Catalog.

usSEABED information is categorized into 12 data themes. A list of data themes is given in Table A. The thematic basis of the values found in the outputs can be found in field 11 ("DataType")(Table B) of the extracted (_EXT), parsed (_PRS), and calculated (_CLC) output files. Information on contribution of each source report is in the accompanying metadata files.

\begin{tabular}{|l|l|}
\hline \multicolumn{2}{|c|}{ Table A. Key to data themes in usSEABED output files } \\
\hline \hline \multicolumn{1}{|c|}{ Acronym } & \\
\hline ACU & Acoustic properties \\
\hline BIO & Biota \\
\hline CMP & Sediment composition analyses \\
\hline COL & Color \\
\hline GRZ & Grain size analysis results \\
\hline GTC & Geotechnic properties \\
\hline LTH & Lithology \\
\hline MSL & Multisensor core logger \\
\hline PET & Grain petrology \\
\hline SFT & Seafloor type descriptions \\
\hline TXG & Graphical texture statistics \\
\hline TXR & Texture statistics \\
\hline
\end{tabular}

\section{Relational keys}

The usSEABED data file types are linked relationally by the foreign keys: DataSetKey (for individual data sets), SiteKey (for individual sites), and the SampleKey (for individual analyses). The DataSetKey field gives the relationship of the data to the original source. The tables can be loaded into a relational database (RDB), relationships may be constructed, and the tables may be joined using the keys. 


\section{Source data (_SRC)}

Information about the original data are in the source (_SRC) file, including links to metadata about the original data. Each of the output data files discussed below is linked to the _SRC file by the DataSetKey field.

\section{Textural and other basic information (_EXT,_PRS,_CLC)}

Textural, statistical, geochemical, geophysical, dominant component, and color information are held in three separate, but similar, data files, based on the type of data:_EXT, _PRS, _CLC. The three data file types have the same fields (Table B) and can be combined for more extensive coverage of the sea floor. It is important for users to understand the inherent limitations of each type of file in order to choose the best data file, or combination of data files appropriate for a particular use.

\section{Extracted data (_EXT)}

The data file with the _EXT tag is the extracted data: those data from strictly performed, lab-based, numeric analyses. Most data in this file are listed as reported by the source data report; only minor unit changes are performed or assumptions made about the thickness of the sediment analyzed based on the sampler type. Typical data themes include textural classes and statistics (TXR: gravel, sand, silt, clay, mud, and various statistics), phi grain-size classes (GRZ), chemical composition (CMP), acoustic measurements (ACU), color (COL), and geotechnical parameters (GTC). The _EXT file is based on rigorous lab-determined values and forms the most reliable data sets. Limitations, however, exist due to the uncertainty of the sample tested. For example, were the analyses performed on whole samples or only on the matrix, possibly with larger particles ignored?

\section{Parsed data (_PRS)}

Numeric data obtained from verbal logs from core descriptions, shipboard notes, and (or) photographic descriptions are held in the parsed data set (_PRS). The input data are maintained using the terms employed by the original researchers and are coded using phonetically sensible terms for easier processing by dbSEABED. Longer descriptions may have the data divided by theme (Table A). The descriptions often include information on associated biota, sea floor features, and structure. Typical data themes for the parsed data set are lithologic descriptions (LTH), biology (BIO), color (COL), and (or) sea floor type (SFT, descriptions from photos or videos). The values in the parsed data file are calculated using the dbSEABED parser that assigns field values based on the form and content of a description. See the original DS-118 publication for additional information on the processing and fuzzy set theory.

The parsing process has been tested and calibrated by comparing the outputs against analytical results for the same samples. Due to the nature of visual descriptions by observers and the use of fuzzy set theory in the parser, the output data show the degree of representation in the sample, or percent abundance values. An assumption in the process is that the output degrees of representation reflect absolute abundances to some degree of accuracy. The calibrations provide information on that accuracy. Although at first sight the descriptive results in the parsed file may seem less accurate than measured values in the extracted file, they are frequently more 
representative of the sample and seabed as a whole, as they include description of objects such as shells, stones, algae, and other objects (Table C) that are a textural component of the seabed and which are often left out of laboratory analyses, particularly when a machine analysis is employed.

\section{Calculated data (_CLC)}

For the extracted and parsed data, some values are not reported by the original source, but can be calculated directly or estimated by standard derivative equations using assumptions about the conditions or variables. These values are reported in the calculated (_CLC) data files. Although the calculated ( _CLC) data can be combined with the extracted and the parsed (Table B), they are the least reliable of the three data file types and should be used with caution.

\section{Component/feature and facies data (_CMP,_FAC)}

Two usSEABED data files contain information about the presence of certain sea floor features, compositional content, biota, and sediment structure. These use major synonyms defined by the thesaurus in the dbSEABED parsing software, which clusters comparable descriptive terms together (granite represents granite, aplite, granodiorite, pegmatite, while laminated represents laminated, laminations, or lamina). Individual components and features (terms like feldspar, phosphorite, bivalves, seagrass, and wood) are held in the _CMP data file (Table D). Appropriately combined components are held in the facies (_FAC) data files (Table E). As with the parsed data files, the values held within the _CMP and _FAC files are the results of filters based on fuzzy set membership to chosen sets, and represent a measure of truth about the attribute, not percentages or defined values. These files only indicate presence, not absence, of material; it is rare that a report might state, "no bivalves" or "no phosphorite."

The _CMP file contains information about compositional content (individual minerals, rocks), genesis (terrigenous, carbonate), and certain biota. These components are internally evaluated and the value for each attribute is based solely on the relationships of attributes within the original description. The flora and fauna included in the compositional components are those that may have an effect on textural determinations in the _PRS data file, such as halimeda, bivalves, or foraminifera (Table $\mathrm{C}$ ). The values within these attribute fields range between 0 (no membership, probably due to no information), to 100 (complete membership, shell hash $=$ 100 to the shell debris set).

The _CMP file also includes information on sea floor features such as bedforms, fissures, internal structure (bedding, bioturbation), and other flora and fauna. Unlike the compositional content information, which is construed as an abundance within the sample, these attributes are an intensity of development or density of occurrence relative to scales of development or density of occurrence observed elsewhere. The flora and fauna included in the feature category are soft-bodied, for example, those that do not have an input on the textural determination within the _PRS data files, such as kelp, ophiuroids, or annelids. Values within the attribute fields range from 0 (no membership, possibly due to no information) up to $100 \%$ (maximum development). In contrast to the situation with component abundances, the sum of feature intensities in a sample is allowed to exceed $100 \%$.

The 100 most common components in the U.S. EEZ are given in the _CMP file, and those attributes with "_F" denote features. Table D lists the components and gives basic forms 
of descriptive terms that may trigger membership for each. Included in this file are 27 components that are included in the facies (_FAC) file only.

The second file, the facies file (_FAC), is created from components only, similar to the _CMP file. This file configures multiple components into appropriate groups or facies, such as igneous, metamorphic, ooze, foraminifera, and others. The dbSEABED processing software is restricted to a maximum of six components per facies. Table E lists the facies type and the components that comprise each facies group.

Again, these files only indicate presence, not absence, of material; it is rare that a report might state, "no bivalves" or "no phosphorite". The values within this attribute field range between 0 (no membership, probably due to no information), to 100 (complete membership, for example, schist $=100$ to the metamorphic set).

\section{Relationship between the _PRS and_CMP outputs}

The dbSEABED processing software recognizes that many skeletonized biota, such as halimeda, rhodoliths, shells (broken and unbroken), and others often comprise a sediment sample. Such biological terms are included in the parsing of the textural values. To see the selected biota with textural implications, see Table $\mathrm{C}$. When using the parsed data, it may be important to crosscheck with the component file using the relational foreign keys (SiteKey, SampleKey) to determine if biota are to be included in the textural outputs.

Within the_PRS file, the "seabed class" and "class membership" fields indicate the dominant compositional class and the fuzzy set membership of a sample to that class. Other components and mined information may also be listed for that sample in the _CMP file, linked by the relational keys.

\begin{tabular}{|l|l|l|l|l|}
\hline \multicolumn{1}{|c|}{ Table B. Field parameters, format, units, range, meaning, and comments for_EXT,_PRS,_CLC data } \\
files
\end{tabular}




\begin{tabular}{|c|c|c|c|c|}
\hline & & & & source metadata. \\
\hline SiteKey & $\begin{array}{l}\text { Site number } \\
\text { key }\end{array}$ & $\begin{array}{l}\text { Integer } \\
0000000\end{array}$ & For audit only & $\begin{array}{l}\text { Relational key to other data files. } \\
\text { Each site counted sequentially as } \\
\text { total output; core data may have } \\
\text { more than one sample per site. }\end{array}$ \\
\hline SampleKey & $\begin{array}{l}\text { Sample } \\
\text { number key }\end{array}$ & $\begin{array}{l}\text { Integer } \\
0000000\end{array}$ & For audit only & $\begin{array}{l}\text { Relational key to other data files. } \\
\text { Each site counted sequentially as } \\
\text { total output; Multiple samples } \\
\text { may be at each site (i.e., in core). }\end{array}$ \\
\hline Sampler & Sampler type & $\begin{array}{l}\text { Character } \\
\text { Xxxxxxxx.... }\end{array}$ & $\begin{array}{l}\text { Type of } \\
\text { sampling device }\end{array}$ & $\begin{array}{l}\text { As given in source report; } \\
\text { recovery (rcvy) or penetration } \\
\text { ('pen') length appended if given } \\
\text { in source report. For more } \\
\text { complete information on } \\
\text { sampler, see source metadata. }\end{array}$ \\
\hline DataType & Data types & $\begin{array}{l}\text { Character } \\
\text { XXX: XXX }\end{array}$ & $\begin{array}{l}\text { For audit } \\
\text { principally }\end{array}$ & Source data types (Table A). \\
\hline Gravel & Gravel & Integer 000 & $\begin{array}{l}\text { Gravel grain } \\
\text { size fraction, } \%\end{array}$ & Textural class. \\
\hline Sand & Sand & Integer 000 & $\begin{array}{l}\text { Sand grain size } \\
\text { fraction, } \%\end{array}$ & Textural class. \\
\hline Mud & Mud & Integer 000 & $\begin{array}{l}\text { Mud grain size } \\
\text { fraction, } \%\end{array}$ & Textural class. \\
\hline Clay & Clay & Integer 000 & $\begin{array}{l}\text { Clay grain size } \\
\text { fraction, } \%\end{array}$ & $\begin{array}{l}\text { Textural class; output for '_EXT' } \\
\text { only, as clay value can be } \\
\text { determined only by analysis. }\end{array}$ \\
\hline Grain size & Grain size & $\begin{array}{l}\text { Decimal } \\
00.00\end{array}$ & $\begin{array}{l}\text { Phi } \\
\text { characteristic } \\
\text { grain size }\end{array}$ & $\begin{array}{l}\text { Consensus of mean and median } \\
\text { grain sizes. }\end{array}$ \\
\hline Sorting & Sorting & Decimal 0.00 & $\begin{array}{l}\text { Phi grain size } \\
\text { dispersion }\end{array}$ & Standard deviation, sorting only. \\
\hline SeafloorClass & Seafloor class & $\begin{array}{l}\text { Character } \\
\text { Xxxxx... }\end{array}$ & $\begin{array}{l}\text { That class (or ' } \\
\text { facies ' ) with the } \\
\text { maximum fuzzy } \\
\text { membership, if } \\
\text { above } 30 \%\end{array}$ & Output for '_PRS' table only. \\
\hline ClassMbrshp & $\begin{array}{l}\text { Class } \\
\text { membership }\end{array}$ & Decimal 000 & $\begin{array}{l}\text { Fuzzy } \\
\text { membership (\%) } \\
\text { of the class (or } \\
\text { 'facies'), noted } \\
\text { above }\end{array}$ & Output for '_PRS' table only. \\
\hline $\begin{array}{l}\text { Folk } \\
\text { classification; } \\
\text { Shepard } \\
\text { classification }\end{array}$ & $\begin{array}{l}\text { Folk } \\
\text { classification; } \\
\text { Shepard } \\
\text { classification }\end{array}$ & $\begin{array}{l}\text { Character } \\
\text { xx.XX... }\end{array}$ & & \\
\hline RockMbrshp & Rock index & Integer 000 & $\begin{array}{l}\text { Fuzzy } \\
\text { membership (\%) }\end{array}$ & $\begin{array}{l}\text { Membership of sample to 'rock } \\
\text { fuzzy set'; reported only in_PRS } \\
\text { data. }\end{array}$ \\
\hline WeedMbrshp & Weed index & Integer 000 & Fuzzy & Membership of sample to 'weed \\
\hline
\end{tabular}




\begin{tabular}{|c|c|c|c|c|}
\hline & & & membership (\%) & $\begin{array}{l}\text { fuzzy set'; reported only in_PRS } \\
\text { data. }\end{array}$ \\
\hline Carbonate & Carbonate & Integer 000 & $\begin{array}{l}\% ; \text { may be } \\
\text { Fuzzy } \\
\text { membership } \\
\text { (_PRS). }\end{array}$ & \\
\hline MunsellCode & $\begin{array}{l}\text { Munsell color } \\
\text { code }\end{array}$ & $\begin{array}{l}\text { Character } \\
\text { XXXXX }\end{array}$ & $\begin{array}{l}\text { Standard } \\
\text { alphanumeric } \\
\text { coding of color } \\
\text { partitioned into } \\
\text { Hue, Value, and } \\
\text { Chroma }\end{array}$ & $\begin{array}{l}\text { Ex: '5YR 6/4', See Rock-Color } \\
\text { Chart (Geological Society of } \\
\text { America, 1991). }\end{array}$ \\
\hline OrganicCarbon & $\begin{array}{l}\text { Organic } \\
\text { carbon }\end{array}$ & Integer 000 & $\%$ & $\begin{array}{l}\text { Minimum value from } \\
\text { descriptions (PRS tables) is } \\
0.1 \% \text {. }\end{array}$ \\
\hline ShearStrength & $\begin{array}{l}\text { Log shear } \\
\text { strength }\end{array}$ & Decimal 00.0 & $\begin{array}{l}\text { kiloPascals, } \\
\text { undrained, } \\
\text { unconfined }\end{array}$ & $\begin{array}{l}\text { From a variety of } \\
\text { instrumentation. }\end{array}$ \\
\hline Porosity & Porosity & $\begin{array}{l}\text { Decimal } \\
00.00\end{array}$ & $\%$ & \\
\hline P-waveVelocity & $\begin{array}{l}\text { P-wave } \\
\text { velocity }\end{array}$ & Decimal 00.0 & $\mathrm{~m} / \mathrm{sec}$ & $\begin{array}{l}\text { Usually not corrected for } \mathrm{P} / \mathrm{T} \\
\text { effects. }\end{array}$ \\
\hline $\begin{array}{l}\text { Bottom } \\
\text { roughness }\end{array}$ & Roughness & $\begin{array}{l}\text { Decimal } \\
0000.00\end{array}$ & $\begin{array}{l}\text { Coded to } \\
\text { express the } \\
\text { height and } \\
\text { length of the } \\
\text { bottom feature } \\
\text { with greatest } \\
\text { aspect ratio }\end{array}$ & $\begin{array}{l}\text { In a coding that expresses the } \\
\text { height and length of the bottom } \\
\text { feature with greatest aspect ratio; } \\
\text { a coded output representing the } \\
\text { V:H of the roughness element } \\
\text { with greatest aspect ratio, values } \\
\text { expressed as (rounded) integer } \\
\text { log2. }\end{array}$ \\
\hline $\begin{array}{l}\text { Critical shear } \\
\text { stress }\end{array}$ & $\begin{array}{l}\text { Log critical } \\
\text { shear stress }\end{array}$ & $\begin{array}{l}\text { Decimal } \\
0000.00\end{array}$ & $\begin{array}{l}\log 10 \text { of Tau in } \\
\mathrm{kPa}\end{array}$ & $\begin{array}{l}\text { Log } 10 \text { of Tau in } \mathrm{kPa} \text {, being the } \\
\text { shear stress required to initiate } \\
\text { easily observable erosion and } \\
\text { transport, whether by traction or } \\
\text { suspension; taken from a } \\
\text { compilation of published } \\
\text { relationships ranging from large } \\
\text { boulder to muds, through a range } \\
\text { of grain shapes (eg. shell). }\end{array}$ \\
\hline Sample phase & $\begin{array}{l}\text { Where in } \\
\text { sample the } \\
\text { data are from }\end{array}$ & $\begin{array}{l}\text { Character } \\
\text { Xxxxx.... }\end{array}$ & $\begin{array}{l}\text { Where sample is } \\
\text { from }\end{array}$ & $\begin{array}{l}\text { Records whether the results are } \\
\text { for the whole, bulk sediment, or } \\
\text { just to some special part like: } \\
\text { inside a nodule, burrow-infill, } \\
\text { the sand fraction, porewater } \\
\text { (chemistry), a layer in the core, a } \\
\text { gradient observed in the core, } \\
\text { badly preserved, a layer that is } \\
\text { not properly located, or sample } \\
\text { with questionable location; also } \\
\text { may report a type of analysis or } \\
\text { observation if that is special or } \\
\text { potentially unrepresentative (for }\end{array}$ \\
\hline
\end{tabular}




\begin{tabular}{|l|l|l|l|}
\hline & & & $\begin{array}{l}\text { example, XRD, smear slide). } \\
\text { Output is as a word-based } \\
\text { description that may involve } \\
\text { numerics. This field will need to } \\
\text { be selected against when GIS } \\
\text { mappings of the bulk sedient } \\
\text { characters are being mapped. } \\
\text { Only blank entries should be } \\
\text { included in such a mapping. }\end{array}$ \\
\hline
\end{tabular}

Table C. Most frequently occurring biological components that may have textural implications (U.S. waters only)

\begin{tabular}{|l|l|l|l|l|}
\hline \hline barnacles & coralline algae & fish debris_F & pteropods & serpulids \\
\hline bivalves & corals & forams & radiolaria & shells \\
\hline brachiopods & crabs & halimeda & razor clams & sponges_F \\
\hline bryozoa & crustaceans & molluscs & reefs & worm tubes_F \\
\hline calcareous algae & diatoms & nannofossils & scaphopods & \\
\hline clypeasts & echinoids & & & \\
\hline
\end{tabular}

\section{Table D. Components (features*) processed within usSEABED}

Only the descriptive terms found in source reports are defined in the dbSEABED thesaurus. Conversely, as usSEABED uses the same thesaurus as its sister data compilations (auSEABED, goSEABED), some terms listed below may not occur within U.S. waters. Only one of possible variations are listed below, for example, laminated (laminae, lamination); mollusc (mollusk, mollusca), etc.

\begin{tabular}{|l|l|}
\hline \multicolumn{1}{|c|}{$\begin{array}{c}\text { Major } \\
\text { synonym }\end{array}$} & \multicolumn{1}{c|}{ Triggering words (word variations not included) } \\
\hline andest & andesite, augite andesite, benmoreite, trachyandesite \\
\hline anmne_F & anemone, tube anemone, cerinth, cerianthid, coryanactid \\
\hline annld_F & $\begin{array}{l}\text { annelid, arenicola, beachworm, bloodworm, bristleworm, funnelworm, nereid worm, } \\
\text { polychaete, polynoid }\end{array}$ \\
\hline aren_frm & $\begin{array}{l}\text { arenaceous foraminfera, agglutinated foramifera, ammobaculite, ammodiscus, } \\
\text { textularid foraminfera }\end{array}$ \\
\hline artif_F & $\begin{array}{l}\text { artificial, soot, anchor, brass, cinder, coal, contaminated, lumber, obstruction, } \\
\text { petroleum, oil-gas, rubber band, snag, tar, wood chip, wreck }\end{array}$ \\
\hline asterd_F & asteroid, basket star, briseaster, sea star, starfish \\
\hline barit & barite (-concretion -vein) \\
\hline baslt & basalt, diorite, metabasalt, scoria, trap rock, trachybasalt \\
\hline bioturb & bioturbation \\
\hline bitumn & bitumin \\
\hline biv & bivalve, arctica, astarte, cardium, chama, chione, chlmys, clam (-shell -flat material - \\
\hline
\end{tabular}




\begin{tabular}{|c|c|}
\hline & $\begin{array}{l}\text { hash -valves), cockle (-anadara -shell), donax, glycymeris, katalysia, lamellibranch, } \\
\text { macoma, mercenaria, mulinia, mussel (-bed -bank -shell), mya, mytilus, nucula, } \\
\text { pelecypod, quahog, rangia, seep mytilid, slipper shells, surf clam, tellina, tellinid, } \\
\text { venerid, venus clams, vesicomyid, yoldia }\end{array}$ \\
\hline bluschst & $\begin{array}{l}\text { blue schist, crossite-albite schist, crossite-quartz schist, glaucophane, quartz crossite } \\
\text { schist, quartz glaucophane schist }\end{array}$ \\
\hline bnth_frm & $\begin{array}{l}\text { benthic foraminfera, archaias, bolivina, bulimina, coralline forams, discorbis, } \\
\text { eponides, homotrema, hyaline, lenticulina, loxostema, miliolid, nodosirid, nonien, } \\
\text { notosirid, peneroplis, porcellanous, rotaiid, uvigerina }\end{array}$ \\
\hline borng_F & boring, bioeroded \\
\hline brach & brachiopod, lingula \\
\hline brncl & barnacle \\
\hline bryz & bryozoa, polyzoa \\
\hline burw_F & burrow, chondrite, clam siphon, crab hole, lebensspurren, Thalassinoides \\
\hline c_alg & calcareous algae, purple algae, red algae \\
\hline calc_ooz & $\begin{array}{l}\text { calcareous ooze, nannofossil -mud -ooze, pteropod -mud -ooze, foraminiferal -marl - } \\
\text { ooze -mud, globigerina -mud -ooze }\end{array}$ \\
\hline calcrst & calcareous crust, tufa \\
\hline calct & calcite (-cement -core -filling -veinlets) \\
\hline carb & $\begin{array}{l}\text { allogenic grain, authigenic carbonate, biogenic, calcareous, calcilutite, calcarenite, } \\
\text { calcirudite, calcareous biogenic, carbonate, limey, marl, skeletal micrite }\end{array}$ \\
\hline chrcoal_F & charcoal, fire debris \\
\hline chrt & chert, flint, porcellanite \\
\hline claymin & clay mineral, bentonite, chlorite, collophane, illite, kaolinite \\
\hline clypeast & clypeasteriod, sand dollar \\
\hline coal & coal, lignite, bituminous \\
\hline coralgl & algal coral, coralgal \\
\hline crab & crab, hermit crab, sand crab, spider crab, swimming crab \\
\hline crinod_F & crinoid, basket star \\
\hline crl & $\begin{array}{l}\text { coral, Acropora palmata, brain coral, Dendrophyllia, Madrepore, Manicina, Porite, } \\
\text { sea twig }\end{array}$ \\
\hline crl_dbr & coral debris \\
\hline crlrf & coral reef, coral heads, shingle bank, reefal shoal \\
\hline crnalg & coralline algae, calcareous algae, lithothamnion \\
\hline crustac & crustacea, decapods, lobster, shrimp shell \\
\hline defrmn_F & deformation, convolute, flame structure, flow structure, load -cast -structure, pull \\
\hline
\end{tabular}




\begin{tabular}{|c|c|}
\hline & apart \\
\hline diat & diatom, diatomite/diatomaceous \\
\hline dolmt & dolomite, ankerite, molar magnesium carbonate \\
\hline echnd & echinoid, heart urchin, keyhole urchin, sea urchin, spiny urchin \\
\hline echndrm_F & echinoderm \\
\hline fault_F & fault \\
\hline fces & feces, coprolite \\
\hline ferug & $\begin{array}{l}\text { ferruginous, iron fragment, iron (-cement -streak -flake -stain), iron stone, laterite, } \\
\text { limonite }\end{array}$ \\
\hline flasr_bed_F & flaser bed \\
\hline fld & feldspar, albite, andesine, anorthorite, K-feldspar, labradorite, orthoclase, plagioclase \\
\hline frm & calcareous foramifera, foraminifera, globigerina bit, planktonic \\
\hline gas & foamy, gas \\
\hline gbbro & $\begin{array}{l}\text { gabbro, diabase, diorite, dolerite, meta-dolerite, monzodiorite, monzonite, quartz } \\
\text { diorite }\end{array}$ \\
\hline glacl & glacial, diamicton, erratic, moraine, till \\
\hline glauc & glauconite, greensand \\
\hline gniss & gneiss, diorite gneiss, granite gneiss \\
\hline gradd_F & $\begin{array}{l}\text { coarsening upward, fining upward, increasing grain size, normally graded, reverse } \\
\text { graded }\end{array}$ \\
\hline granit & granite, aplite, granodiorite, pegmatite \\
\hline grnschst & greenschist \\
\hline gstrpd & gastropod, cerithium, conch, turitella, snail, nassarius, olivella, tenebrae, turitella \\
\hline $\mathrm{h} 2 \mathrm{~s}$ & hydrogen sulfide, hydrogen sulfide -odor -smell, sulfur odor \\
\hline halmda & halimeda, Peyssonnelia \\
\hline holoth & holothurian, sea cucumber \\
\hline hvy_min & $\begin{array}{l}\text { heavy mineral, anatase, andalusite, apatite, black sand, brookite, cassiterite, } \\
\text { clinozoisite, corundum, dumortierite, epidote, garnet, ilmenite, jadeite, kyanite, } \\
\text { leucoxene, magnetite, monazite, ore mineral, piedmontite, rutile, sillimanite, sphene, } \\
\text { spinel, staurolite, titanomagnetite, titanite, tourmaline, topaz, zircon, zoisite }\end{array}$ \\
\hline hydrt & hydrate, gas hydrate \\
\hline ign_rck & $\begin{array}{l}\text { igneous rock, acidic rock, alkali basinite, augite plagioclase porphyry, augite } \\
\text { porphyry, basic rock, dacite, felsite, olivine plagioclase clinopyroxene, olivine } \\
\text { plagioclase phyric, plagioclase andesite porphyry, plagioclase augite porphyry, } \\
\text { plagioclase porphyry, plutonic rock, porphyry, rhyolite, syenite, trachyte }\end{array}$ \\
\hline klp_F & kelp, brown algae, ecklonia, M. vertebralis, red brown algae \\
\hline
\end{tabular}




\begin{tabular}{|c|c|}
\hline lamintd_F & laminated \\
\hline lenticlr_bed & lenticular bed, lenticular mass \\
\hline limstn & $\begin{array}{l}\text { limestone, beach rock, bioclastic -floatstone - grainstone -limestone -rudstone, } \\
\text { boundstone, bryozoan - floatstone - grainstone -rudstone, calcareous - chip -rubble -rock, } \\
\text { coral limestone, floatstone, grainstone, packstone, rudstone, wackestone }\end{array}$ \\
\hline Imp_F & lump, aggregate, ball, cast, clump, compact clot, intraclast, pellet, pisolitic, peloid \\
\hline Irg_frm & $\begin{array}{l}\text { large foram, foraminferal gravel, amphistegina, heterostegina, macro foraminifera, } \\
\text { marginopora }\end{array}$ \\
\hline maf & $\begin{array}{l}\text { mafic, actinolite, aegirite, amphibole, augite, (brown- green- basaltic-) hornblende, } \\
\text { bronzite, clinopyroxene, ferromagnesian, hypersthene, olivine, orthopyroxene, } \\
\text { oxyhornblende, pyroxene, titanaugite, titaniferous, tremolite }\end{array}$ \\
\hline met & metamorphic, calcsilicate, granitized, mylonite, porphyroblast, saussurite \\
\hline methne & methane \\
\hline metlif & metalliferous \\
\hline mica & mica, biotite, chlorite, muscovite, sericite, talc \\
\hline mlsc & mollusc \\
\hline mn_crst & $\begin{array}{l}\text { manganese crust, manganese iron oxide crust, manganese nodule, manganese } \\
\text { pavement, manganese phosphate material }\end{array}$ \\
\hline mn_nod & manganese nodule, iron manganese nodule, manganese concretion, micronodule \\
\hline mnoxd & manganese oxide, iron-manganese (-coat -stain -veneer), iron-manganese oxide \\
\hline motl_F & mottle, chickwire mottle \\
\hline mudlmp_F & $\begin{array}{l}\text { mud lump, armored mud ball, silty lump, clay (-aggregate -ball -chip -clast -gall - } \\
\text { lump -mass -pod), mud (-aggregate -ball -chunk -clump -clot -clast -lump -pebble - } \\
\text { pellet, claystone -chip -gall), sandstone fragment, soft pebble, shale (-fragment - } \\
\text { concretion) }\end{array}$ \\
\hline mudstn & $\begin{array}{l}\text { mudstone, calcareous (-mudstone- siltstone), clay (-rock -shale -stone), marlstone, } \\
\text { mud (-rock -stone), pelite, shale, siliceous shale, siltstone }\end{array}$ \\
\hline nan & nannofossil coccolith, nannofossil (-coccolith -ooze), silicoflagellate \\
\hline nod_F & nodule, concretion \\
\hline odr & odor, anoxic, fetid, foul, fishy, organic, sewage, smell \\
\hline oil & oil, oil glob, oil sheen \\
\hline ool & oolite, sporbo \\
\hline $\mathrm{OOZ}$ & ooze \\
\hline ophiurd_F & ophiuroid, brittle star, ophiomusium \\
\hline orgcbn & $\begin{array}{l}\text { organic carbon, carbonaceous, organic (-streak -detritus -matter -mud -content - } \\
\text { enriched -material -part -paricle -rich -rimmed), sapropel }\end{array}$ \\
\hline oyst & oyster, jingle shell \\
\hline peat & peat, lignite \\
\hline
\end{tabular}




\begin{tabular}{|c|c|}
\hline pelag & pelagic, hemipelagic, planktic, planktonic \\
\hline phspht & phosphate, phosphorite \\
\hline pit_F & pit, crater, feeding depression, hole, pockmark, mound, resting trace \\
\hline planr_bed_F & planar bed \\
\hline plnk_frm & planktonic foramifera, globerina, globorotalid, planktic foraminfera \\
\hline plnt_F & plant, mangrove, root, vegetation, weed, root \\
\hline ptr & pteropod \\
\hline pumc & pumice, ash \\
\hline pyrt & pyrite, marcasite \\
\hline qtz & $\begin{array}{l}\text { quartz, arkosic sand, calcareous quartz sand, milky vein quartz, quartz (-content - } \\
\text { fragment -grain -granule -groundmass -mass -rich -vein -veinlet -crystal), quartzose, } \\
\text { quartzite (-cobble -gravel -pebble), sandstone (-chunk -fragment), silica }\end{array}$ \\
\hline $\mathrm{rad}$ & radiolaria \\
\hline ripl & ripple, bedform \\
\hline rlct_F & relict \\
\hline root_struct & root structure, root clast, root trace \\
\hline rzr_clm & razor clam, ensis, pinna, pteria, pelecypod \\
\hline schst & $\begin{array}{l}\text { schist, -albite -chlorite -epidote, -albite -chlorite, -albite -mica, -albite -quartz - } \\
\text { chlorite, -biotite -quartz, -chlorite -albite, -chlorite -quartz -albite, -chlorite, - } \\
\text { phyllitic, -quartz -albite, -quartz -albite -chlorite, -quartz -biotite, -quartz -chlorite }\end{array}$ \\
\hline scllp & scallop, astropecten, pecten, placopecten \\
\hline scour & scour, crag and tail, erosional, lag deposit \\
\hline scphpd & scaphopod, dentalium \\
\hline seagrs_F & $\begin{array}{l}\text { seagrass, acetabularia, amphibolis, batophora, eel grass, grass, halophila, } \\
\text { heterozostera, phyllospadix, posidonia, sargassum, strap grass, syringodium, } \\
\text { manatee grass, thallassia, zostera }\end{array}$ \\
\hline sft_alg_F & $\begin{array}{l}\text { soft algae, algal (-scum -filament -mush -strand), Bossea, green algae, macrophytic, } \\
\text { red algae }\end{array}$ \\
\hline sftcrl_F & soft coral, alcynacian, ascidian, coelentrate, gorgonian, hydrozoa \\
\hline shl & shell, shell (-bed -bank -carpet -fraction -content -material), shellfish, valves \\
\hline shl_dbr & $\begin{array}{l}\text { shell debris, shell hash, coquina, shell (-bit -conglomerate -fragments -festoon -grit - } \\
\text { lag -mash -material -piece -particle) }\end{array}$ \\
\hline shrmp_F & shrimp, amphipod, ampelisca, copepod, isopod, tanid \\
\hline sidrt & siderite, siderite nodule \\
\hline sil_ooz & siliceous ooze, siliceous mud \\
\hline
\end{tabular}




\begin{tabular}{|l|l|}
\hline slte & slate, phyllite, quartzite, metamorphic rock \\
\hline sndstn & sandstone, gritstone, graywacke, labile sandstone, sandstone reef, wacke \\
\hline sol_crl & solitary coral, cup coral, disc coral, horn coral, lophelia coral \\
\hline spng_F & $\begin{array}{l}\text { sponge, calcareous sponge, glass sponge, hard sponge, hexactinellid sponge, } \\
\text { porifera, Thalassodendron sponge }\end{array}$ \\
\hline spoil_F & spoil, brick, coke, dumped sediment \\
\hline srpul & serpulid, serpulid tube, serpulid worm tube \\
\hline sulf & sulfide, chalcopyrite \\
\hline trail_F & trail, trace (animal), track \\
\hline trrg & terrigenous, lithic, inorganic \\
\hline umafic & $\begin{array}{l}\text { ultramafic, amphibolite, anorthosite, dunite, greenstone, harzburgite, lherzolite, } \\
\text { norite, orthopyroxenite, periodotite, picrite, pyroxenite, serpentinite, troctolite, } \\
\text { wehrlite }\end{array}$ \\
\hline vol_rck & volcanic rock, volcanic (-cobble -pebble) \\
\hline volgls & volcanic glass, obsidian, hyaloclastite, pyroclastic, quenched, vitric, subvitreous \\
\hline volrck & volcanic rock, welded tuff \\
\hline volsed & volcanic sediment, ash, tuff, lapilli \\
\hline wood & wood, bark, twig \\
\hline wrm_F & $\begin{array}{l}\text { worm, chordate, echiurid, fan -flat (-glob -juicy -long -sand -tube worm), maldanid, } \\
\text { pogonophora, priapulida, sliverfish, siphunculid, tunicate }\end{array}$ \\
\hline wrm_tbe_F & $\begin{array}{l}\text { worm tube, agglutinated worm tube, amphipod tube, annelid worm tube, arenicola, } \\
\text { chitinous worm tube, diopatra worm tube, polychaete worm tube, pogonophoran } \\
\text { tube worm, vestimentiform tube worm }\end{array}$ \\
\hline wavy_bed_F & wavy bed \\
\hline
\end{tabular}

\section{Table E. Facies and their component makeup}

Facies values are determined by a combination of components and their mined values from wordbased descriptions. Numeric textural, geochemical, and geophysical information held in _PRS data files. Values represent memberships to fuzzy sets, given as percents. A minimum of $30 \%$ component presence is required to trip a given facies, and a component may trip more than one facies. See _FAC files for actual data, and table $\mathrm{C}$ for component information. Facies notes presence only, not absence.

\begin{tabular}{|l|l|l|l|}
\hline \hline \multicolumn{1}{|c|}{ Field name } & Parameter & $\begin{array}{c}\text { Data format, } \\
\text { units }\end{array}$ & \multicolumn{1}{|c|}{ Information/triggering components } \\
\hline Latitude* & Latitude & $\begin{array}{l}\text { Decimal } \\
00.00000\end{array}$ & Decimal degrees, $90^{\circ}$ to $-90^{\circ}$ range \\
\hline Longitude* & Longitude & $\begin{array}{l}\text { Decimal } \\
000.00000\end{array}$ & Decimal degrees, $-180^{\circ}$ to $180^{\circ}$ range \\
\hline
\end{tabular}




\begin{tabular}{|c|c|c|c|}
\hline WaterDepth* & Water depth & $\begin{array}{l}\text { Integer } \\
00000\end{array}$ & Meters \\
\hline SampleTop* & Sample top & $\begin{array}{l}\text { Decimal } \\
000.00\end{array}$ & Meters below seabed surface \\
\hline SampleBase* & Sample base & $\begin{array}{l}\text { Decimal } \\
000.00\end{array}$ & Meters below seabed surface \\
\hline SiteName* & Site name & $\begin{array}{l}\text { Character } \\
\text { XXX: XXX }\end{array}$ & Survey or laboratory code for the sampling site \\
\hline DataSetKey* & $\begin{array}{l}\text { Dataset number } \\
\text { key }\end{array}$ & Integer 000 & $\begin{array}{l}\text { Relational key to_SRC file; _SRC file contains } \\
\text { links to source metadata }\end{array}$ \\
\hline SiteKey* & Site number key & $\begin{array}{l}\text { Integer } \\
0000000\end{array}$ & $\begin{array}{l}\text { Relational key to other data files. Each site } \\
\text { counted sequentially as total output; core data } \\
\text { may have more than one sample per site. }\end{array}$ \\
\hline SampleKey* & $\begin{array}{l}\text { Sample number } \\
\text { key }\end{array}$ & $\begin{array}{l}\text { Integer } \\
0000000\end{array}$ & $\begin{array}{l}\text { Relational key to other data files. Each site } \\
\text { counted sequentially as total output; Multiple } \\
\text { samples may be at each site (i.e., in core). }\end{array}$ \\
\hline Terrigenous & Terrigenous & $(\%)$ & Fld, hvy_min, maf, mica, qtz, trrg \\
\hline Carbonate & Carbonate & $(\%)$ & Calcrst, calct, carb, dolmt, limstn, sidrt \\
\hline Igneous & Igneous & $(\%)$ & Andest, baslt, gbbro, granit, ign_rck, umafic \\
\hline Volcanic & Volcanic & $(\%)$ & Baslt, pumc, vol_rck, volgls, volrck, volsed \\
\hline Metamorphic & Metamorphic & $(\%)$ & Bluschst, gniss, grnschst, met, schst, slte \\
\hline Mineralized & Mineralized & $(\%)$ & Barit, metlif, phspht, pyrt, sulf \\
\hline AuthFeMn & $\begin{array}{l}\text { Authigenic Fe } \\
\mathrm{Mn}\end{array}$ & $(\%)$ & Mn_crust, mn_nod, mnoxd, ferug \\
\hline Ooze & Ooze & $(\%)$ & Ooz, calc_ooz, sil_ooz \\
\hline Carbon & Carbon & $(\%)$ & Coal, bitumn, orgcbn, peat \\
\hline GeochemSignal & $\begin{array}{l}\text { Geochemical } \\
\text { signal }\end{array}$ & $(\%)$ & Gas, h2s, hydrt, methne, odr, oil \\
\hline Forams & Forams & $(\%)$ & Aren_frm, bnth_frm, frm, lrg_frm, plnk_frm \\
\hline OtherCalcPelag & $\begin{array}{l}\text { Other } \\
\text { calcareous } \\
\text { pelagics }\end{array}$ & $(\%)$ & Nan, ptr, calc_ooz \\
\hline SilcPelag & $\begin{array}{l}\text { Siliceous } \\
\text { pelagics }\end{array}$ & $(\%)$ & Diat, rad, sil_ooz \\
\hline Shell & Shell & $(\%)$ & Shl, shl_dbr \\
\hline Coral & Coral & $(\%)$ & Crl, crl_dbr, crlrf, sol_crl \\
\hline HardPlant & Hard plants & $(\%)$ & C_alg, coralgl, crnalg, halmda \\
\hline
\end{tabular}




\section{Data Catalog}

Data provided in this publication are located using geographic coordinates for integration into a Geographic Information System (GIS). A GIS is defined as a system of hardware and software to support the display, manipulation, and analysis of spatial data for mapping and complex data solving. This integrated package provides researchers the ability to integrate, analyze, and map the various data sets as an aid to scientific research, and policy-making decisions tied to the environment.

The sediment/sea-floor layers contained in five shapefiles as parsed, exracted, calculated, facies and computed data. The five individual sediment/sea-floor data layers basemap data have been compiled into an ArcView TM project file (nynj.apr) at the top-level directory of this publication. This project file is intended to be opened and the data viewed with the Environmental Systems Research Institute, Inc. (ESRI) ArcView ${ }^{\mathrm{TM}}$ or ArcGIS ${ }^{\mathrm{TM}}$ software. The project file reflects relative paths to the archived data as organized within this publication. To open the supplied project file and view the datasets, the user must start the GIS software, navigate to the top-level of this publication and open the project file (nynj.apr).

For those who don't have the ESRI software or a compatible GIS data browser available on their computer, a free viewer, ArcExplorer ${ }^{\mathrm{TM}}$, is available from ESRI. Please note that the ArcExplorer ${ }^{\mathrm{TM}}$ software is limited to the Microsoft Windows operating system.

Each GIS data layer from this publication is cataloged in the table below for easy access. The individual data layers are described and include the shapefile name (e.g. EXT is extracted data ) which is linked to a browse graphic showing the data layer extent and coverage.

Federal Geographic Data Committee (FGDC) metadata for the individual data layers is provided in three versions (HTML, FAQ, and text). Selecting associated metadata files from the table below will open the information in a new browser window.

A 'zip' compressed, downloadable archive file containing the ArcView ${ }^{\mathrm{TM}}$ shapefile for each data layer is also provided. Compressed downloadable files were created using the Windows program WINZIP v8.0. For those users who do not have software capable of uncompressing the archived zip files, they may obtain a free version of the software from Winzip Computing, Inc. or Pkware, Inc. In addition to the ArcView ${ }^{\mathrm{TM}}$ shapefile, the sediment data layers are available in an ASCII text format and a Microsoft Excel spreadsheet format. The first record of the ASCII file and Excel spreadsheet contains the name of the data fields for that file.

\section{usSEABED Data}

Directory: data/usseabed

\begin{tabular}{|c|c|c|}
\hline $\begin{array}{l}\text { Data Layer Name } \\
\text { and Description }\end{array}$ & Metadata & Files \\
\hline $\begin{array}{l}\text { NYNJ_PRS - usSEABED parsed (word-based) data for the New } \\
\text { York-New Jersey region. An explanation of the individual data } \\
\text { sets may be found in the Data Dictionary section. }\end{array}$ & $\begin{array}{l}\text { HTML } \\
\text { FAQ } \\
\text { text }\end{array}$ & $\begin{array}{l}\text { zip } \\
\text { Excel } \\
\text { text }\end{array}$ \\
\hline $\begin{array}{l}\text { NYNJ_EXT - usSEABED extracted data for the New York-New } \\
\text { Jersey region. An explanation of the individual data sets may be } \\
\text { found in the Data Dictionary section. }\end{array}$ & $\begin{array}{l}\text { HTML } \\
\text { FAQ } \\
\text { text }\end{array}$ & $\begin{array}{l}\text { zip } \\
\text { Excel } \\
\text { text }\end{array}$ \\
\hline
\end{tabular}


NYNJ_CLC - usSEABED calculated data for the New YorkNew Jersey region. An explanation of the individual data sets may be found in the Data Dictionary section.

NYNJ_FAC - usSEABED facies data for the New York-New Jersey region. An explanation of the individual data sets may be found in the Data Dictionary section.

NYNJ_CMP - usSEABED computed data for the New YorkNew Jersey region. An explanation of the individual data sets may be found in the Data Dictionary section.
HTML zip

FAQ Excel

text text

HTML zip

FAQ Excel

text text

HTML zip

FAQ Excel

text text

ATL_SRC - usSEABED source data for the Atlantic Coast. An explanation of the individual data sets may be found in the Data Dictionary section.

\section{Bathymetry}

Directory: data/bathymetry

Data Layer Name
and Description

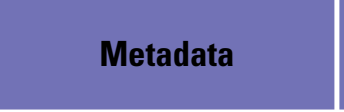

HTML

FAQ

text

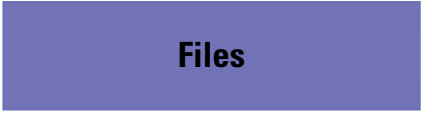

zip

converted to 'hillshaded' to show bathymetry features.

\section{Basemaps}

Directory: data/basemaps

\begin{tabular}{c}
$\begin{array}{c}\text { Data Layer Name } \\
\text { and Description }\end{array}$ \\
\hline
\end{tabular}

eez - Boundaries of the U.S. Exclusive Economic Zone (US EEZ) of the New York-New Jersey project area, extending 200 nautical miles offshore.

3nm Federal - The 3 nautical mile boundary between state and federal waters .

state_bounds - Internal US state boundaries

nos80k - US states map

\begin{tabular}{|c|c|}
\hline Metadata & Files \\
\hline $\begin{array}{l}\text { HTML } \\
\text { FAQ } \\
\text { text }\end{array}$ & zip \\
\hline
\end{tabular}

HTML

FAQ zip

text

HTML

FAQ

text

zip

HTML

FAQ zip

text 


\section{Sea-Floor Photos}

Directory: Online

\begin{tabular}{l|l|l}
\multicolumn{1}{|c|}{$\begin{array}{l}\text { Data Layer Name } \\
\text { and Description }\end{array}$} & \multicolumn{1}{|c|}{ Metadata } & Files \\
\hline $\begin{array}{l}\text { seafloor - Sea-Floor photos, previously included in USGS } \\
\text { Open-File Report 2001-154 (Paskevich and others, } \\
\mathbf{2 0 0 1}\end{array}$ & Online & Online \\
\hline
\end{tabular}

\section{Spatial and Temporal Uncertainties}

Users of usSEABED data are reminded that many sea-floor regions are, by their nature, dynamic environments subject to a variety of physical processes, such as erosion, winnowing, reworking, and sedimentation or accretion that vary on different spatial and temporal scales, and sea-floor samples may represent a only moment in time. Although usSEABED is comprised of samples collected, described, and analyzed by many different organizations and individuals over a span of years, metadata are provided for each source report. In cases where original metadata are not available from the data source, metadata were created based on available information accompanying the data. Of particular importance, site locations are as given in the original sources, with uncertainties due to navigational techniques and datums ignored in the usSEABED compilation. As many reports are decades old, users of usSEABED should use their own criteria to determine the appropriateness of data from each source report for their particular purpose and scale of interest.

In addition, there are uncertainties in data quality associated with both the extracted data (analytical analyses) and parsed data (word-based descriptions). It may be that grain-size analyses are done solely on the sand fraction, excluding coarser material, such as shell fragments and gravel, while word descriptions of sediment samples may emphasize or deemphasize the proportion of fine or coarse sediment fraction or disregard other important textural or biological components. Detailed information about issues such as these are noted in the source metadata files, and known incomplete data are decommissioned in usSEABED.

Users are encouraged to view the entire document before downloading the data files and should refer to the metadata files for information about individual sources, limitations, date of collection, and other information. As issues about the data or the data processing may be discovered, errata will be posted on the usSEABED Web site. Corrections will be included in the next version of the publication.

\section{Browse Maps}

\section{Overview}

Several examples of the maps and data products are shown below. For this publication we have chosen to focus on the Extracted and Parsed data within the dataset. The data are in no way limited to these few displays, and users are encouraged to review the definitions of the data files in the Data Dictionary (data_dictionary.htm).

The images and maps in this Open-File Report serve as examples of products that may be generated using the usSEABED data provided in the Data Catalog section. A wide array of additional gridded maps and plots of sea floor sediment texture, character, and related 
geologic attributes from the New York-New Jersey offshore region can be constructed from the usSEABED data using Geographic Information Systems (GIS) applications (e.g. ArcView ${ }^{\mathrm{TM}}$, ArcExplorer $^{\mathrm{TM}}$, MapInfo ${ }^{\mathrm{TM}}$ ).

\section{Data at a Glance}

Scatter plots of mean grain size (x-axis) vs. sorting (y-axis) for the Extracted data (top figure) and the Parsed data along the Atlantic coastal margin of the United States from USGS Data Series 118, Reid and others, 2005. Based on the sediment texture and character the samples are grouped into three primary facies: (A) Relict Cretaceous/ Pleistocene sediments, (B) Modern sand and gravel derived and reworked from older formations, and (C) Muds and muddy fine sands associated with estuarine outcrops on the shelf and the Hudson Shelf Valley. 


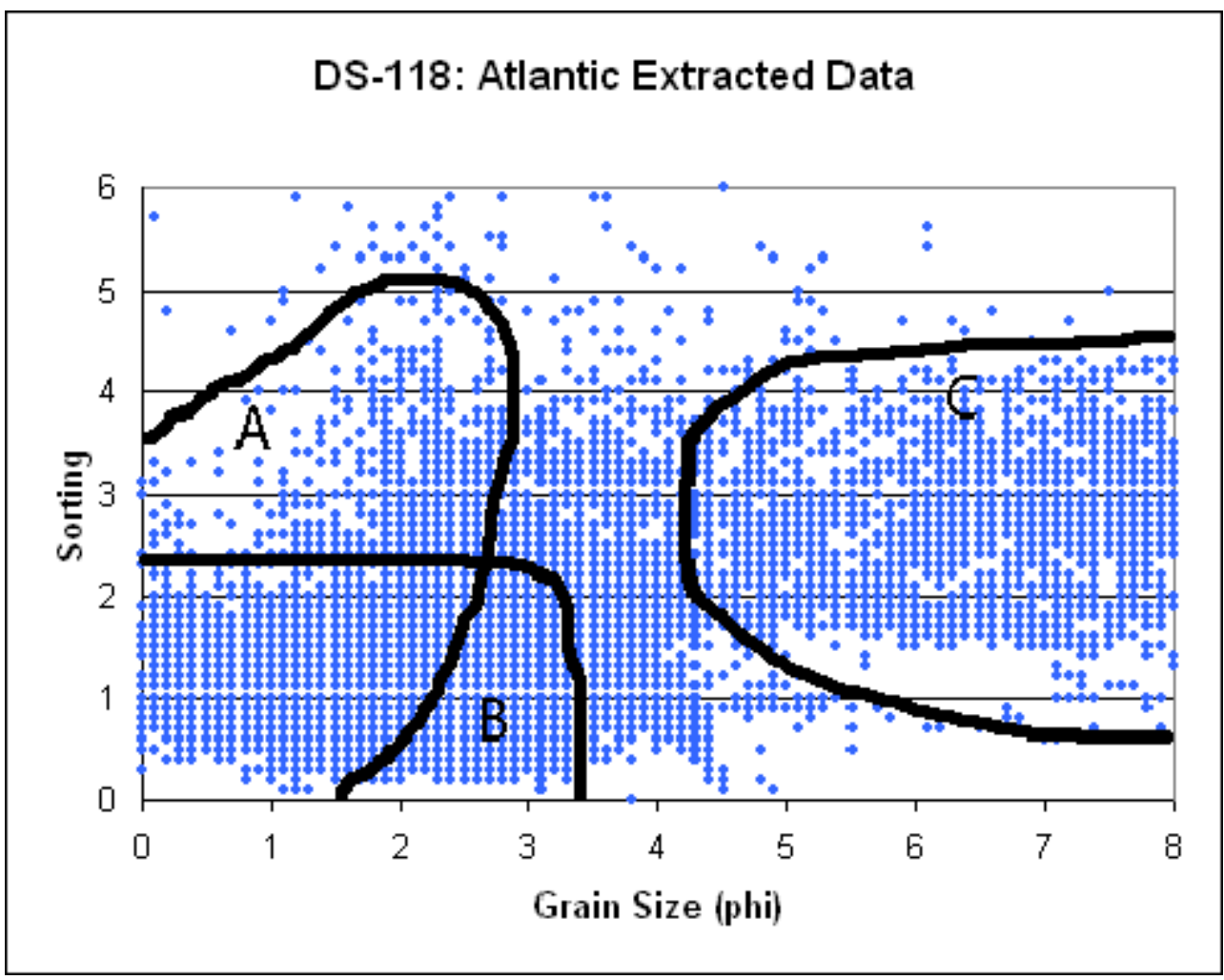

Scatter plots of mean grain size (x-axis) vs. sorting (y-axis) for the Extracted data.

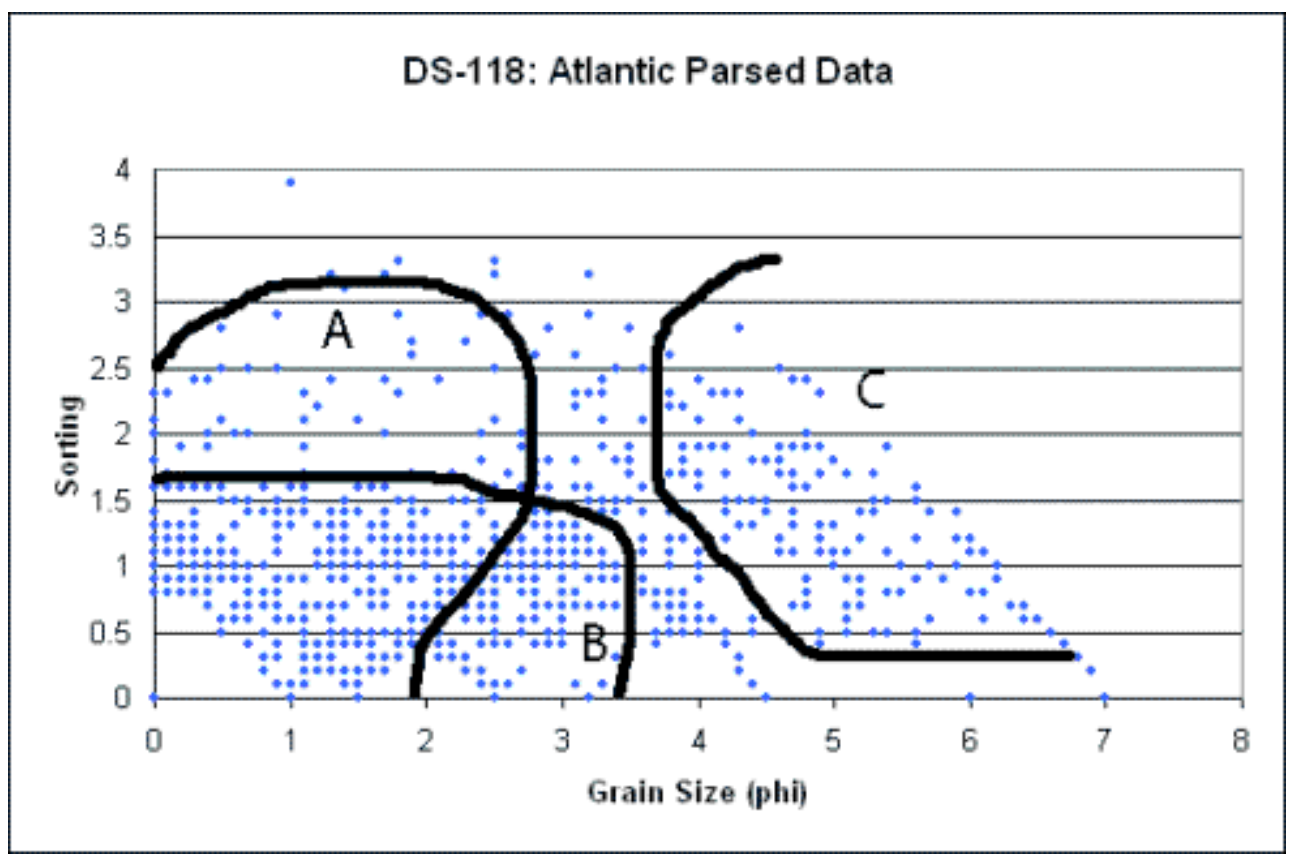

Parsed data along the Atlantic coastal margin of the United States from USGS Data Series 118, Reid and others, 2005. 


\section{Mean Grain Size}

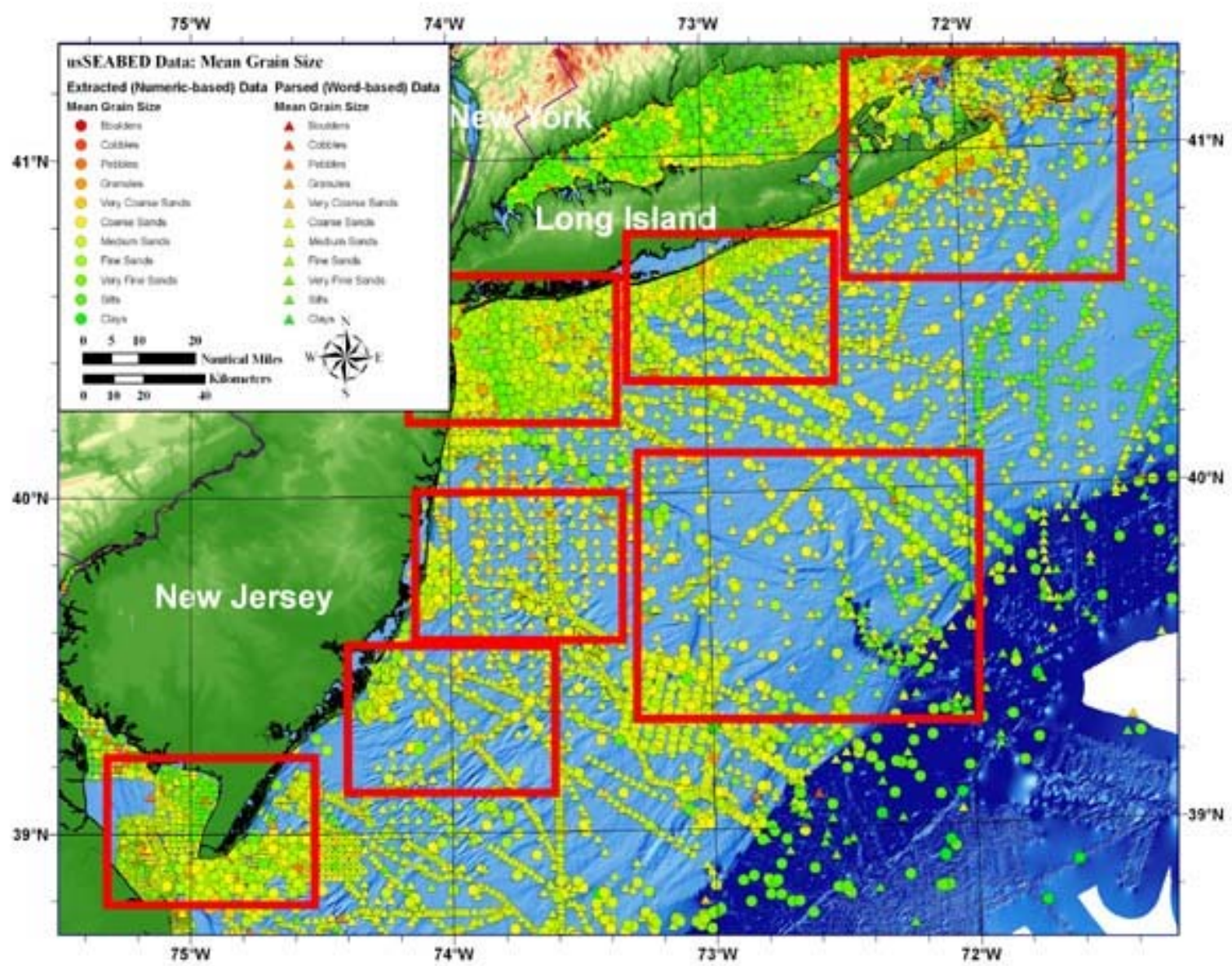

The map above of mean grain size is based on the extracted and parsed data from the usSEABED dataset (Reid and others, 2005). A larger version of the map may be viewed by selecting this link: main map. A precursory look at the map shows overall trends in sediment distribution. Long Island inner shelf regions (detail map) trend towards fine to medium sands (1-3 phi), while regions off northern New Jersey at the head of the Hudson Shelf Valley and down the thalweg tend toward finer grained sediments (>3phi) (detailed map). A predominance of fine grained sediments (muddy fine sand, mud) is also true of regions of Delaware Bay (detailed map) and outer edges of continental shelf (detailed map). Detailed maps of the areas below may be viewed by selecting the area within the red boxes.

Images are best viewed at full size. The images included in this publication are designed to be printed on $8.5^{\prime \prime} \times 11 "$ sheets of paper, and therefor are best viewed on screen at full resolution. 


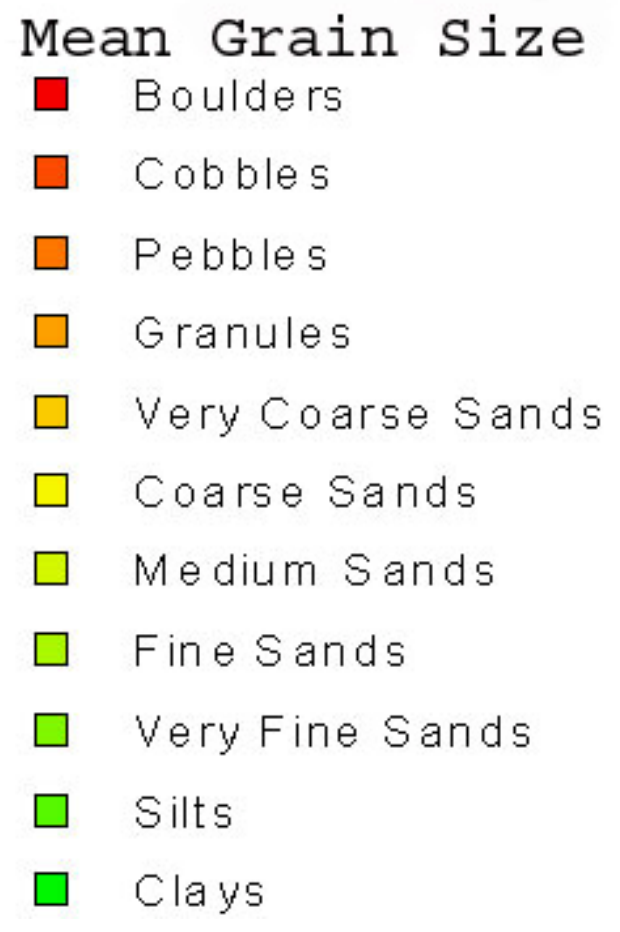

Within the usSEABED dataset, mean grain size represents the calculated average phi size from standard lab-based sample methods (extracted data) and word-based (parsed data) observational data.

Color coding is used to illustrate the surficial sediment texture and distribution documented by the point coverages. Samples for which mean grain size data are available were assigned a modified Wentworth classification, shown to the left. This is based on phi value divisions Wentworth, 1922.

\section{A Few Example Maps of Sediment Texture}

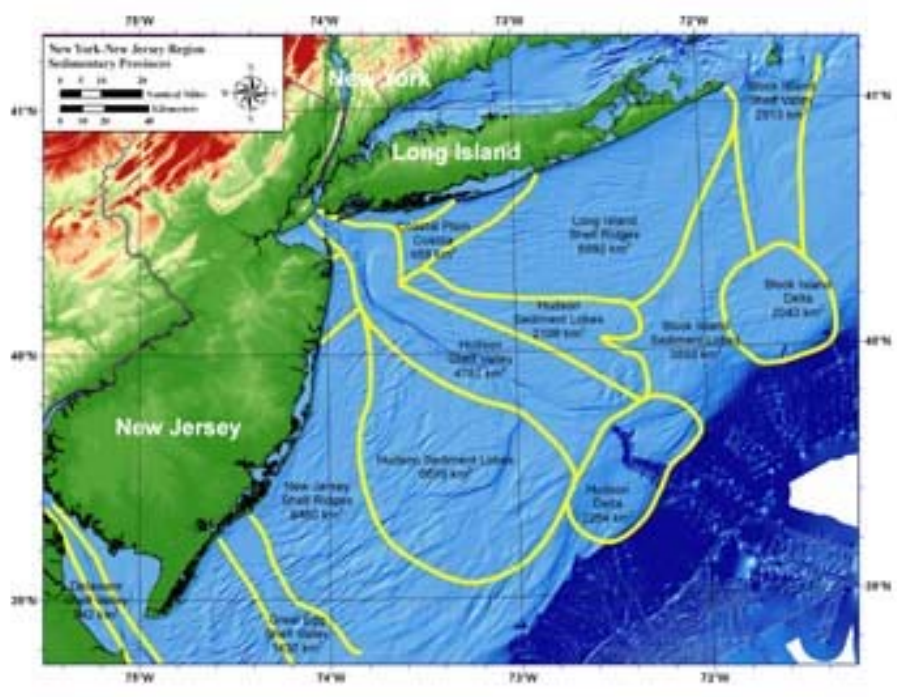

Map of the primary sedimentary provinces based on the underlying framework geology and sedimentary features comprising the sea floor. 


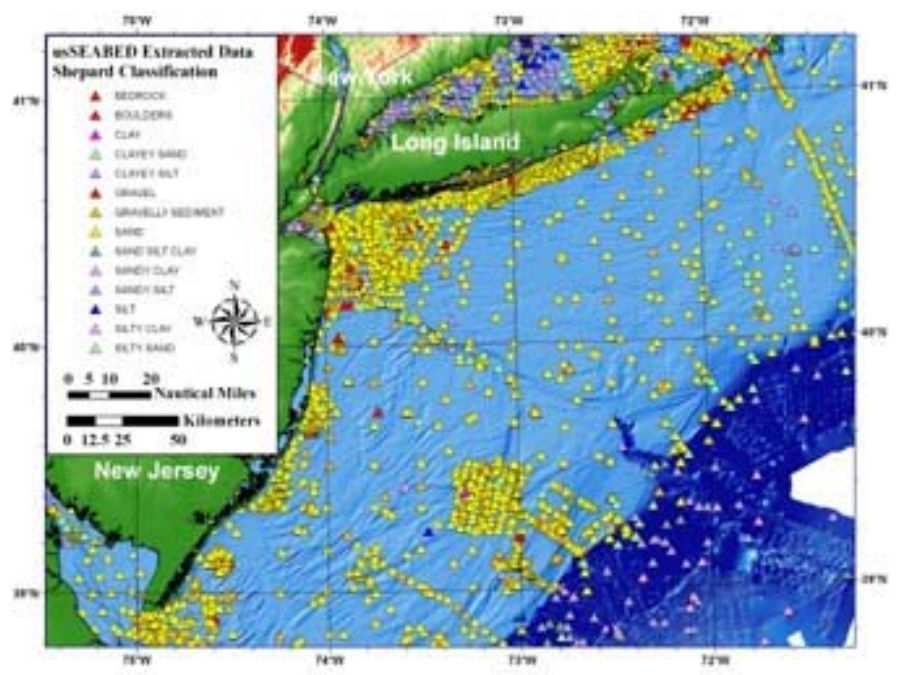

Map of Shepard sediment classification based on extracted (numeric) data only.

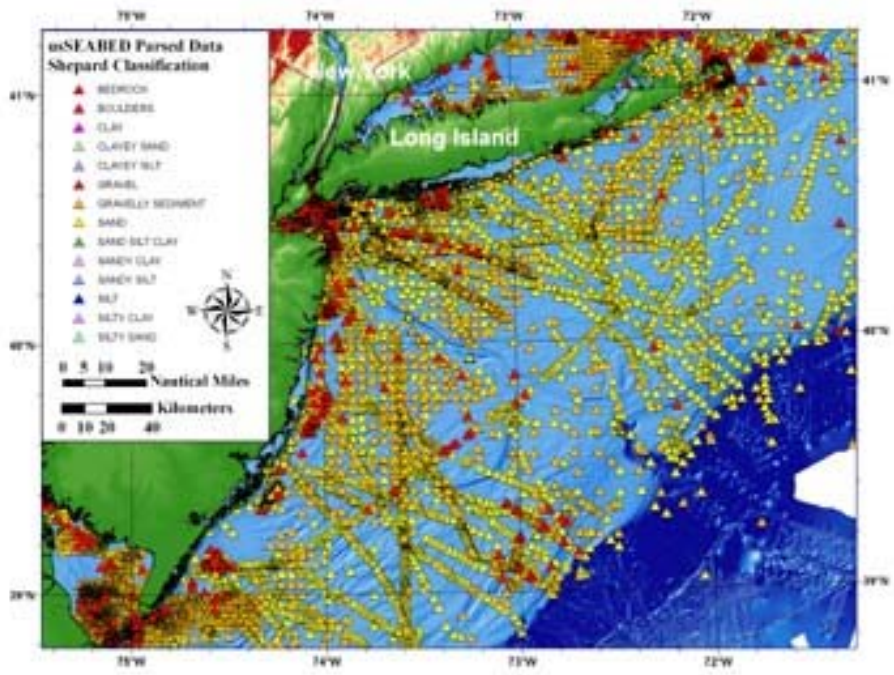

Map of Shepard sediment classification based on parsed (word-based) data only. The parsed dataset greatly increases the amount of available data , and overall geographical coverage. 


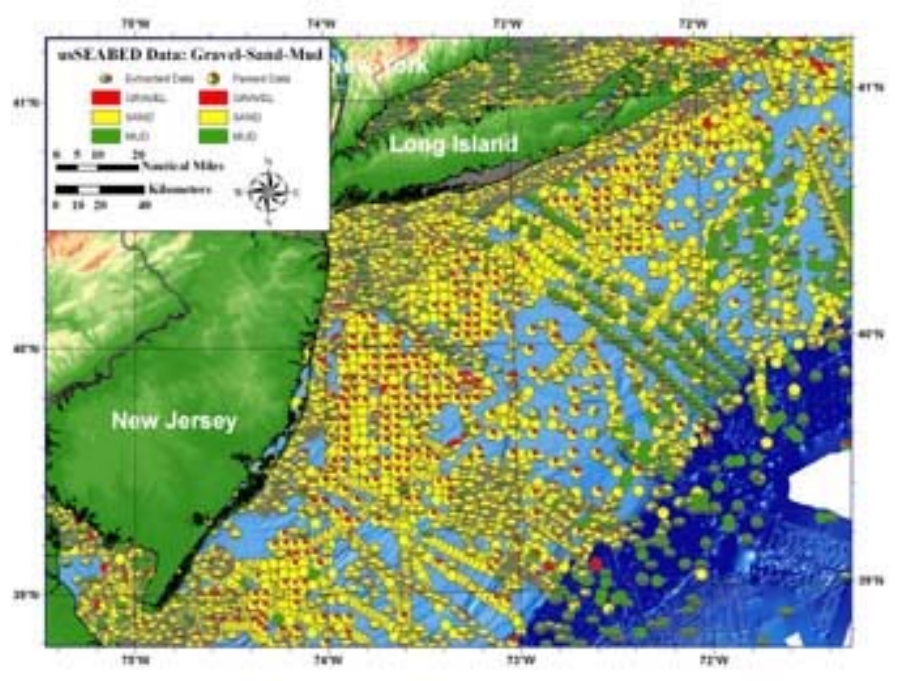

Map of gravel-sand-mud percentages. Map view symbols represent the parsed data, while oblique view symbols represent the extracted data. This type of a display offers more insight into the overall sediment composition than the simplified view of mean grain size. This is often the case for fish habitat studies, where sea floor composition is more important than generalized, or averaged, information.

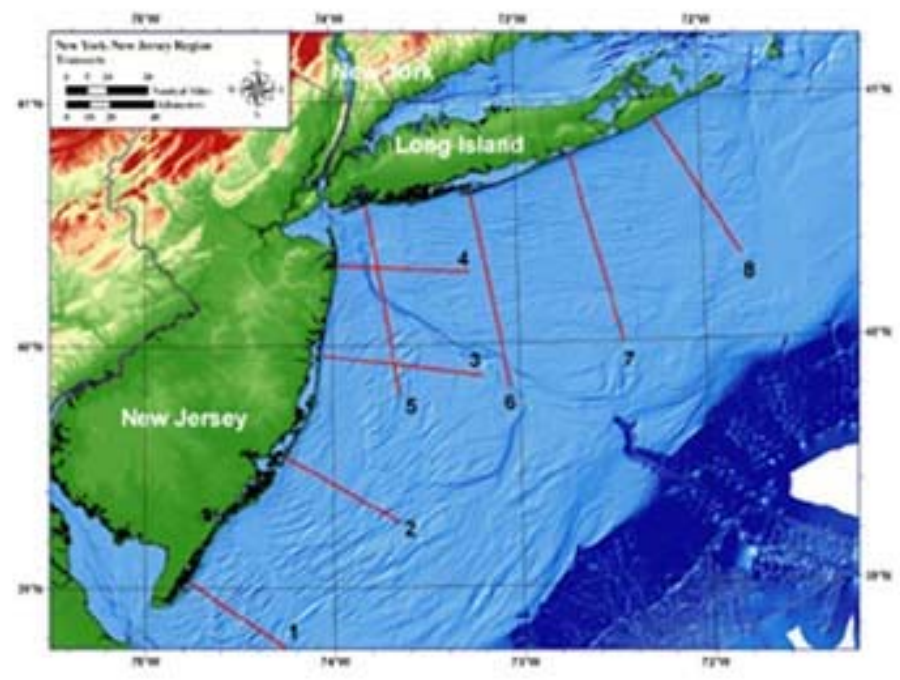

Example of shore-normal transects for Long Island and New Jersey, where usSEABED data may be examined superimposed on high resolution NOAA bathymetry. Click the image for a larger view, from which you will be able to click the line to bring up the transect details. 


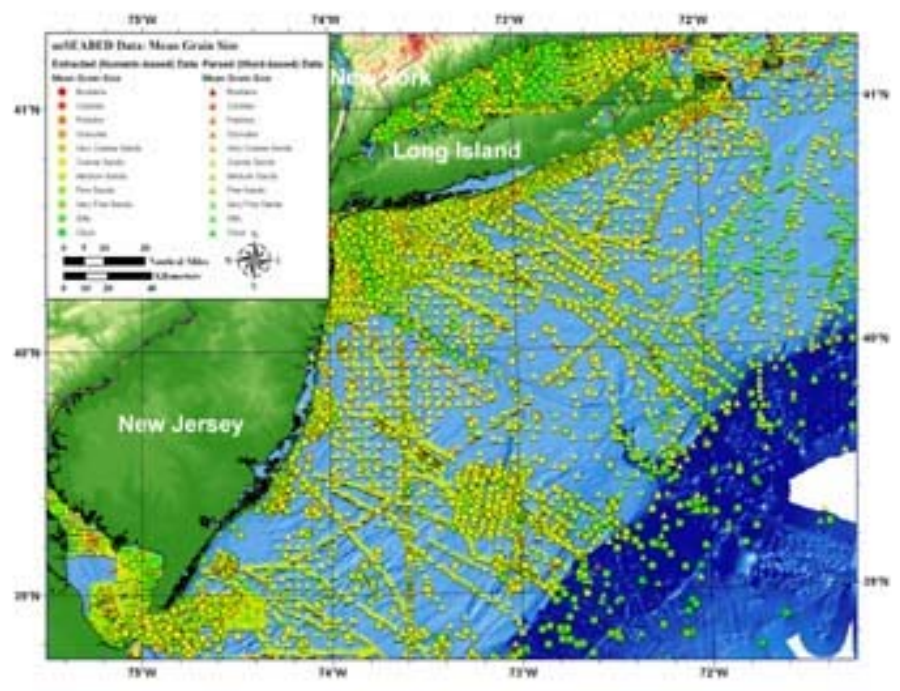

Map of mean grain size based on both extracted and parsed data in usSEABED. This is the same shown above, with the detail boxes in red.

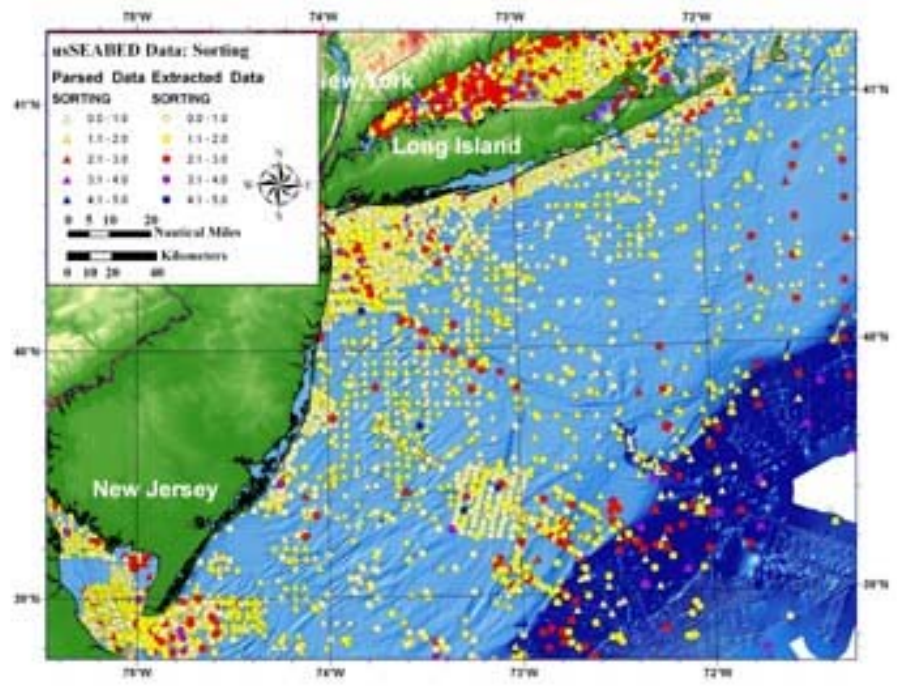

Map of sediment sorting of extracted and parsed data lover 29,000 samples for the New York-New Jersey region). 


\section{Selected References}

The following references contain basic textural data, discuss sea-floor sediment distribution, or relate to sediment classification schemes in the New York-New Jersey offshore region.

Allen-Lafayette, Z., Uptegrove, J., Waldner, J.S., Hall, D.W., Smith, P.C., Ashley, G.M., Sheridan, R.E., Goss, M.C., Muller, F.L., and Keller, E., 1998, Identified potential beach replenishment sand sources, onshore and offshore New Jersey, in Digital Mapping Techniques '98, Champaign, IL, 1998, Proceedings: Champaign, Ill., p. 104-105,

Alpine Geophysical Associates, 1969, Final report on data reduction for CERC New Jersey Offshore Sand Inventory Program: Coastal Engineering Research Center, 48 p.

Amato, R.V., 1994, Sand and gravel maps of the Atlantic Continental Shelf with explanatory text, U.S. Minerals Management Service OCS Monograph MMS 93-0037, scale 1:1,000,000, $35 \mathrm{p}$.

Amato, R.V., 1997, Management of sand resources on the continental shelf off New Jersey [abs.], in Geological Society of America, Northeastern Section, Annual Meeting, 32nd, King of Prussia, Penn., 1997, Proceedings: Abstracts with Programs, Geological Society of America, p. 27.

Anders, F.J., Bocamazo, L.M., Tanski, J., and Davies, D.S., 1995, A cooperative shoreline erosion monitoring program, Atlantic Coast of New York [abs.], in Coastal Zone '95, Tampa, Fla.: American Society of Civil Engineers, p. 496-497.

Baldwin, S.M., Mecray, E.L., Koopmans, D.J., and Buchholtz ten Brink, M.R., The distribution of sewage sludge, traced by elevated Silver and copper concentrations, along the Hudson Shelf Valley [abs.]: Geological Society of America, Northeastern and Southeastern Sections Joint Meeting, Tysons Corner, Va., March 25-27, 2004.

Bishop, K.J., 2000, Assessment of offshore sediments in Area C (offshore Barnegat Inlet) from the New Jersey Continental Shelf for potential use in beach replenishment: Lawrenceville, NJ, Rider University, Senior Thesis Project, 36 p.

Bocamazo, L.M., 1991, Sea Bright to Manasquan, New Jersey, beach erosion control projects: Shore \& Beach, v. 59, no. 3, p. 37-42.

Bokuniewicz, H.J., and Fray, C.T., 1979, The volume of sand and gravel resources in the lower bay of New York Harbor: Marine Sciences Research Center, State University of New York Special Report No. 32, 34 p.

Bokuniewicz, H.J., 1988, The study of subaqueous sand mining in New York Harbor: Marine Mining, v. 7, no. 1, p. 7-18.

Buchholtz ten Brink, M.R., Bothner, M.H., and Mecray, E.L., 2001, Contaminated coastal sediments in the northeastern United States; changing sources over time [abs.]: American Geophysical Union, Eos, Transactions, v. 82, no. 20, p. S230.

Buczkowski, B.J., Reid, J.A., Jenkins, C.J., Reid, J.M., Williams, S.J., and Flocks, J.G., 2006, usSEABED: Gulf of Mexico and Carribbean (Puerto Rico and U.S. Virgin Islands) Offshore Surficial Sediment Data Release: U.S. Geological Survey Data Series 146, Version 1.0, CDROM.

Butman, B., Noble, M., and Folger, D., 1979, Long term observations of bottom current and bottom sediment movement on the middle Atlantic continental shelf: Journal of Geophysical Research, v. 84, p. 1187-1205. 
Butman, B., Danforth, W.W., Schwab, W.C. and Buchholtz ten Brink, M.R.,1998, Multibeam bathymetric and backscatter maps of the Upper Hudson Shelf Valley and adjacent shelf, offshore of New York: U.S. Geological Survey Open-File Report 98-616, 4 sheets, scale $1: 125,000$.

Butman, B., 1998, Mapping the sea floor geology offshore of the New York - New Jersey metropolitan region: U.S. Geological Survey Fact Sheet, FS 133-98, 2 p.

Butman, B., Alexander, P.S., Harris, C.K., Traykovski, P.A., Buchholtz ten Brink , M.R., Lightsom, F.S., and Martini, M.A., 2003, Oceanographic observations in the Hudson Shelf Valley, December 1999 - April 2000: data report: U.S. Geological Survey Open-File Report 02-217, DVD-ROM.

Butman, B., Middleton, T.J., Thieler, E.R., and Schwab, W.C.,2003, Topography, shaded relief, and backscatter intensity of the Hudson Shelf Valley, offshore of New York: U.S. Geological Survey Open-File-Report 03-372, DVD-ROM.

Butman, B., Gutierrez, B.T., Buchholtz ten Brink, M.R., Schwab, W.C., Blackwood, D.S., and Middleton, T.J., 2003, Photographs of the sea floor offshore of New York and New Jersey: U.S. Geological Survey Open-File-Report 01-470, DVD-ROM.

Byrnes, M.R., Hammer, R.M., Vittor, B.A., Ramsey, J.S., Snyder, D.B., Wood, J.D., Bosma, K.F., Thibaut, T.D., and Philips, N.W., 2001, Environmental survey of potential sand resource sites: offshore New Jersey. Volume I: Main Text, Volume II: Appendices: U.S. Department of the Interior, Minerals Management Service, International Activities and Marine Minerals Division (INTERMAR), OCS Report MMS 2000-052, 664 p.

Carlisle, D., and Wallace, W.A., 1978, Sand and gravel in the greater New York area: what kind and how much?: New York Sea Grant Series, 68 p.

de Figueiredo, A.G., Jr., 1984, Submarine sand ridges: geology and development, New Jersey, USA: Coral Gables, Florida, University of Miami, Ph.D. Thesis, 524 p.

Duane, D.B., 1969, A study of New Jersey and northern New England coastal waters: Shore and Beach, v. 37, no. 2, p. 12 - 16.

Duane, D.B., 1969, Sand and gravel deposits in the nearshore continental shelf, Sandy Hook to Cape May, New Jersey [abs.]: Geological Society of America Meeting, Abstracts with Programs, v. Part 7, p. 53-54.

Emery, K.O., and Milliman, J.D., 1979, Quaternary sediments of the Atlantic continental shelf off the United States: Quaternaria, v. 12, p. 3-18.

Emery, K.O., and Uchupi, E., 1984, The Geology of the Atlantic Ocean: New York, NY, Springer-Verlag, $1050 \mathrm{p}$.

Esker, D., 1993, Synthetic seismograms from vibracores; a tool for correlating the seismic record to the sediment record of Barnegat Inlet, New Jersey: New Brunswick, NJ, Rutgers, The State University, New Brunswick, Master's Thesis, 198 p.

Esker, D., Sheridan, R.E., Ashley, G.M., Waldner, J.S., and Hall, D.W., 1996, Synthetic seismograms from vibracores; a case study in correlating the late Quaternary seismic stratigraphy of the New Jersey inner continental shelf: Journal of Sedimentary Research, v. 66, no. 6, p. 1156-1168.

Folk, R.L., 1954. The distinction between grain size and mineral composition in sedimentary rock nomenclature. Journal of Geology 62 (4), 344-359.

Folk, R.L., 1974, The petrology of sedimentary rocks: Austin, Tex., Hemphill Publishing Co., $182 \mathrm{p}$.

Foster, D.S., Swift, B.A. and Schwab, W.C., 1999, Stratigraphic Framework Maps of the Nearshore Area of Southern Long Island from Fire Island to Montauk Point, New York: U.S. Geological Survey Open-File Report 99-559. 
Freeland, G.L., and Swift, D.J.P., 1978, MESA New York Bight Atlas Monograph 10: New York Sea Grant Institute, 93 p.

Garrison, L.E., and McMaster, R.L., 1966, Sediments and geomorphology of the continental shelf off southern New England: Marine Geology, v. 4, p. 273-289.

Gaswirth, S.B., 1999, The late Pleistocene to Holocene glacial history of Raritan Bay, New Jersey: New Brunswick, NJ, Rutgers, The State University of New Jersey, New Brunswick, M.S. Thesis, 157

Harris, C.K., Butman, B., and Traykovski, P., 2003, Winter-time circulation and sediment transport in the Hudson Shelf Valley: Continental Shelf Research, v. 23, no. 8.

Hastings M.E., Poppe, L.J., and Hathaway, J.C., 2000, Surficial sediment database, in: Poppe, L.J., and Polloni, C.F. (eds.) USGS East-Coast Sediment Analysis: Procedures, Database, and Georeferenced Displays, U.S. Geological Survey Open-File Report 00-358, CD-ROM.

Hathaway, J.C., 1971, Data File-Continental Margin Program, Atlantic Coast of the United States: Woods Hole Oceanographic Institution Reference No. 71-15, 496 p.

Kastens, K.A., Fray, C.T., and Schubel, J.R., 1978, Environmental effects of sand mining in the lower bay of New York Harbor; phase 1: Marine Sciences Research Center, State University of New York Special Report no. 15, 139 p.

Knebel, H.J., Signell, R.P., Rendigs, R.R., Poppe, L.J., and List, J.H., 1998, Maps and illustrations showing the acoustic and textural characteristics and the distribution of bottom sedimentary environments, Long Island Sound, Connecticut-New York in Long Island Sound Environmental Studies (L.J. Poppe and C. Polloni, editors): U.S. Geological Survey Open-File Report 98-502, Chapter 1, CD-ROM, 14 p.

Knebel, H.J., Signell, R.P., Rendigs, R.R., Poppe, L.J., and List, J.H., 1999, Sea-floor environments in the Long Island Sound estuarine system: Marine Geology, v. 155, no. 3-4, p. 277-318.

Knebel, H.J., Lewis, R.S., and Varekamp, J.C., 2000, Regional processes, conditions, and characteristics of the Long Island Sound sea floor: Journal of Coastal Research, v. 16, no. 3, p. 519-521.

Knebel, H.J., and Poppe, L.J., 2000, Sea-floor environments within Long Island sound--A regional overview: Journal of Coastal Research, Special Thematic Section, v. 16, no. 3, p. 535-550.

Lewis, R.S., and Neff, N.F., 1992, Continental margins program - years five and six: stratigraphic and depositional history of Long Island Sound [abs.]: Program and abstracts from the Third Symposium on Studies Related to Continental Margins: a summary of year-five and year-six Activities, p. 32.

Lewis, R.S., Robinson, A.C., McHone, N., DiGiacomo-Cohen, M.L., Poppe, L.J., and Polloni, C., 1998, Long Island Sound bibliographic database, in Poppe, L.J., and Polloni, C., eds., Long Island Sound Environmental Studies: U.S. Geological Survey Open-File Report 98-502, Chapter 7, CD-ROM.

Lubchansky, B.J., 1999, Stratigraphic relationships of nine core samples collected from offshore shoals located in federal waters off Atlantic City, New Jersey, in 1999 Assateague Shelf and Shore Workshop, 25th: Lewes, Del., University of Delaware, unpaginated.

Lubchansky, B.J., 1999, Stratigraphic relationships of nine core samples collected from offshore shoals located in federal waters off Atlantic City, New Jersey: Lawrenceville, NJ, Rider University, Senior Thesis Project, with appendix.

Marine Resources Development Corporation, 1979, The feasibility of offshore mining of construction minerals in the Greater New York Metropolitan Area: A technical survey: Report to U.S. Geological Survey by MRDC, $131 \mathrm{p}$. 
Mauriello, M.N., 1991, Beach nourishment and dredging; New Jersey's policies: Shore \& Beach, v. 59, no. 3, p. 25-28.

Mecray, E.L., Buchholtz ten Brink, M.R. and Butman, B., 1999, Contaminants and marine geology in the New York Bight: modern sediment dynamics and a legacy for the future: U.S. Geological Survey Fact Sheet FS-114-99, May 1999.

Mecray, E.L., Reid, J.M., Hastings, M.E., and Buchholtz ten Brink, M.R., 2003, Contaminated Sediments Database for Long Island Sound and the New York Bight : U.S. Geological Survey Open-File Report 03-241.

McKinney, T.F., and Friedmann, G.M., 1970, Continental shelf sediments of Long Island, New York: Journal of Sedimentary Petrology, v. 40, no. 1, p. 213-248.

Meisburger, E.P., and Williams, S.J., 1980, Sand resources on the inner Continental Shelf of the Cape May region, New Jersey: U.S. Army Corps of Engineers Coastal Engineering Research Center Miscellaneous Report MR 80-4, 40 p.

Meisburger, E.P., and Williams, S.J., 1981, Use of vibratory coring samples for sediment surveys: U.S. Army Corps of Engineers Coastal Engineering Research Center Technical Aid 81-9, 18 p.

Meisburger, E.P., and Williams, S.J., 1982, Sand resources on the inner continental shelf off the central New Jersey coast: U.S. Army Corps of Engineers Coastal Engineering Research Center Miscellaneous Report MR 82-10, 48 p.

Milliman, J.D., 1972, Atlantic continental shelf and slope of the United States; petrology of the sand fraction of sediments, northern New Jersey to southern Florida: U.S. Geological Survey Professional Paper 529-J, p. J1 - J40.

Muessig, K.W., Uptegrove, J., Waldner, J.S., Mullikin, L.G., Hall, D.W., Gilroy, J., and Muller, F.J., 1994, New Jersey's cooperative study of offshore sand resources in Federal waters for beach replenishment [abs.]: Geologic Society of America Abstracts with Programs, v. 26, no. 3, p. 64.

Mugler, M.W., 1981, Beach nourishment with dredged material: U.S. Army Engineer Institute for Water Resources Policy Study 81-0110, 66 p.

Muller, F.L., Uptegrove, J., Miller, K.G., Aubry, M.-P., Browning, J.V., Bukry, D., Burckle, L.D., Feigenson, M.D., Goss, M., Gwynn, D., Heibel, T., Kent, D.V., Liu, C., Mullikin, L., Pekar, S.F., Powars, D., Queen, D., Sugarman, P.J., and Van Fossen, M.C., 1997, Data report; Heavy mineral analysis of the upper Miocene(?) to Pleistocene sands, Cape May site, in Ocean Drilling Program, Scientific Results; New Jersey coastal plain; covering onshore boreholes as part of the New Jersey sea-level transect, Island Beach and Atlantic City sites, March-August 1993, Cape May site, March-April 1994: Texas A \& M University, Ocean Drilling Program, College Station, Tex., United States, p. 75-79.

Nevin, C.M., 1929, Sand and gravel resources of New York State: New York Geological Survey Bulletin 282, $180 \mathrm{p}$.

New Jersey Department of Environmental Protection, and U.S. Army Corps of Engineers, 2001, Manasquan Inlet to Barnegat Inlet feasibility study: New Jersey Department of Environmental Protection, U.S. Army Corps of Engineers Draft Feasibility Report, Integrated Environmental Impact Statement 01-679.

Ocean/Seismic/Survey, 1981, Identification of potential ocean borrow sources offshore of Fire Island Inlet to Montauk Point, New York: New York District Corps of Engineers Contract No. DACW51-80-C-0034, 55 p.

Paskevich, V.F., Poppe, L.J., Hastings, M.E., and Hathaway, J.C., 2001, Sea-floor photography from the Continental Margin program - a pictorial survey of benthic character and habitats along the U.S. east coast: U.S. Geological Survey Open-File Report 01-154, CD-ROM. 
Poppe, L.J., and Polloni, C., 1998, Long Island Sound environmental studies: U.S. Geological Survey Open-File Report 98-502, CD-ROM.

Poppe, L.J., and Polloni, C.F. (eds.), 2000, USGS East-Coast Sediment Analysis: Procedures, Database, and Georeferenced Displays, U.S. Geological Survey Open-File Report 00-358, CD-ROM.

Poppe, L.J., Williams, S.J., and Paskevich, V.F., Editors, (2005). "USGS East-coast sediment analysis: procedures, database, and GIS data", U.S. Geological Survey Open-File Report 2005-1001, DVD-ROM.

Pratt, R.M., 1968, Atlantic continental shelf and slope of the United States: physiography and sediments of the deep-sea basin: U.S. Geological Survey Professional Paper 529-B, 44 p.

Pulliam, J., Austin, J.A., Jr., Luhurbudi, E.C., Saustrup, S., and Stoffa, P.L., 1996, An ultrahigh resolution 3-D survey of the shallow subsurface on the continental shelf of New Jersey: Leading Edge, v. 15, no. 7, p. 839-845.

Reid, J.M., Reid, J.A., Jenkins, C.J., Hastings, M.E., Williams, S.J., and Poppe, L.J. 2005. "usSEABED: Atlantic coast offshore surficial sediment data release", U.S. Geological Survey Data Series 118, Version 1.0, CD-ROM.

Reid, J.A., Reid, J.M., Jenkins, C.J., Zimmermann, M., Williams, S.J., and Field, M.E., 2006, usSEABED: Pacific Coast (California, Oregon, Washington) Offshore Surficial-Sediment Data Release: U.S. Geological Survey Data Series 182, version 1.0.

Richards, H.G., 1983, Generalized structural contour maps of the New Jersey Coastal Plain: New Jersey Geological Survey Report 4, 38 p.

Rine, J.M., Tillman, R.W., and Swift, D.J.P., 1986, Generation of late Holocene sand ridges on the middle continental shelf off New Jersey, U.S.A. [abs.], in SEPM Annual Midyear

Meeting, Raleigh, N.C.: Society of Economic Paleontologists and Mineralogists, p. 95.

Rock Color Chart Committee, reprinted 1991, Rock Color Chart: Boulder, Colo., Geological Society of America, size 5 1/8" by 7 1/2".

Ross, D.A., 1970, Atlantic continental shelf and slope of the United States: heavy minerals of the continental margin from southern Nova Scotia to northern New Jersey: U.S. Geological Survey Professional Paper 529-G, 40 p.

Schlee, J., 1968, Sand and gravel on the continental shelf off the northeastern United States: U.S. Geological Survey Circular 602, 9 p.

Schlee, John, 1973, Atlantic Continental Shelf and Slope of the United States sediment texture of the northeastern part: U.S. Geological Survey Professional Paper 529-L, 64 p.

Schlee, J., (with a section by Sanko, P.), 1975, Sand and gravel: New York Sea Grant Institute MESA New York Bight Atlas Monograph 21, 26 p.

Schlee, J.S., 1964, New Jersey offshore gravel deposit: Pit and Quarry, v. 57, no. 6, p. 80-81, 95. Schwab, W.C., Allison, M.A., Corso, W., Lotto, L.L., Butman, B., Buchholtz ten Brink, M., Denny, J., Danforth, W.W., and Foster, D.S., 1997, Initial results of high-resolution sea-floor mapping offshore of the New York New Jersey metropolitan area using sidescan sonar: Northeastern Geology and Environmental Sciences, v. 19, no. 4, p. 243-262.

Schwab, W.C., Thieler, E.R., Allen, J.R., Foster, D.S., Swift, B.A., Denny, J.F., and Danforth, W.W., 1999, Geologic Mapping of the nearshore area offshore Fire Island, New York, in Kraus, N.C., and McDougal, W.G., eds.: Coastal Sediments '99, v. 2, p. 1552-1567.

Schwab, W.C., Denny, J.F., Butman, B., Danforth, W.W., Foster, D.S., Swift, B.A., Lotto, L.L., Allison, M.A. and Thieler, E.R., 2000, Sea-floor characterization offshore of the New YorkNew Jersey metropolitan area using sidescan sonar: U.S. Geological Survey Open-File Report 00-295. 
Schwab, W.C., Thieler, E.R., Denny, J.F., Danforth, W.W. and Hill, J.C., 2000, sea floor Sediment Distribution Off Southern Long Island, New York: U.S. Geological Survey Report 00-243.

Schwab, W.C., Thieler, E.R., Allen, J.R., Foster, D.S., Swift, B.A., and Denny, J.F., 2000, Influence of inner-continental shelf geologic framework on the evolution and behavior of the barrier-island system between Fire Island Inlet and Shinnecock Inlet, Long Island, New York: Journal of Coastal Research, v. 16, n. 2, p. 408-422.

Schwab, W.C., Denny, J.F., Foster, D.S., Lotto, L.L., Allison, A.L., Uchupi, E., Swift, B.A., Danforth, W.W., Thieler, E.R., and Butman, B., 2003, High-resolution quaternary seismic stratigraphy of the New York Bight Continental Shelf: U.S. Geological Survey Open-File Report 02-152, DVD-ROM.

Shepard, F.P., 1954, Nomenclature based on sand-silt-clay ratios: Journal Sedimentary Petrology, v. 24, p. 151-158.

Sheridan, R.E., Ashley, G.M., Miller, K.G., Waldner, J.S., Hall, D.W., and Uptegrove, J., 2000, Offshore-onshore correlation of upper Pleistocene strata, New Jersey Coastal Plain to continental shelf and slope: Sedimentary Geology, v. 134, no. 1-2, p. 197-207.

Signell, R.P., Knebel, H.J., List, J.H., and Farris, A., 1998, Physical processes affecting sedimentary environments in Long Island Sound: U.S. Geological Survey Open-File Report 98-502, Chapter 6, CD-ROM, 10 .

Smith, P.C., 1996, Nearshore ridges and underlying Pleistocene sediment on the inner continental shelf of New Jersey: New Brunswick, NJ, The State University of New Jersey, M.S. Thesis, 55 p.

Smith, S.J., Gravens, M.B., and Smith, J.M., 1999, Monmouth Beach, New Jersey: beach-fill "hot spot" erosion evaluation. Report 1: physical processes analysis: U.S. Army Corps of Engineers, New York District Technical Report CHL-99-13, 91 p.

Smith, S.J., Gravens, M.B., and Smith, J.M., 1999, Monmouth Beach, New Jersey: beach-fill "hot spot" erosion evaluation. Report 2: Functional design of shore protection alternatives for beach-fill longetivity: U.S. Army Corps of Engineers, New York District Technical Report CHL-99-13, 77 p.

Snedden, J.W., Tillman, R.W., Kreisa, R.D., Schweller, W.J., Culver, S.J., and Winn, R.D., Jr., 1994, Stratigraphy and genesis of amodern shoreface-attached sand ridge, Peahala Ridge, New Jersey: Journal of Sedimentary Research, v. B64, no. 4, p. 560 - 581.

Squires, D.F., 1988, Evolution of New York state's policies on underwater mining of sand and gravel: Marine Sand and Gravel Mining, v. 7, no. 1/2, p. 19 - 34.

Stetson, H.C., 1936, Geology and paleontology of the Georges Bank canyons: Bulletin Geological Society of America, v. 47, p. 339-366.

Stetson, H.C., 1938, The sediments of the continental shelf off the eastern coast of the United States: Massachusetts Institute of Technology and Woods Hole Oceanographic Institution, Papers in Physical Oceanography and Meteorology, v.5, no. 4, 48 p.

Stubblefield, W.L., and Swift, D.J.P., 1981, Grain size variation across sand ridges, New Jersey continental shelf: Geo-Marine Letters, v. 1, no. 1, p. 45 - 48.

Stubblefield, W.L., McGrail, D.W., and Kersey, D.G., 1984, Recognition of transgressive and post-transgressive sand ridges on the New Jersey continentel shelf, in Tillman, R.W., and Siemers, C., eds., Siliclastic Shelf Sediments: Tulsa, OK, Society of Economic Paleontologists and Mineralogists (Society for Sedimentary Geology), v. 34, p. 1-23.

Swift, D.J.P., Moir, R., and Freeland, G.L., 1980, Quaternary rivers on the New Jersey shelf: relation of sea floor to buried valleys: Geology, v. 8, no. 6, p. 276-280. 
Swift, D.J.P., McKinney, T.F., and Stahl, L., 1984, Recognition of transgressive and posttransgressive sand ridges on the New Jersey continental shelf: discussion, in Tillman, R.W., and Siemers, C.T., eds., Siliciclastic shelf sediments: Tulsa, OK, Society of Economic Paleontologists and Mineralogists (Society for Sedimentary Geology), p. 25-36.

Trumbull, J., 1972, Atlantic continental shelf and slope of the United States: sand-size fraction of bottom sediments, New Jersey to Nova Scotia: U.S. Geological Survey Professional Paper 529-K, p. K1 - K45.

U.S. Mineral Management Service, 1999, Environmental Report: use of federal offshore sand resources for beach and coastal restoration in New Jersey, Maryland, Delaware, and Virginia OSC study MMS 99-0036, 1 CD-ROM.

Uptegrove, J., Grosz, A., Maharaj, S.V., and Muller, F.J., 1991, Physiographic, bathymetric and textural controls on heavy-mineral enrichment in surficial sediments of the New Jersey shelf [abs.]: Geologic Society of America Abstracts with Programs, v. 23, no. 1, p. 142.

Uptegrove, J., Grosz, A., Maharaj, S.V., Muller, F.J., Muessig, K.W., Farnsworth, J., Burbanck, G.P., and Cheung, T.T., 1991, Preliminary textural and mineralogic analyses of vibracore samples collected between Absecon and Barnegat Inlets, New Jersey: New Jersey Geological Survey Open-File Report 91-3, 11 p.

Uptegrove, J., Muessig, K.W., Muller, F.L., Maharaj, S.V., and Grosz, A.E., 1992, Distribution of heavy minerals and gravel in sediments of the New Jersey Shelf as determined from grab and vibracore samples [abs.], in Symposium on Studies Related to Continental Margins, 3rd, p. 30.

Uptegrove, J., Muller, F.J., Maharaj, S.V., Grosz, A., and Muessig, K.W., 1992, Distribution of heavy-minerals and gravel in offshore sediments as determined from vibracore samles from Barnegat to Absecon Inlets, New Jersey: Abstracts with programs, Northeastern Section, Geological Society of America, Harrisburg, PA, p. 81.

Uptegrove, J., Muessig, K.W., Grosz, A., Muller, F.J., and Maharaj, S.V., 1994, Distribution of heavy minerals and gravel in sediments of the New Jersey shelf as determined from grab and vibracore samples, in Symposium on Studies Related to Continental Margins, 3rd: Austin, Twx., Minerals Management Service and Association of American State Geologists, p. 106112.

Uptegrove, J., Mullikin, L.G., Waldner, J.S., Ashley, G.M., Sheridan, R.E., Hall, D.W., Gilroy, J., and Farrell, S.C., 1995, Characterization of offshore sediments in federal waters as potential sources of beach replenishment sand; Phase I: Minerals Management Service, U.S. Department of the Interior Cooperative Agreement \#14-35-0001-30666, New Jersey Geological Survey Open-File Report 95-1, 150 p.

Uptegrove, J., Waldner, J.S., Smith, P.C., Mullikin, L.G., Sheridan, R.E., Ashley, G.M., Hall, D.W., and Farrell, S.C., 1996, Identification of shoreline erosion areas and characterization of beach-replenishment sand sources for New Jersey, in Annual Meeting of the Association of Engineering Geologists, 39th: East Brunswick, N.J., p. 71.

Uptegrove, J., Waldner, J.S., Hall, D.W., Smith, P.C., Ashley, G.M., Sheridan, R.E., AllenLafayette, Z., Goss, M.C., Mueller, F.L., and Keller, E., 1997, Characterization of sediments in federal waters offshore of New Jersey as potential sources for beach replenishment sand; Phase II Year 2 Final Report: U.S. Minerals Managament Service Cooperative Agreement \#14-35-0001-30751, 29 p.

Uptegrove, J., Hall, D.W., Waldner, J.S., Sheridan, R.E., Lubchansky, B.J., and Ashley, G.M., 1999, Geologic framework of the New Jersey inner shelf: results from resource-based seismic and vibracore studies, in Puffer, J.H., ed., New Jersey beaches and coastal processes from a 
geologic and environmental perspectives; Newark, N.J., Geological Association of New Jersey, p. 45 - 64.

Uptegrove, J., Hall, D.W., Waldner, J.S., Lubchansky, B.J., Sheridan, R.E., and Ashley, G.M., 2000, Pleistocene/Holocene geology of the New Jersey Inner Shelf: results from resourcebased seismic and vibracore studies [abs.]: Geologic Society of America Abstracts with Programs, v. 32, no. 1, p. 80.

Vincent, C.E., Swift, D.J.P., and Hillard, B., 1981, Transport in the New York bight, North American Atlantic shelf: Marine Geology, v. 42, no. 1-4, p. 369-398.

Waldner, J.S., and Others, 1991, A marine seismic survey to delineate Tertiary and Quaternary stratigraphy of coastal plain sediments offshore to Atlantic City, New Jersey: New Jersey Geological Survey Report 26, 15 p.

Waldner, J.S., Hall, D.W., Uptegrove, J., Sheridan, R.E., and Ashley, G.M., 1996, Geophysical search for offshore borrow-pit sites to dispose of dredge spoils in New York-New Jersey Harbor, in Association of Engineering Geologists Annual Meeting, 39th, East Brunswick, NJ, Association of Engineering Geologists, p. 72.

Waldner, J.S., Hall, D.W., Uptegrove, J., Sheridan, R.E., Ashley, G.M., and Esker, D., 1999, Assessment of offshore New Jersey sources of beach replenishment sand by diversified application of geologic and geophysical methods [abs.]: Marine Georesources and Geotechnology, v. 17, no. 2-3, p. 139-140.

Waldner, J.S., Sheridan, R.E., Uptegrove, J., Hall, D.W., and Ashley, G.M., 1999, Prospecting for sand, offshore New Jersey, in International Symposium on Coastal Engineering and Science of Coastal Sediment Processes, 4th: Hauppauge, N.Y., American Society of Civil Engineers, p. 2090-2101.

Wentworth, C.K., 1922, A scale of grade and class terms for clastic sediments: Journal of Geology, v. 30, p. 377-392.

Wentworth, C.K., 1929, Method of computing mechanical composition of sediments: Geological Society of America Bulletin, v. 40, p. 771-790.

Wellner, R.W., 1990, High-resolution seismic stratigraphy and depositional history of Barnegat Inlet, New Jersey and vicinity: New Brunswick, New Jersey, The State University of New Jersey, M.S. Thesis, 109 p.

Williams, S.J., and Field, M.E., 1971, Sediments and shallow structures of the inner continental shelf off Sandy Hook, New Jersey [abs.]: Geological Society of America Abstracts with Programs, v. 3, no. 1, p. 62.

Williams, S.J., 1973, The geologic framework of inner New York Bight - its influence on positioning offshore engineering structures [abs.]: Geological Society of America Abstracts with Programs, v. 5, no. 2, p. 239.

Williams, S.J., and Duane, D.B., 1974, Geomorphology and sediments of the inner New York Bight continental shelf: U.S. Army, Corps of Engineers, Coastal Engineering Research Center, TM-45, $81 \mathrm{p}$.

Williams, S.J., 1976, Geomorphology, shallow subbottom structure, and sediments of the Atlantic Inner Continental Shelf off Long Island, New York: Dept. of the Army, Coastal Engineering Research Center, Technical Paper TP 76-2, 123 p.

Williams, S.J., 1976, Geologic structure, stratigraphy, and sediment distribution on the inner continental shelf off Long Island, New York [abs.]: Geological Society of America Abstracts with Programs, v. 8, no. 2, p. 301.

Williams, S.J., 1981, Sand resources and geological character of Long Island Sound: U.S. Army Corps of Engineers Coastal Engineering Research Center, Technical Paper TP 81-3, 65 p. 
Williams, S.J., and Meisburger, E.P., 1987, Sand sources for the transgressive barrier coast of Long Island, New York: evidence for landward transport of shelf sediments, in Specialty Conference on Advances in Understanding of Coastal Sediment Processes: Coastal Sediments '87, New Orleans, La., American Society of Civil Engineers, p. 1517-1532.

Williams, S.J., 1992, Sand and gravel - an enormous offshore resource within the U.S. Exclusive

Economic Zone, in DeYoung, J.H., Jr., and Hammarstrom, J.M., eds., Contributions to Commodity Geology Research: U.S. Geological Survey Bulletin 1877, p. H1-H10.

Williams, S. J., Jenkins, C., Currence, J., Penland, S., Reid, J., Flocks, J., Kindinger, J., Poppe, L., Kulp, M., Manheim, F., Hampton, M., Polloni, C., and Rowland, J. (2003). "New digital geological maps of U.S. continental margins: insights to sea floor sedimentary character, aggregate resources and processes, Proceedings of the International Conference on Coastal Sediments 2003,: Corpus Christi, Tex., World Scientific Publishing Corporation and East Meets West Productions, Corpus Christi, Tex., 11 p., CD-ROM.

Williams, S.J., Reid, J., and Manheim, F. (2004). "A Bibliography of Selected References to U.S. Marine Sand and Gravel Mineral Resources", U.S. Geological Survey Open-File Report 03-300.

Wolff, F.P., 1994, Evidence of the effects of onshore sand migration from the shoreface across the barrier islands of the south shore of Long Island, New York [abs.]: Geological Society of America Abstracts with Programs, v. 26, no. 7, p. 178-179.

\section{Related Websites}

This report is a product of The USGS Marine Aggregate Resources and Processes Project, and is the first of a series of publications directed at promoting a better understanding of sea floor composition, texture, and character, through use of the usSEABED data series.

The U.S. Geological Survey data and data products included in this open-file report have come from a collection of individuals representing their work and their research groups. Some of these groups and research projects can be found at the links below.

\section{USGS Links}

- USGS Woods Hole Science Center

- USGS Minerals Resources Program

- usSEABED Home

- USGS Center for Coastal and Watershed Studies

- USGS Pacific Science Center

- USGS Studies in the New York Bight

The collaborators listed below have contributed to the usSEABED dataset, and continue to be involved in the future developments of the project.

\section{Our Collaborators}

- U.S. Army Corps of Engineers

- NOAA National Marine Fisheries Service

- NOAA National Ocean Service

- Minerals Management Service

- U.S. Environmental Protection Agency 
- New Jersey Geological Survey

- New York State Geological Survey

- dbSEABED at INSTAAR/University of Colorado

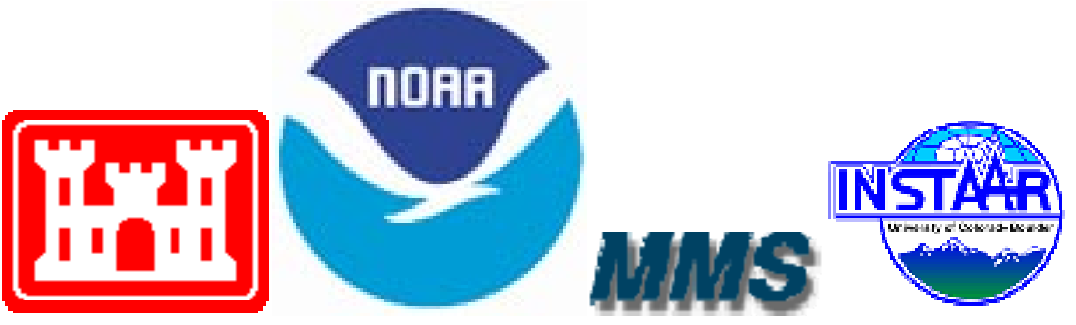

\section{Acknowledgments}

We thank Bradford Butman (USGS) and Ellen Mecray (formerly USGS, now NOAA) for critically reviewing this report and offering suggestions. Special acknowledgment is given to our colleague, Frank Manheim (USGS retired) for his leadership and hard work developing marine sediment data bases over much of his distinguished career, and particularly for his work in the New York Bight region.

\section{Contacts}

For information about the Marine Aggregates project:

S. Jeffress Williams, jwilliams@ usgs.gov, U.S. Geological Survey, Woods Hole Science Center, 384 Woods Hole Rd, Woods Hole, MA 02543-1598

\section{To contribute Atlantic Coast and/or Gulf Coast data:}

Matthew A. Arsenault, U.S. Geological Survey, Woods Hole Science Center, 384 Woods Hole Rd, Woods Hole, MA 02543-1598, 508-547-8700.

For questions about sediment data collection; laboratory and analysis techniques: Lawrence J. Poppe, lpoppe @usgs.gov, U.S. Geological Survey, Woods Hole Science Center, 384 Woods Hole Rd, Woods Hole, MA 02543-1598

For information about the Benthic Habitats project and/or adding Pacific Coast, Alaska or Hawaii data:

Jane A. Reid, jareid@usgs.gov,U.S. Geological Survey, Western Coastal and Marine Geology, Pacific Science Center, 400 Natural Bridges Drive, Santa Cruz, CA 95060

For questions about the dbSEABED program as well as global data:

Chris Jenkins, chris.jenkins@ @ colorado.edu, Institute of Arctic and Alpine Research, University of Colorado at Boulder, 1560 30th Street, Campus Box 450, Boulder, CO 80309-0450 


\section{For Educators}

The U.S. Geological Survey provides scientific information intended to help educate the public about natural resources, natural hazards, geospatial data, and issues that affect our quality of life. The USGS serves the Nation by providing reliable scientific information to describe and understand the Earth; and enhance and protect our quality of life.

The materials and Web sites below represent the combined efforts of the USGS and our collaborating institutions to reach the ever growing needs of the public. The products and Web links below are to serve as a starting point for educators to see what types of materials are available to assist in lesson planning, public presentations, and increasing the scientific understanding of our dynamic planet.

Animated Diagrams: Animated plots from a USGS study of contaminated sediments in the Hudson Shelf Valley as part of a presentation: Baldwin, S.M., Mecray, E.L., Koopmans, D.J., and Buchholtz ten Brink, M.R., The distribution of sewage sludge, traced by elevated Silver and copper concentrations, along the Hudson Shelf Valley [abs.]: Geological Society of America, Northeastern and Southeastern Sections Joint Meeting, Tysons Corner, Va. , March 25-27, 2004. (Silver concentrations in sediments )(Copper concentrations in sediments )

Presentation: A presentation summarizing results of the Marine Aggregate Resources and Processes project as part of the paper: Williams, S.J., 2005, Regional-scale understanding of the geologic character and sand resources of the Atlantic inner continental shelf, Maine to Virginia, Proceedings, Second Regional Conference on Dredging, Beach Nourishment, and Bird Conservation, 25-27 Oct 2005 (presentation).

References: References on marine sand and gravel studies and resources can be found in Williams and others (2003) A bibliography of selected references to U.S. Marine Sand and Gravel Resources, USGS Open-File Report 03-300 (online) and can help provide background information on the geologic framework of continental shelf sediments and processes.

Plotting: Plotting sediment data from the usSEABED program can be done using the following Matlab plot routines: (shepard.m) and (schlee.m). These scripts were written by members of the Woods Hole Science Center staff to help visualize the compositional components of sediment samples on a ternary diagram. They are available as part of the user contributed code library on the Mathworks Web site.

\section{Additional Resources Available Online:}

USGS maintains a Web page of Educational Resources, (including A Primer On Natural Aggregate, and Schoolyard Geology).

USGS Coastal and Marine Geology Program provides additional information and educational resources that are available on their Web site, searchable by the term 'Educational Materials'.

The NOAA Coastal Services Center is an office within the National Oceanic and Atmospheric Administration devoted to serving the nation's state and local coastal resource management programs.

Minerals Management Service maintains a Kids' Pages with excellent materials on a number of scientific topics, including The Ocean's Sand, A Natural Resource (Adobe pdf format) 
U.S. Army Corps of Engineers has an Education Center which includes lessons and materials in navigation, sedimentation, and many other topics tied to engineering in the coastal environment.

New York State GIS Clearinghouse has an Education Outreach Web page that includes training events, a who's who contact list, and other resources.

New Jersey Department of Environmental Protection GIS Website has a presentations Web page where power-point presentations can be downloaded.

\section{Presentation}

\section{Regional-scale understanding of the geologic character and sand resources of the Atlantic inner continental shelf, Maine to Virginia}

\section{Workshop on Dredging, Beach Nourishment and Bird Conservation Atlantic Coast, Maine to Virginia}

\section{October 2005}

S. Jefiress Wriliams (iwilliams@usgs.gov)

U.S. Geological Suney

Woods Hole, MA

Slide 1. Title Image: Regional scale understanding of the geologic character and sand resources of the Atlantic inner continental shelf, Maine to Virginia. 


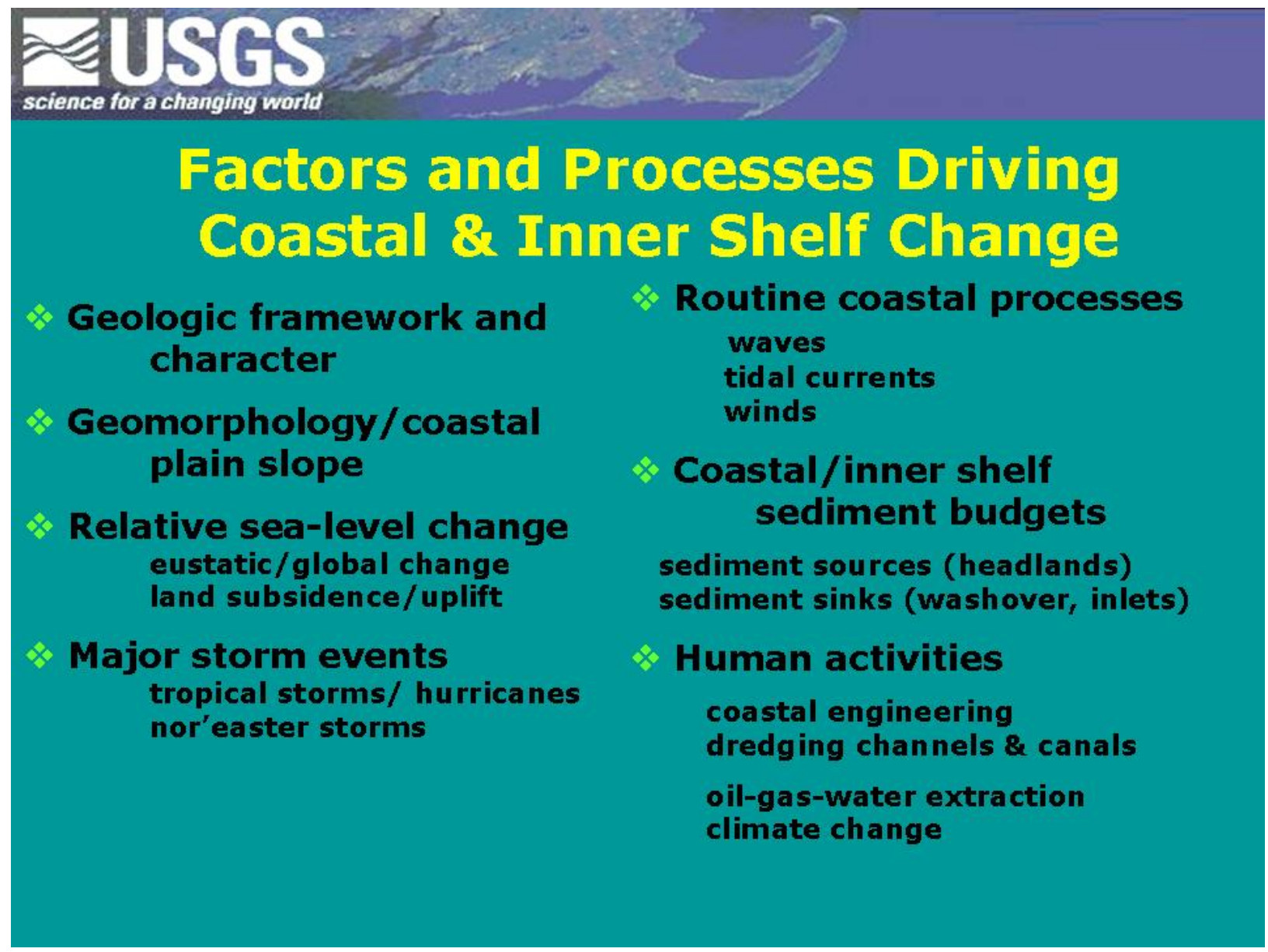

Slide 2. Coastal change is driven by many factors, including local geology, geomorphology, sealevel change and weather. 


\title{
Need for seafloor sediment maps and sand resource assessments
}

\begin{abstract}
$\leftarrow>70 \%$ of US coasts undergoing long-term chronic erosion.

* Coastal development, population and recreation continue to increase for all coastal regions.
\end{abstract}

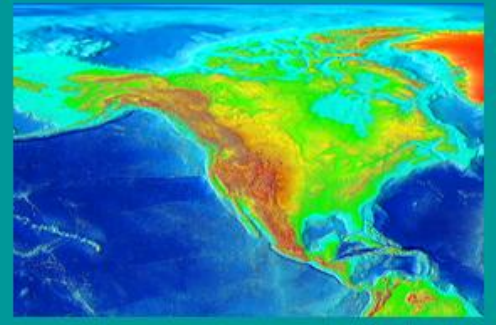

* Global climate change is likely to increase storminess and accelerate sea level rise, resulting in increased coastal vulnerability to erosion and flooding hazards.

* Beach nourishment is increasingly the preferred method of mitigating coastal erosion and restoring ecosystems.

* Large volumes of high quality sand are required for nourishment, on-land resources are limited, and marine sand bodies on inner shelf regions are increasingly attractive targets.

* The geologic character of marine sand bodies is highly variable resulting from dynamic marine transgression processes over the past $\sim 20 \mathrm{k}$ years.

SJW

Slide 3. Scientific knowledge and understanding of the sea floor composition and morphology is important in understanding coastal change. 


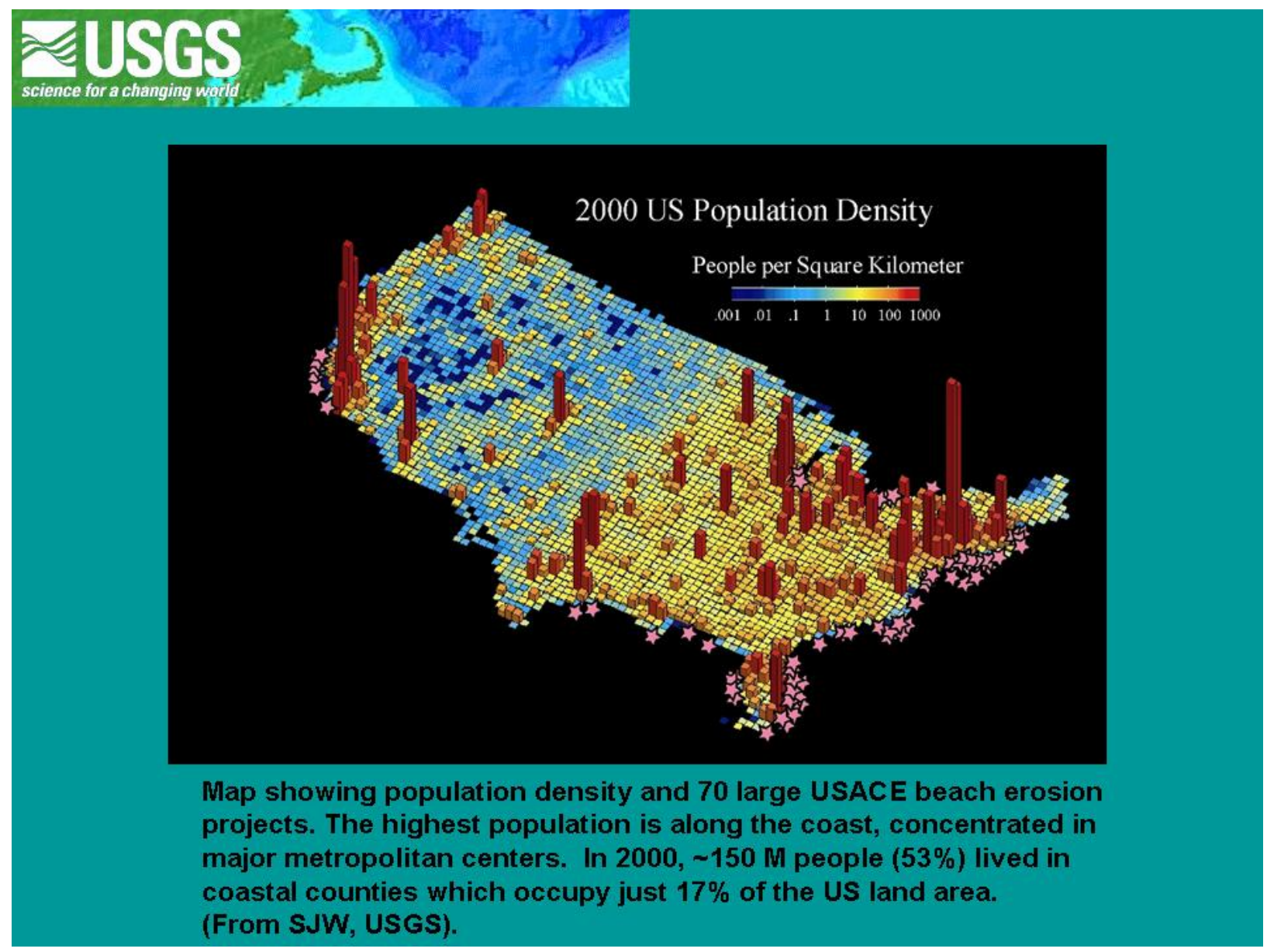

Slide 4. Population density map of the continental U.S., highlighting the proximity of large population and development in the coastal areas. 


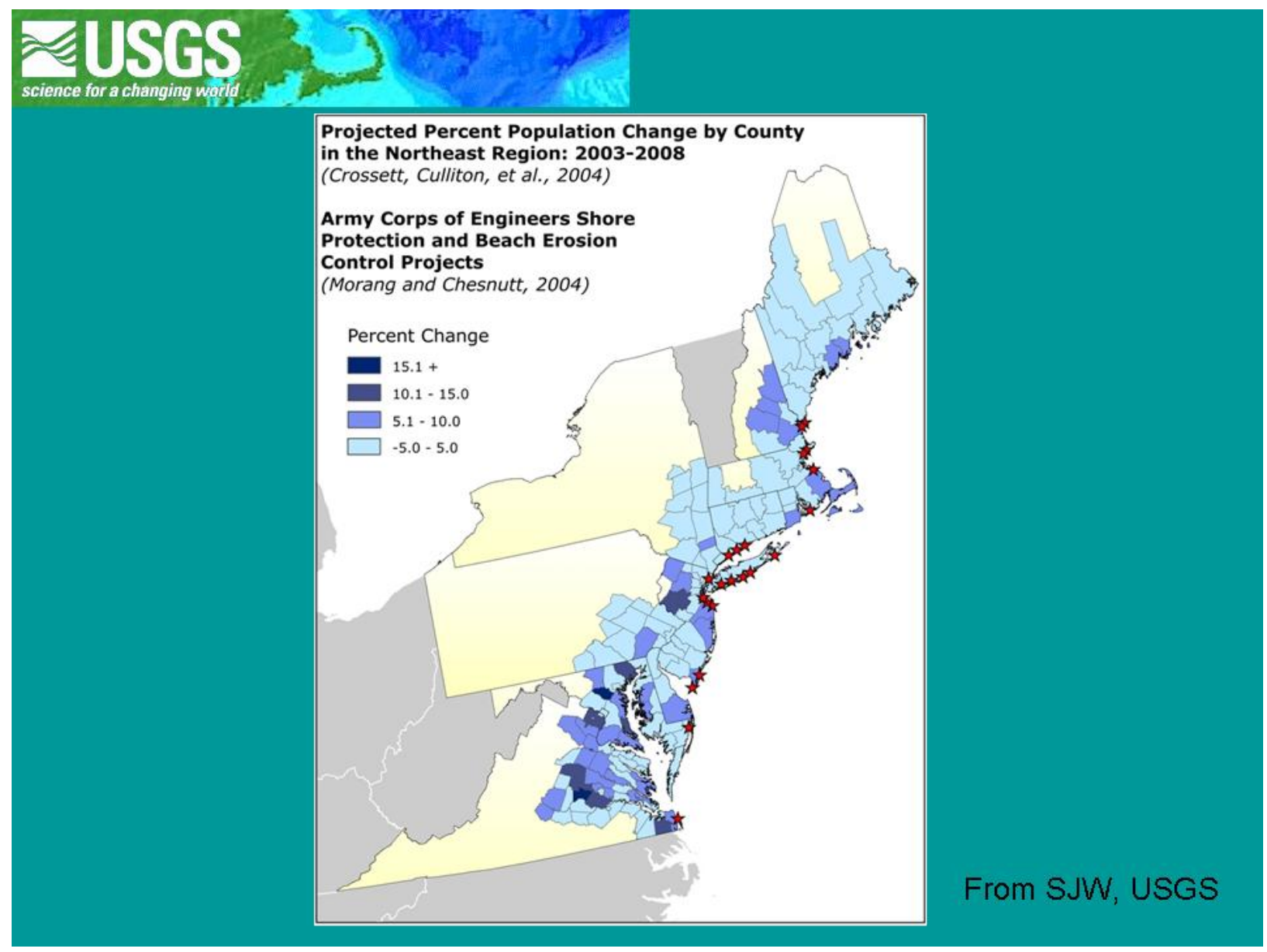

Slide 5. Map showing the projected increases in population for the Northeast region. 


\section{Motivation for sea-level rise research}

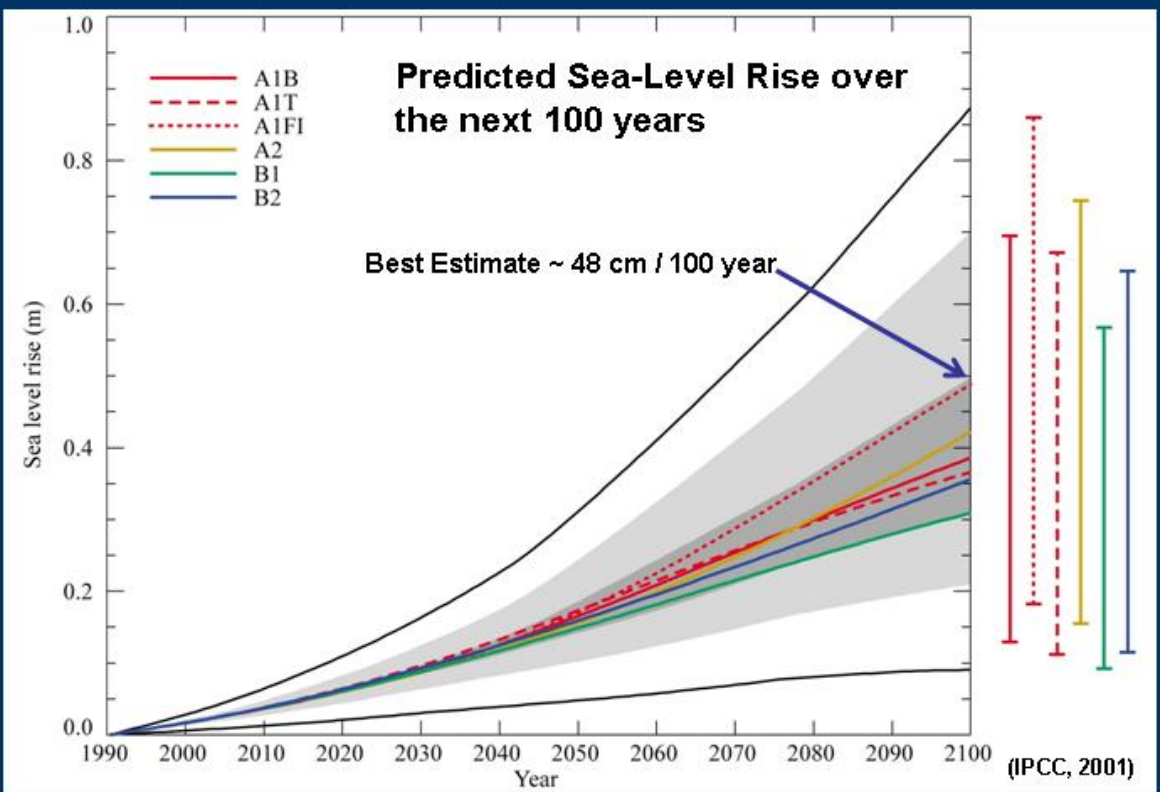

\section{\#USGS}

Slide 6. Chart of the projected sea-level rise for the next 100 years. 


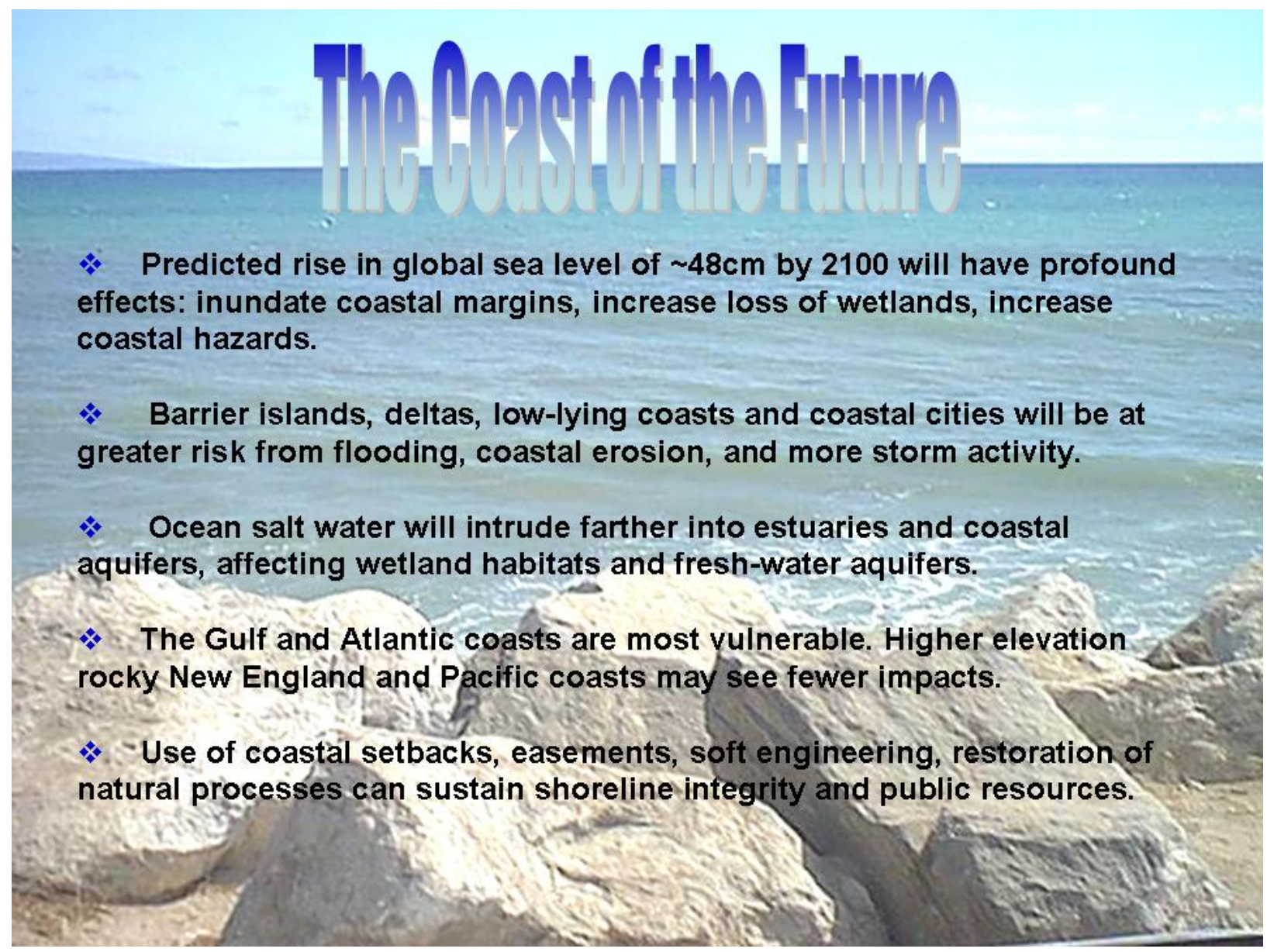

Slide 7. Sensitive coastal areas will feel the most dramatic effects of sea level rise in future decades. 


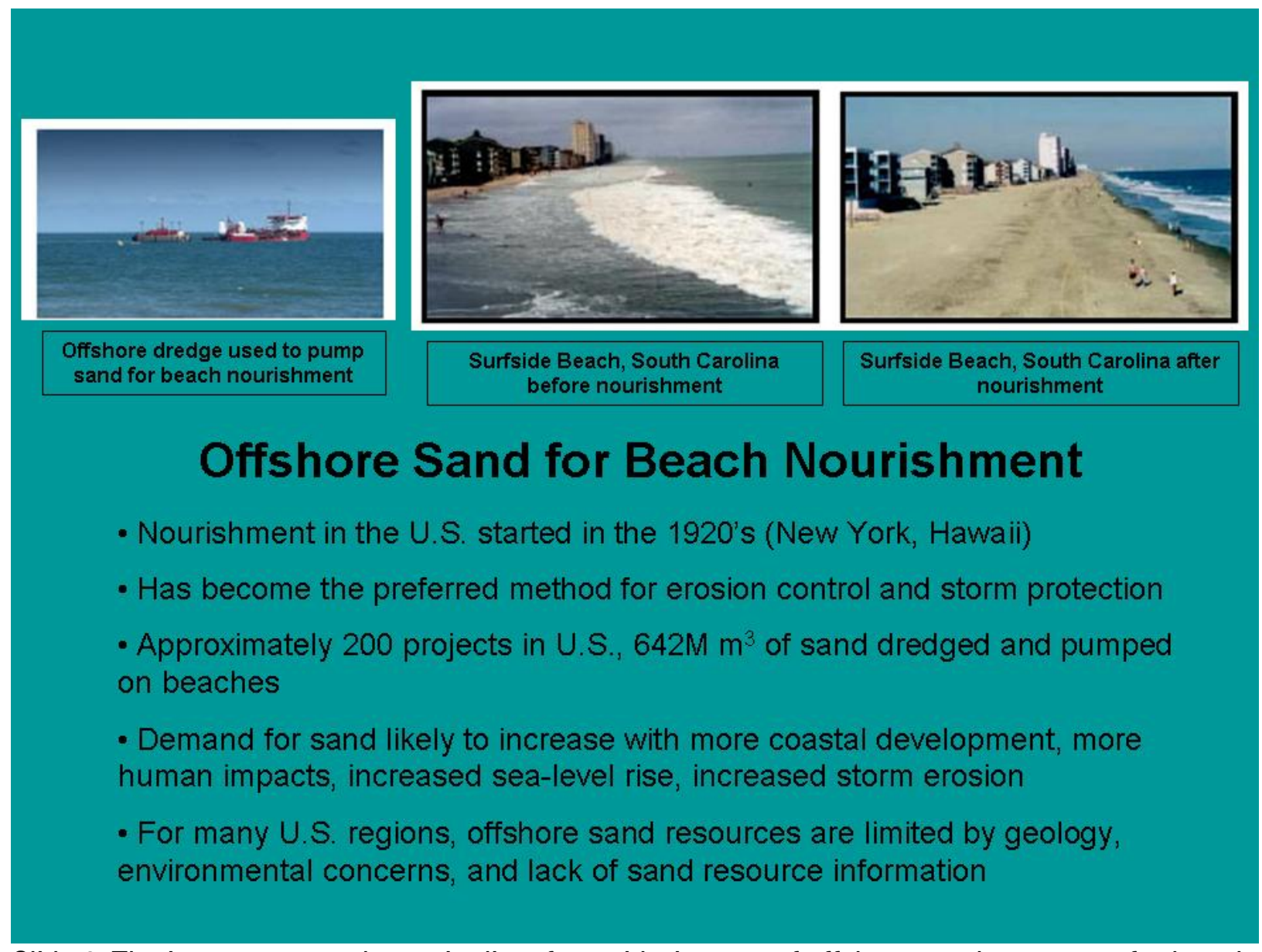

Slide 8. The importance and practicality of considering use of offshore sand resources for beach nourishment to mitigate erosion. 


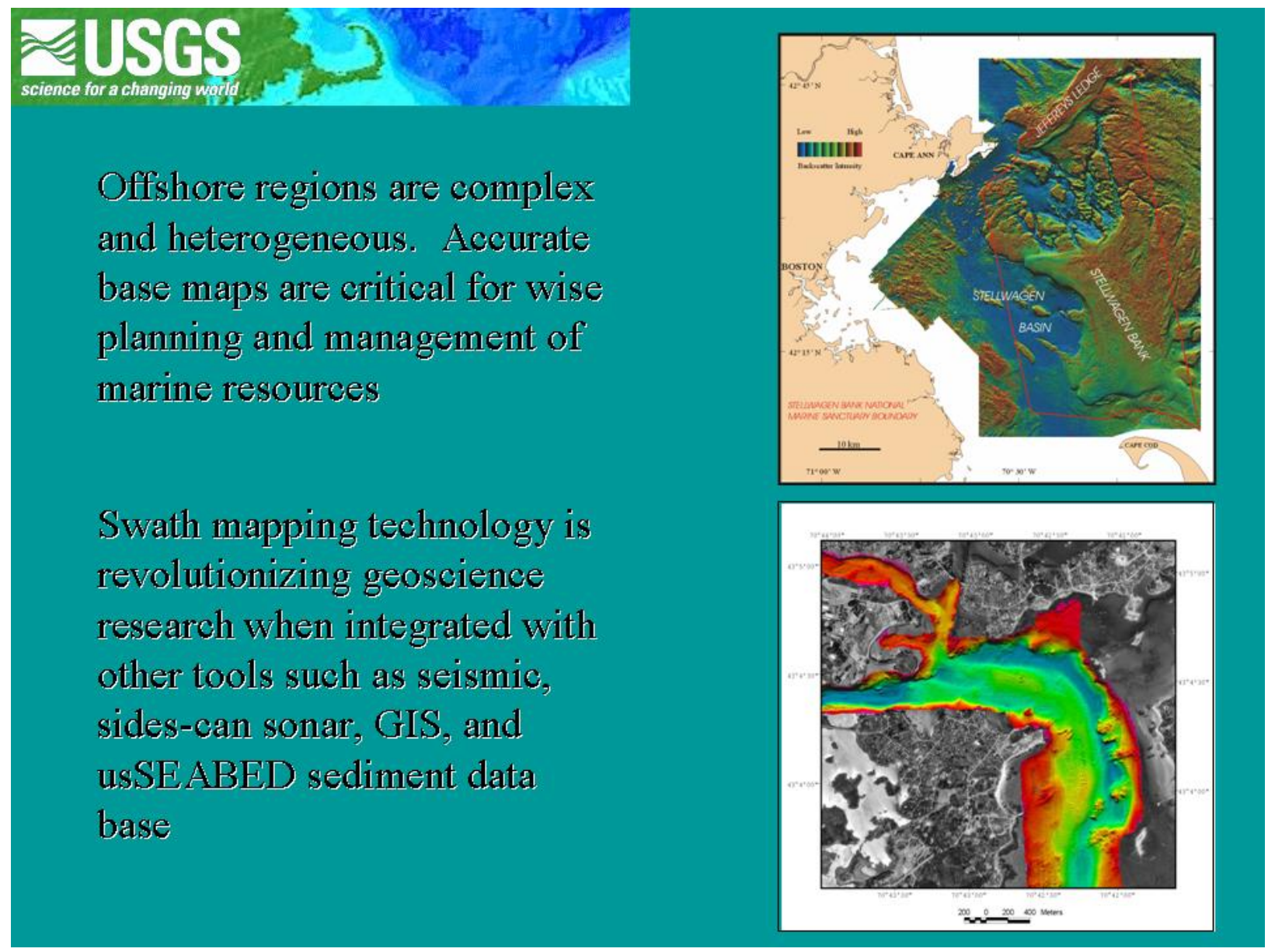

Slide 9. The heterogeneous nature of offshore deposits makes base maps essential tools in management and planning for coastal areas. 


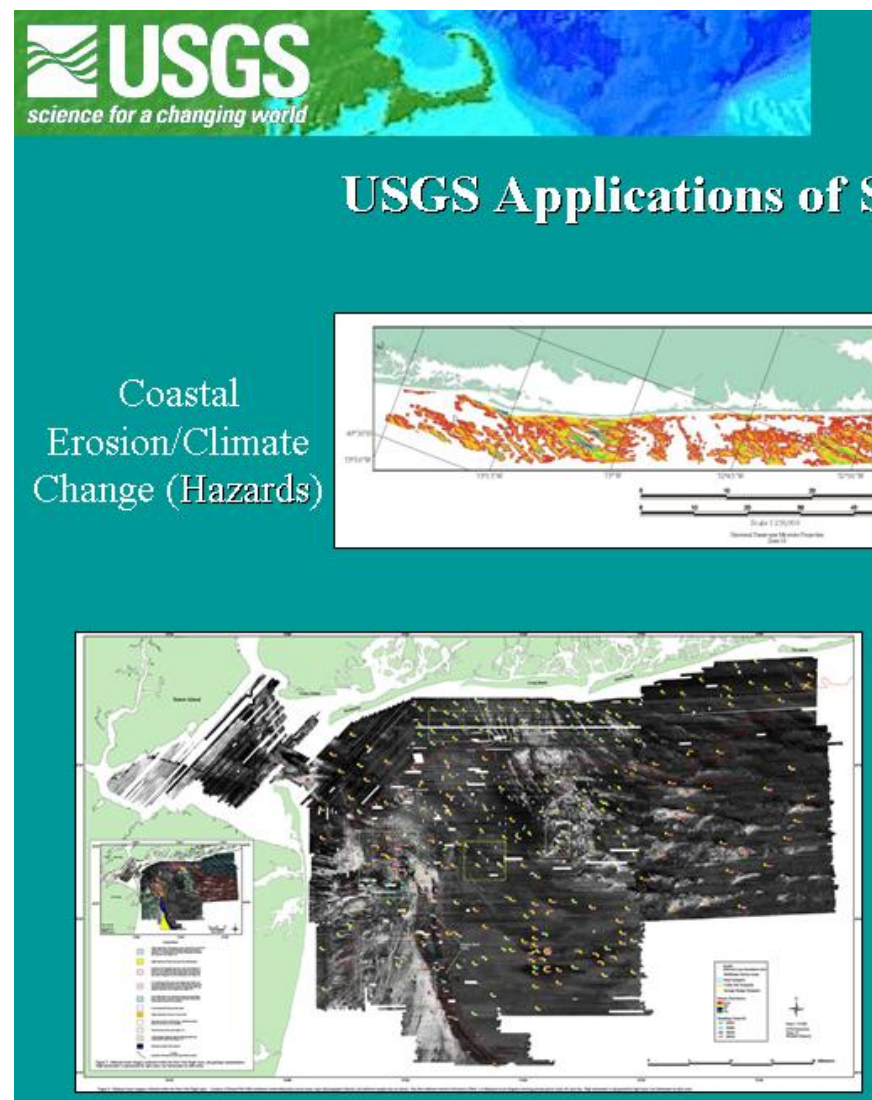

Nearshore

Sediment

Distribution and

Dynamics

(Resources)
Sediment Contamination (Environment)

Slide 10. Examples of surveys done by the USGS in sea floor mapping.

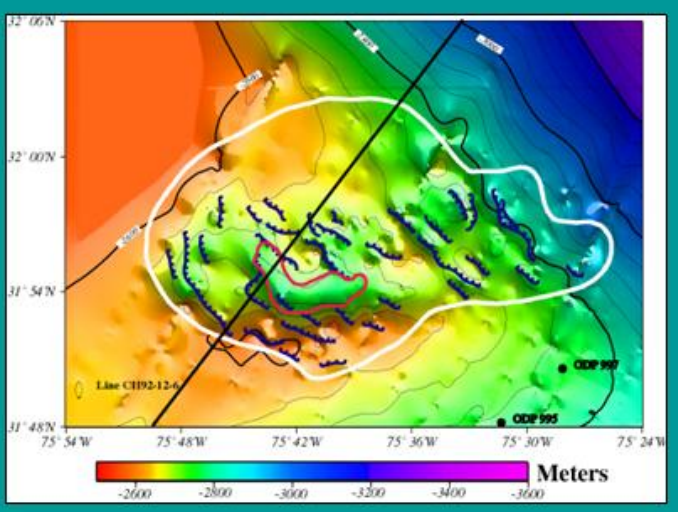

Gas Hydrates (Hazards/Resources) 


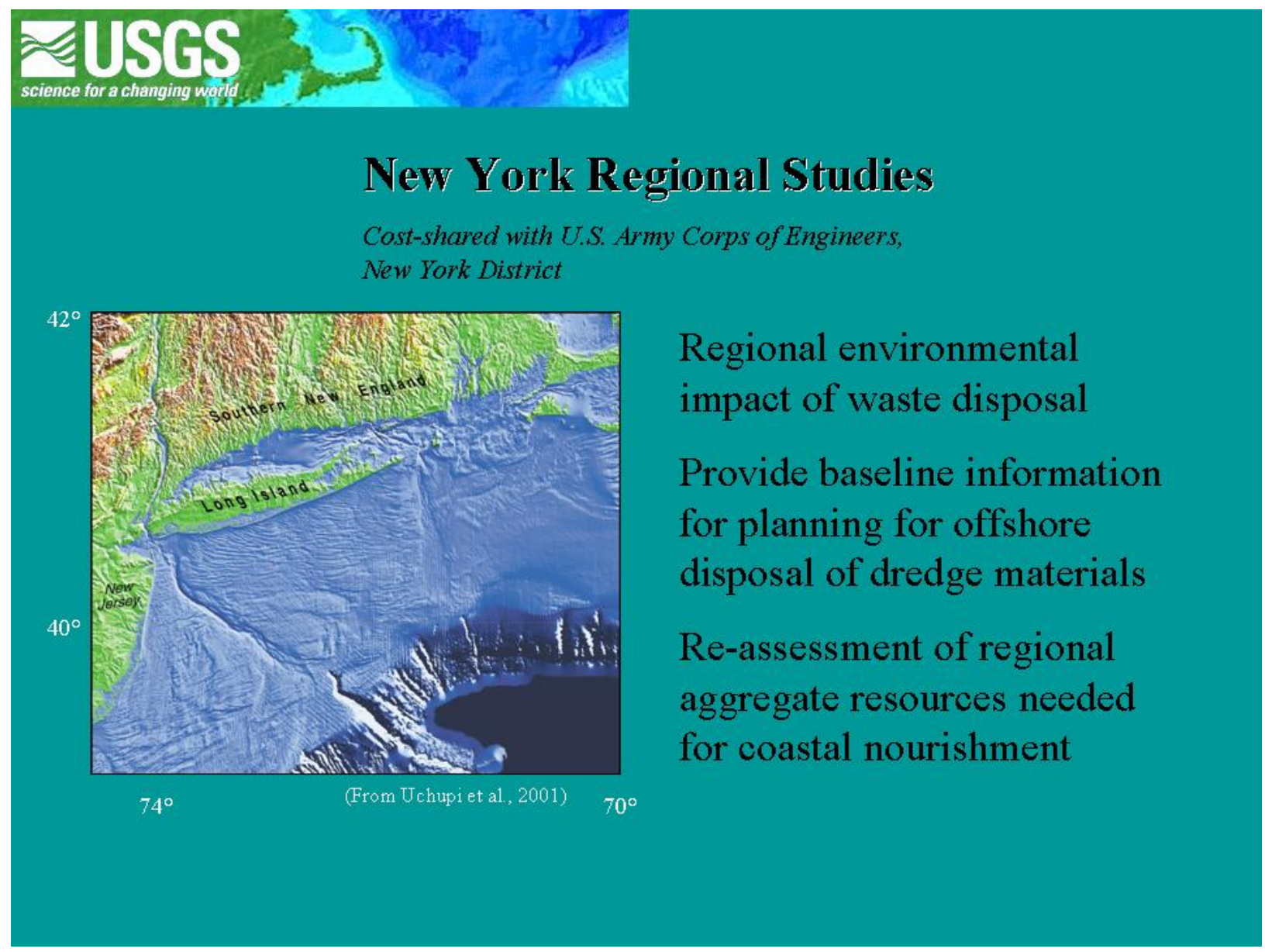

Slide 11. Regional studies by the USGS and Army Corps of Engineers in the New York Bight region. 


\section{ZUSGS}

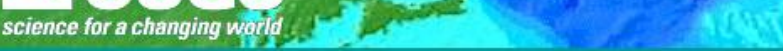

Study Area Was Mapped Using Side-scan Sonar, Multibeam Echosounder, Seismic, and Sediment Sampling Techniques
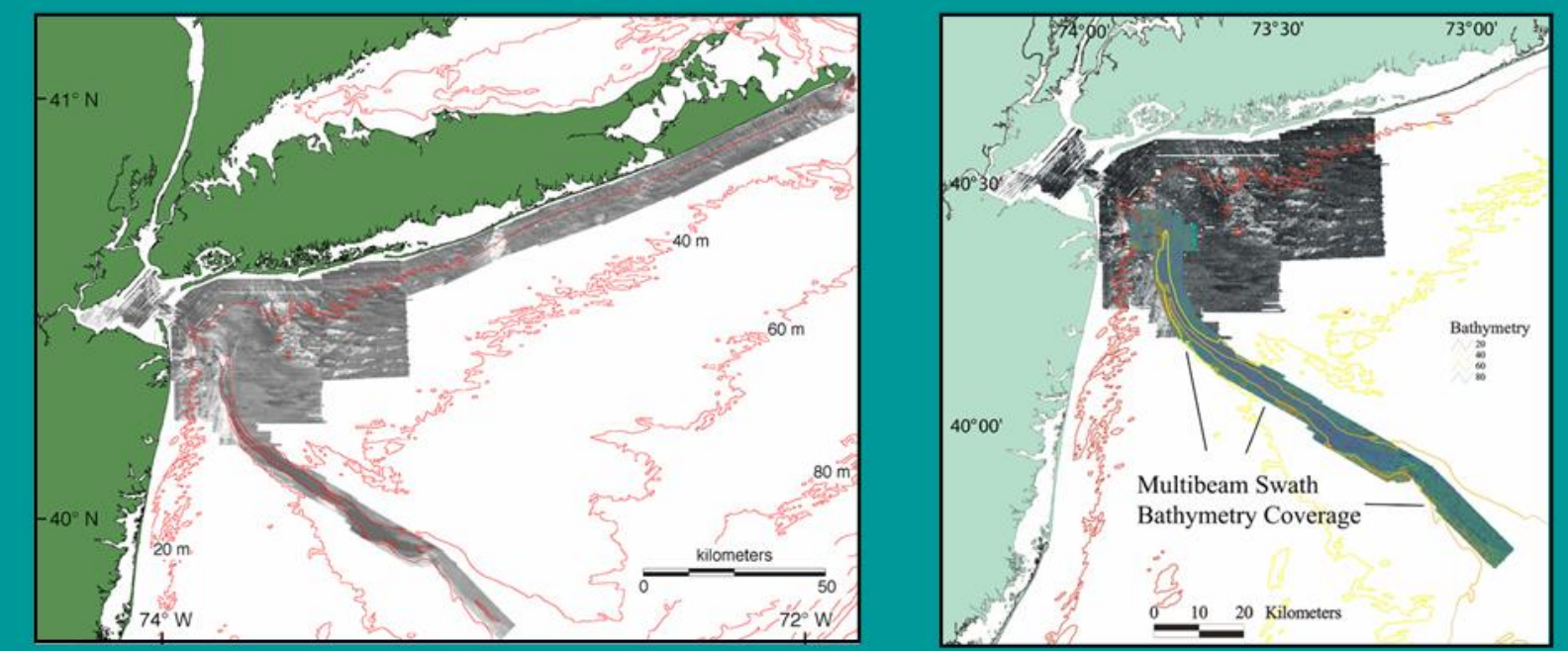

Slide 12. Multidisiplinary studies of New York Bight and coastal Long Island. 


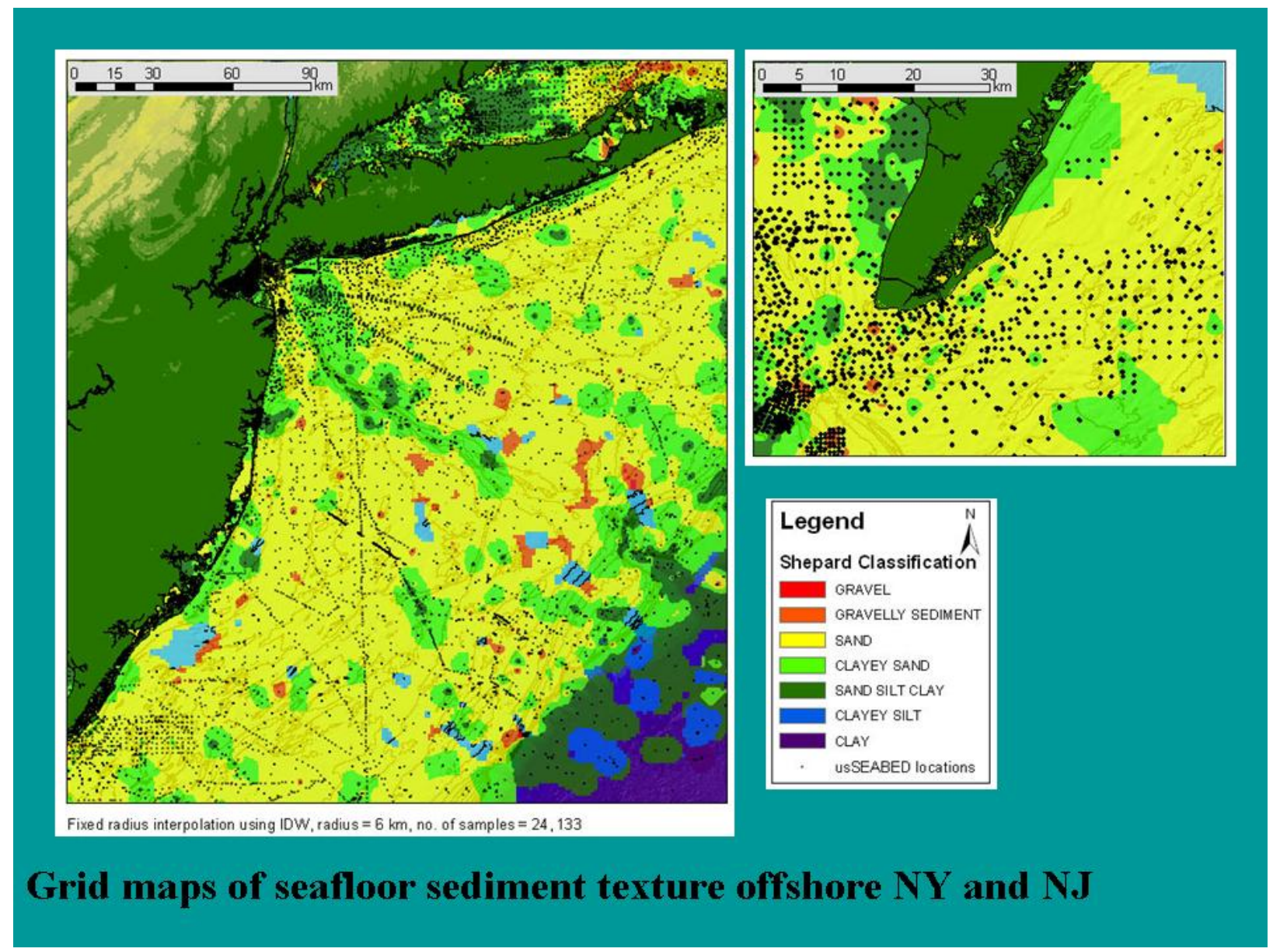

Slide 13. Sea-floor sediment texture maps produced using usSEABED data. 


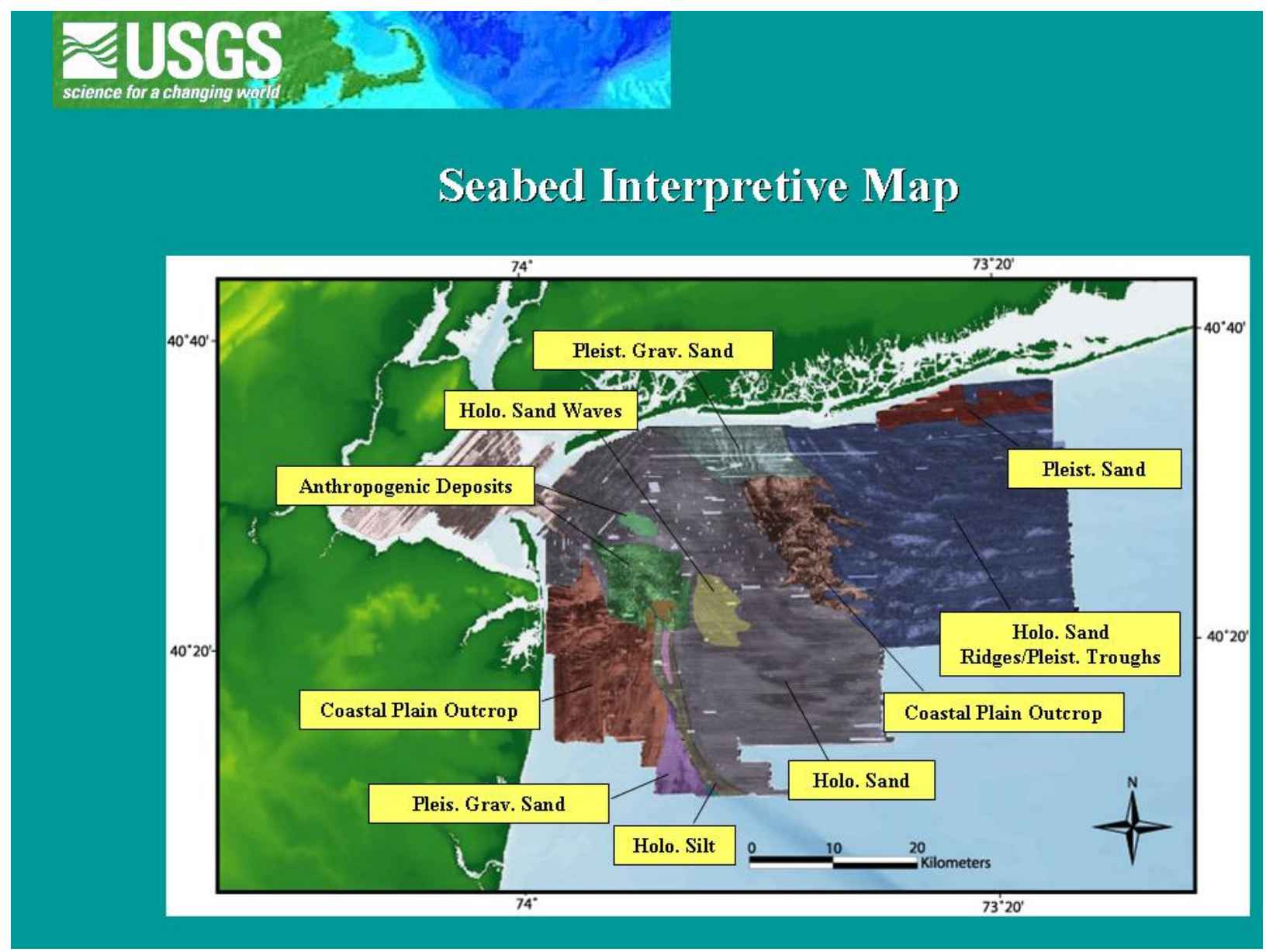

Slide 14. Interpretive map of sea-floor composition in New York Bight. 


\section{ZUSGS \\ science for a changing world}

Distribution of Holocene Sediment:

Sediment Distribution Defines Paleo-Barrier-Island System

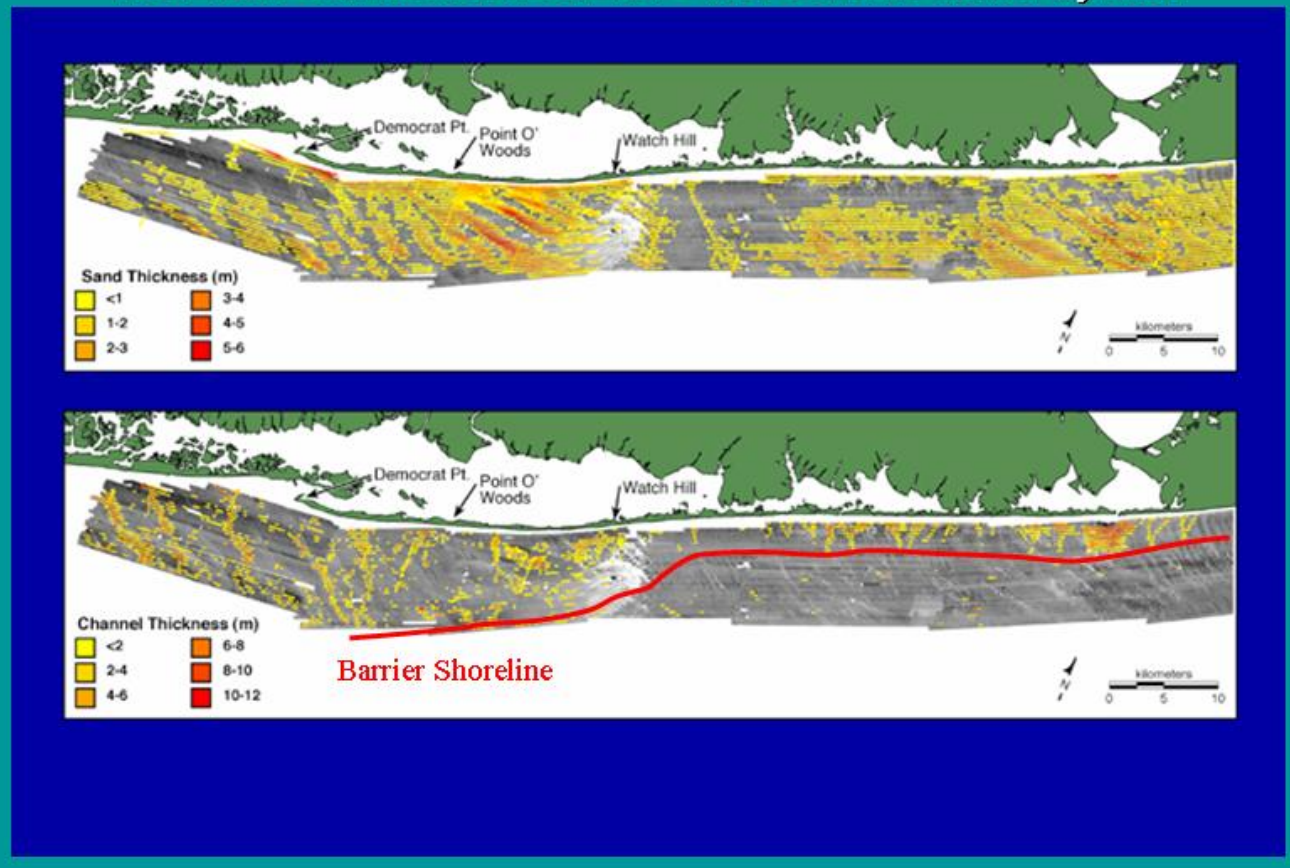

Slide 15. Distribution of Holocene sediments along southern Long Island, and paleobarrier island system. 


\section{ZUSES}

science for a changing world

\section{Marine Aggregate Resources and Processes}

\section{Project rationale and directions-}

A direct response to Science for Decision Making, NRC (1999), Grand Challenge 1, Establish the geologic framework of US coastal \&

marine regions....need to integrate existing info into national assessments... the distribution and quality of marine mineral resources.

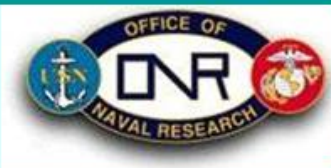

Federal (MMS, USACE, NOAA, EPA), coastal state and local regulatory agencies need marine science information for managing coastal resources, planning and decision making.

The USGS is the principal federal science mapping agency for interpreting and describing the geologic framework and character of US continental margins and for providing information on availability of mineral resources within the EEZ.

Project, in collaboration with others, is carrying out a series of regionalscale studies of the shelf sedimentary character and associated sand and gravel resources.

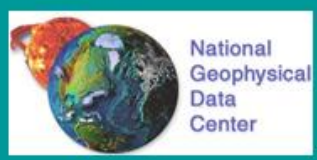

The regional studies comprise a suite of reports, maps, data bases of seafloor sediment character, resulting in an integrated national assessment of potential offshore sand and gravel resources.

Reports, maps and assessments provide information and guidance to federal and state agencies having resource and seafloor regulatory and management responsibilities.

UNO $\frac{\text { COASTAL RESEARCH LABORATORY }}{\text { Department of Geology end Geophysics }}$

Slide 16. The USGS Marine Aggregate Resources and Processes rationale and focus. 


\section{Marine Aggregate Resources and Processes}

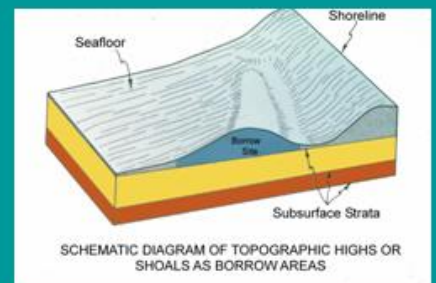

SHOALS AS BORROWAREAS
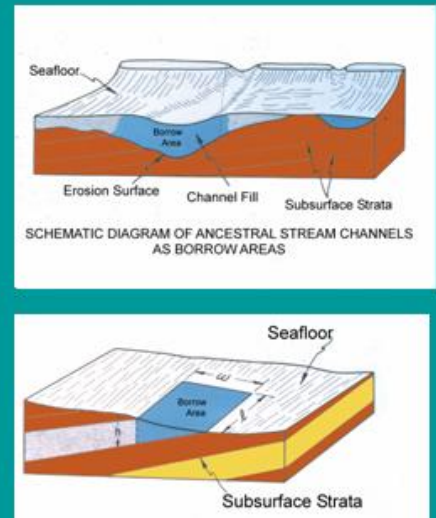

SCHEMATIC DIAGRAM OF COASTAL PLAIN SURFACE EXPOSURES AS BORROWAREAS

\author{
A Parmership of USGS, ONR, NOAA, MMS, USACE, States \\ and Academia to Characterise the Nation's Segfloor \\ Sedimentay Character and Assess Aggregate Resources
}

Themes

- Scientific evaluation and synthesis of available maps and reports (published, gray literature, files)

- Compilation and integration of legacy marine geologic data into usSEABED database system

- Scientific interpretation of shelf history and processes, marine sand body origins and evolution

- Reports, GIS digital map products and usSEABED data available on internet and in publications

Slide 17. The USGS Marine Aggregate Resources and Processes scientific and data product goals. 


\section{Marine Aggregate Resources and Processes}

Project Tasks

* Regional assessment for New York \& New Jersey

* Regional assessment for Louisiana

* Regional assessment for Gulf of Maine

* Development of usSEABED as a national GIS system for seafloor sedimentary character

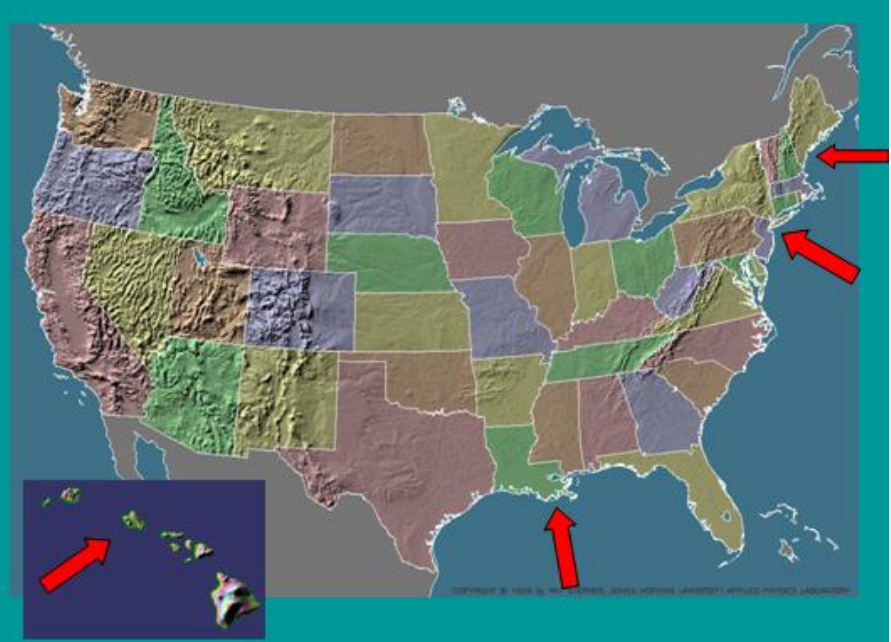

Slide 18. The USGS Marine Aggregate Resources and Processes projects and targeted geographic regions for assessments. 


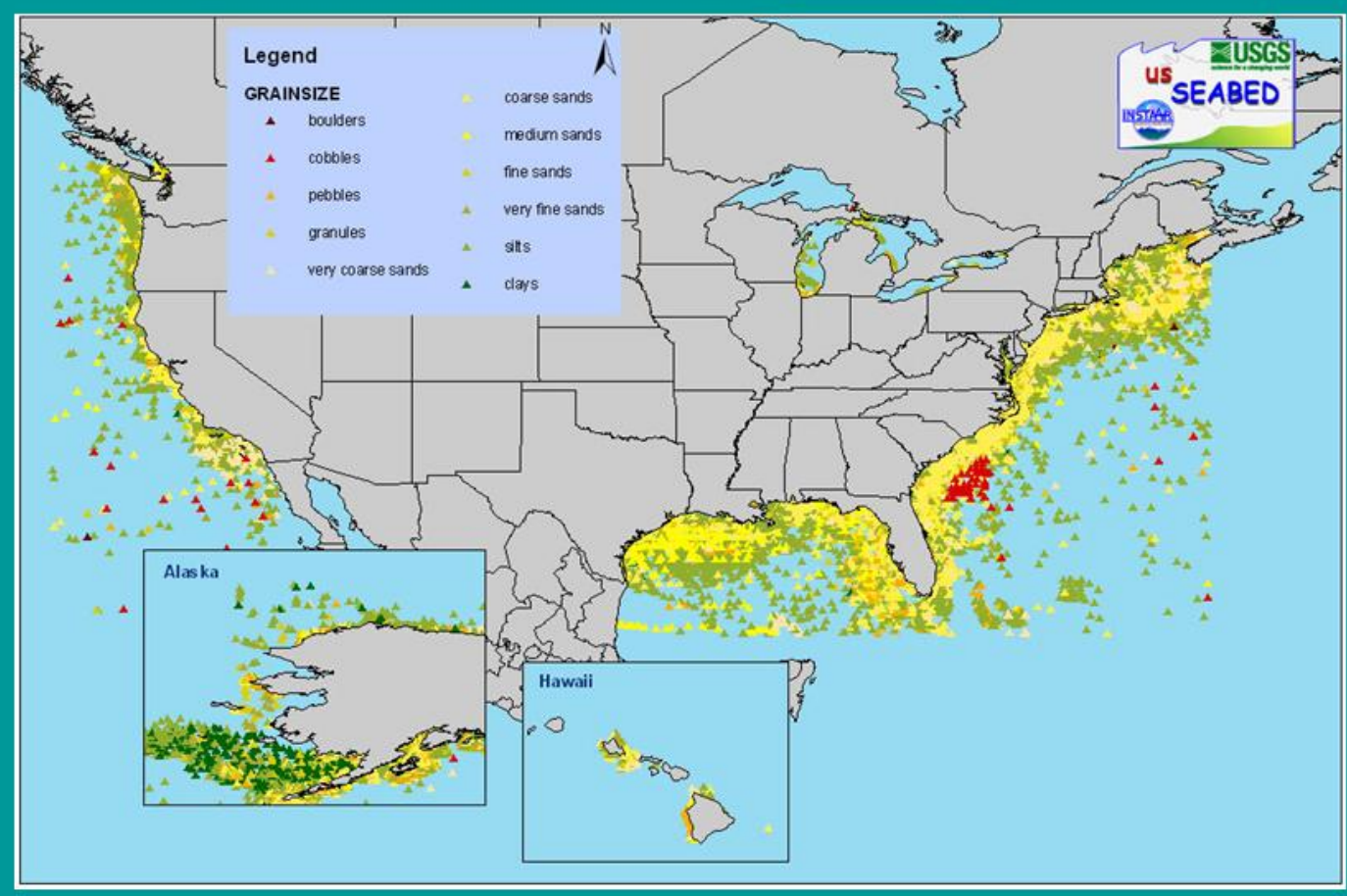

Map of usSEABED data coverage in US EEZ showing seafloor sediment texture

Slide 19. The usSEABED data coverage within the U.S. Exclusive Economic Zone of the continental United States. 


\section{Summary}

Shorelines and coastal development will be even more vulnerable to hazards in the future. Need for

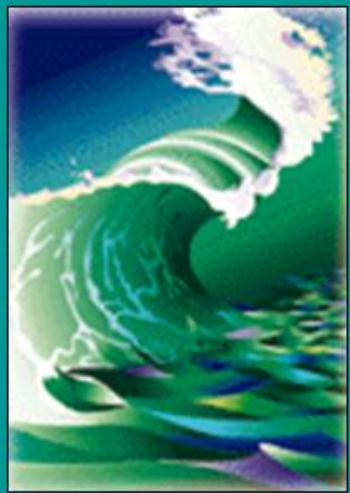
offshore sand for nourishment will increase. Sand volumes for sustainable shore protection are uncertain for many regions.

Geologic framework influences shelf sediment character, distribution, and shelf physiography.

Sediment processes acting on the inner shelf influence the evolution of the shelf and adjacent coast.

Better understanding of the shelf geology can aid our ability to predict future coastal change and to plan for sustainable use of coastal regions.

Slide 20. Summary of coastal processes and the USGS Marine Aggregate Resources and Processes contribution to better understanding of these processes. 


\section{us SEABED : Offshore Surficial Sediment Data Releases}

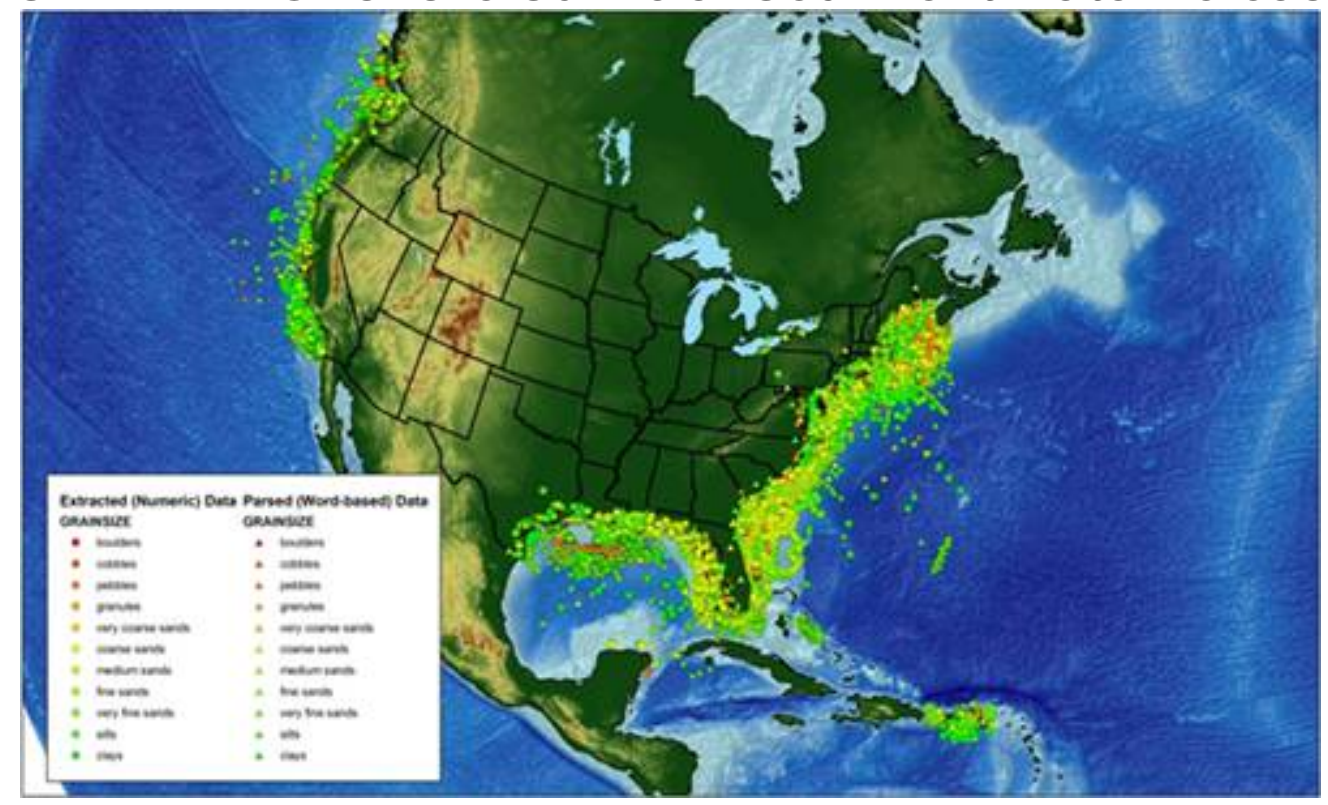

\section{An Overview of usSEABED}

The value of seafloor sedimentary data is becoming increasingly more important with recent prevalence in bathymetric mapping (i.e. the need for ground truthing), utlities planning (e.g., offshore wind and tidal turbines), and homeland security measures in place around America 's extensive port system. This has elevated the importance of historical data sets of seafloor character as new data is not only expensive, but also time consuming to collect.

These reports provide a synthesis compilation of published and unpublished sediment texture and other geologic data about the seafloor from a diverse range of sources. Each report describes the usSEABED database and the dbSEABED system, developed to bring assorted data together in a unified database to provide examples of maps displaying attributes such as grain size and sediment color. This database contains information that is the scientific foundation for the USGS's Marine Aggregate Resources and Processes Assessment and Benthic Habitats projects, and is already proving useful to the marine science community at large.

The usSEABED database holds data for the entire US EEZ and is an ongoing project of the USGS Coastal and Marine Geology Program of Santa Cruz, CA; Woods Hole, MA; and St. Petersberg, FL; and the University of Colorado. We expect to release new Data Series as significant new data are included in the database. 
usSEABED

The usSEABED database, which covers the U.S. EEZ, is built using the dbSEABED processing software created at the University of Sydney, Australia, and the University of Colorado . It has companion databases built along similar lines: auSEABED for Australia , balticSEABED and a global database, goSEABED. Each of these databases rely on pre-existing data, both published and unpublished, from a variety of sources (such as federal, state, regional, and local agencies and consortiums, as well as research institutions) to mine and extrapolate useful data about the seabed.

The dbSEABED program allows source reports to be compiled in a standardized format, and extrapolates information across a series of data types and equipment, such as physical sampling equipment (sounding, grabs, and cores) or virtual sampling (photographs, videos, geophysics). These data may be numeric lab- or probe- based textural, acoustic, geochemical, and geophysical data and/or verbal (linguistic) descriptions of grabs, cores, or photographs, or a combination of any of these.

The usSEABED database, using the dbSEABED program, differs from other US databases in that it incorporates both numerical and linguistic data on sediment texture, biology, seafloor characteristics such as hardness or sediment ripples, acoustic properties, and geochemical and geotechnical analyses. In the usSEABED database, most data held in reports are mined and extended for additional information that increases the data density over the seabed, allowing for more complete maps and information.

\section{Data Catalog and GIS Layers}

The data supplied on these publications is made available with geographic coordinates to allow the data to be incorporated into a Geographic Information System (GIS). Federal Geographic Data Committee (FGDC) metadata are included with data layers in three formats: HTML, FAQ, and text. Layers include those for the Extracted (EXT), Parsed (PRS), Calculated (CLC), Components (CMP) and Facies (FAC) output files along with basemap layers such as coastlines and EEZ and state boundaries compiled together in an ArcView TM project file.

\section{Atlantic, Pacific and Gulf Coast Publications}

The publications are broken down into geographical subsets for the entire Exclusive Economic Zone (EEZ) of the United States . Publications of the Atlantic coast, the Gulf Coast and the Pacific ( California, Oregon, Washington) Coast are available currently. Companion publications for Alaska and Hawaii are also expected. The following maps show the distribution of Parsed (PRS) and Extracted (EXT) data for each Data Series publication. The PRS outputs are based on descriptive (word-based) data through the application of Fuzzy Set Theory. The EXT outputs are based on numeric data extracted from the data resource files through data mining.

\section{Frequently Asked Questions}

The publications provide answers to many important Frequently Asked Questions (FAQs) such as:

- How does dbSEABED make word data con formable with numeric data?

- What is Fuzzy Logic and how does it work? 
- What is involved in importing datasets into dbSEABED?

- What if a user doesn't want to use the word-based data for creating maps?

- What quality control measures are in place for dbSEABED?

- How does word-based descriptive data relate to numeric-value analytical data?

\section{For More Information}

We appreciate feedback on usSEABED, both in usefulness and in error detection. Please contact us with issues, questions and/or data to contribute to the growing usSEABED information system in the U.S. EEZ.

http://walrus. wr.usgs. gov/usseabed/index.html

Matt Arsenault: for adding Atlantic Coast and/or Gulf Coast data

U.S. Geological Survey Woods Hole Science Center 384 Woods Hole Road Woods Hole, MA 02543-1598 Tel: 508-548-8700 Email: marsenault@usgs.gov

J ane Reid: for information about the Benthic Habitats project and/or adding Pacific Coast, Alaska or Hawaii data

U.S. Geological Survey Pacific Science Center 400 Natural Bridges Drive Santa Cruz, CA 95060 Tel: 831-427-4727 Email: jareid@usgs.gov

J eff Williams: for information about the Marine Aggregates project

U.S. Geological Survey Woods Hole Science Center 384 Woods Hole Road Woods Hole , MA 02543-1598 Tel: 508-548-8700 Email:jwilliams@usgs.gov

Chris Jenkins: for information and answering questions about the dbSEABED program as well as adding global data

University of Colorado Institute of Arctic and Alpine Research 1560 30th Street Campus Box 450 Boulder CO, 80309-0450 Tel: 303-735-5250 Email: chris.jenkins@ colorado.edu Larry Poppe: for answering questions about sediment data collection; laboratory and analysis techniques

U.S. Geological Survey Woods Hole Science Center 384 Woods Hole Road Woods Hole, MA 02543-1598 Tel: 508-548-8700 Email: lpoppe@ usgs.gov

\section{Disclaimers}

\section{General}

This CD-ROM publication was prepared by an agency of the United States Government. Neither the United States Government nor any agency thereof, nor any of their employees, makes any warranty, expressed or implied, or assumes any legal liability or responsibility for the accuracy, completeness, or usefulness of any information, apparatus, product, or process disclosed in this report, or represents that its use would not infringe privately owned rights. Reference herein to any specific commercial product, process, or service by trade name, trademark, manufacturer, or otherwise does not necessarily constitute or imply its endorsement, 
recommendation, or favoring by the United States Government or any agency thereof. Any views and opinions of authors expressed herein do no necessarily state or reflect those of the United States Government or any agency thereof. Although all data published on this CD-ROM have been used by the USGS, no warranty, expressed or implied, is made by the USGS as to the accuracy of the data and related materials and/or the functioning of the software. The act of distribution shall not constitute any such warranty, and no responsibility is assumed by the USGS in the use of these data, software, or related materials.

\section{Accessibility}

Section 508 and Accessibility at the USGS

The USGS is committed to and is making every possible effort to ensure that all electronic and information technology developed, procured, maintained, or used by the USGS is accessible to people with disabilities, including both employees and the customers we serve. If you cannot fully access the information on this CD-ROM, please e-mail the contact person or group named in the contact information provided on this CD-ROM, and they will help you. This may include providing the information to you in an alternate format.

For further information and to learn more about the regulations governing the accessibility of Federal electronic information products, visit the Section 508 Web pages listed below:

- Section 508 home page - www.section508.gov/

- Access Board - www.access-board.gov/508.htm

- Web Accessibility Initiative (WAI) - www.w3.org/WAI/

\section{README}

\section{Background}

This report contains a compilation of available textural data and numeric data based on lithologic descriptions generated from surficial sediment samples from offshore the New York and New Jersey coast of the United States. These data are a subset of the usSEABED data for the Atlantic coast as described in Reid and others (2005). More than 340,500 samples containing sediment grain size and lithology have been compiled as part of the U.S. Geological Survey's Marine Aggregate Resources and Processes and National Benthic Habitats projects. These data are being used to update the current maps on surficial sediment distribution for the New York Bight region.

Many of these data layers were compiled using gray literature or unpublished sources and have not been available in digital form prior to publication of USGS DS-118. These data have been converted to Environmental Systems Research Institute, Inc. (ESRI) "shapefile" format for use in the project Geographic Information System (GIS), and data are supplied with complete FGDC compliant metadata. Sediment data and sample identifiers are also supplied in flat-file format and Microsoft Excel spreadsheet files for those users who may not have GIS access. 


\section{CD-ROM Contents}

There are three top-level files and three top-level directories contained on this CD-ROM. The top-level files are:

- readme.txt (the ASCII version of this file) - This file contains a description of this CDROM and may be viewed or printed by the user with any system program capable of opening an ASCII text file.

- index.htm - This file is intended to be the starting point for CD-ROM access. It is written in the Hyper-Text Markup Language utilized by the World Wide Web (WWW) project and must be opened with a WWW browser. Once opened, the user may browse the CD-ROM's contents as they would browse pages from the WWW.

- nynj.apr - An ArcView 3.3 project file containing the GIS data layers. This file facilitates the viewing of the shapefiles and related image data sets. If the user does not have ArcView available, they may access the shapefiles by downloading ESRI's ArcExplorer software at no charge from the ESRI Web site

The three main directories include:

Directory htmldocs/

- This directory contains five directories and sub-directories used to format the HTML files and supporting graphics to allow the user access to the report via any World Wide Web browser. The major sub-directories/files include the following:

- NYB.css/ - this file is the cascading style sheet used to format the layout of the HTML pages

- files/ - this directory contains files offered in the various HTML pages

- images/ - this directory contains images used in the HTML page layouts

- Directory data/

- This directory contains four subdirectories that contain the various GIS data layers and appropriate metadata. The sub-directories including the following data:

- basemaps/ - basemap data used in the ArcView project file. Basemap data include a general US land and coastline shapefile, U.S. Exclusive Economic Zone and the 3 nautical mile state and Federal boundary.

- bathymetry/ - this directory contains the bathymetric file for the New York-New Jersey study region.

- stations/ - this directory contains information on available sea floor images from the New York-New Jersey region.

\section{Downloading Files and Images}

\section{Downloading a selected file with Microsoft Internet Explorer}

To download a data file while using Microsoft Internet Explorer, the user should select the desired file by placing the system pointer on the file name from the data list and pressing the right mouse button. This will display a pull-down menu from which the user should drag the mouse to highlight "Save target as ..." and release the mouse button. Internet explorer will then prompt the user to specify the name and location of where the selected data file should be saved. 


\section{Downloading a selected file with Netscape}

To download a data file while using the Netscape browser, the user should select the desired file by placing the system pointer on the file name from the data list and pressing and holding the right mouse button. This will display a pull-down menu from Netscape. The user should drag the mouse to highlight "Save this link as ..." and release the mouse button. Netscape will then prompt the user to specify the name and location of where the selected data file should be saved. Different operating systems and newer versions of Netscape might work somewhat differently.

\section{Technical Notes}

This disk has been tested for use on computers having Windows 98/NT/2000/XP and Macintosh operating systems.

Access to the data and information contained in this report was developed using the HyperText Markup Language (HTML) utilized by the World Wide Web (WWW). This allows the user to access the information using WWW information browsers (i.e., Microsoft Internet Explorer, Netscape). To start, open the file 'index.htm' at the top-level directory of this disc with your browser.

There are Internet links to USGS collaborators and Web sites included in this report. These links are only accessible if access to the Internet is available when browsing the CDROM, and if those linked sites are operating. 